MAGAZIN FÜR STUDIERENDE UND SOZIOLOGIEINTERESSIERTE

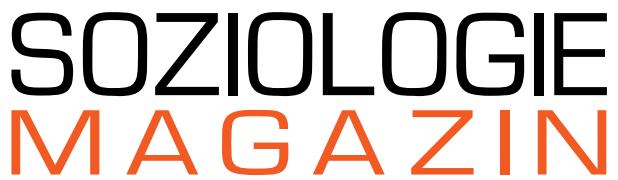

Publizieren statt archivieren

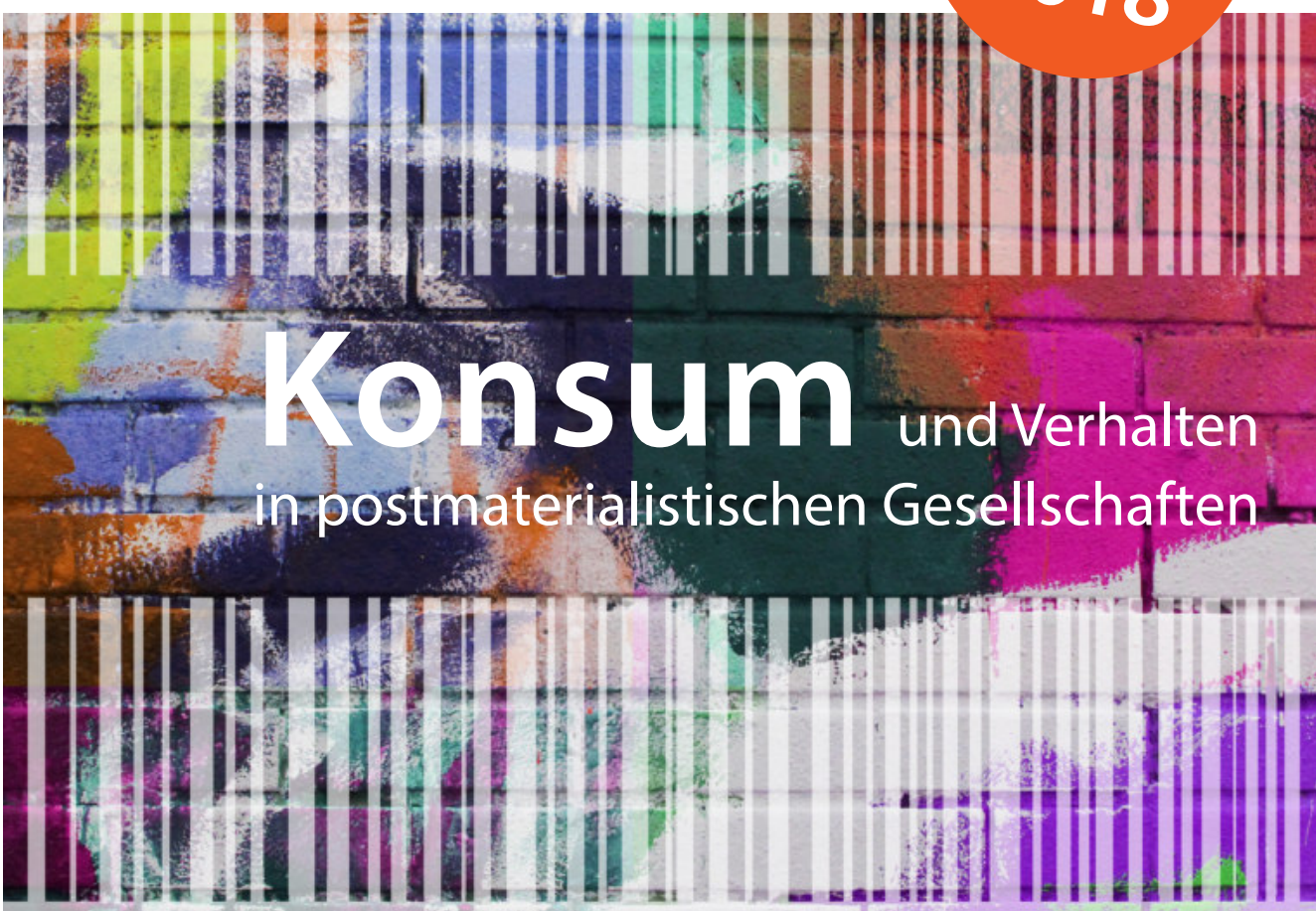

Konsumierend schreiten wir voran? Jedrzej(Yen) sulmowski Führe mich sanft ans gleißend helle Licht Enrico Hoerster| Eine Opposition gegen Autoritätssysteme des Marktes? soonim Shin

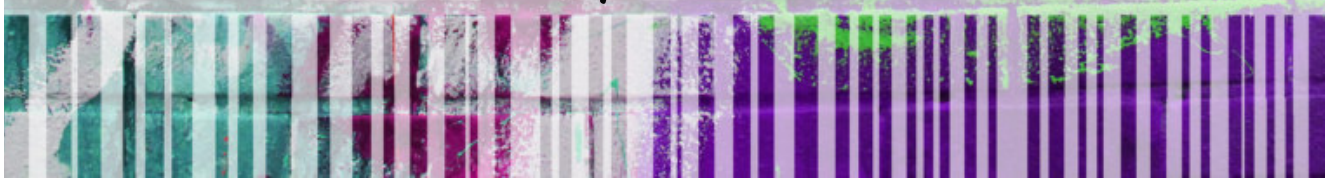

Außerdem: Expert_inneninterview zum Titelthema | Shopdropping von Mara Mürlebach | Die gemeinschaftsbildende Funktion des Konsumierens von Valerie Scheibenpflug | Call4Papers Ausgewählte Fachliteratur | Konferenzen- und Tagungstermine 2018/19 


\section{SOZIOLOGIE \\ MAGAZIN}

Publizieren statt archivieren

\section{Mach mit!}

Schick uns deine Fotografien!

Das SOZIOLOGIEMAGAZIN erscheint zweimal im Jahr jeweils zu einem bestimmten Thema und beinhaltet soziologisch relevante Beiträge, sorgfältig lektoriert und von einem Wissenschaftsbeirat fachlich begutachtet, Interviews, Buchreviews, Termine u.v.a.m. Parallel dazu gibt es im Internet den Wissenschaftsblog des Soziologiemagazins, um Diskussionen anzuregen und auf aktuelle Anlässe reagieren zu können.

Im Frühling 2019 erscheint unsere Ausgabe zum Thema:

\section{Rausch und Ekstase}

\section{Erkundungen der Spaßgesellschaft}

Hierzu möchten wir wieder zum Thema passende Fotos und deren Macher_innen im Magazin präsentieren. Selbstverständlich steht eine kurze Personendarstellung inklusive Kontaktdaten am Beginn jeder Veröffentlichung!
Die Fotografien sowie das Fotograf_ innenportrait erscheinen in unserem E-Journal und in unserer gedruckten Ausgabe.

Die Bildrechte bleiben selbstverständlich bei dem Fotografen/der Fotografin.

\section{Und so geht's:}

Wer bei uns veröffentlichen möchte, sendet einfach eine Vorauswahl von maximal fünf kleingerechneten Bildern oder Grafiken (insgesamt bis $5 \mathrm{MB}$ ) an unsere Bildredaktion. Wir werden zeitnah eine Entscheidung treffen und mit euch in Kontakt treten. Über Zusendungen, Tipps und Kontakte freut sich die:

bildredaktion@soziologiemagazin.de

EINSENDESCHLUSS:

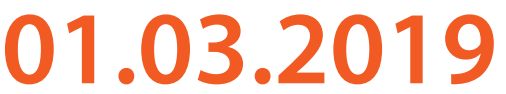




\section{Konsum und Verhalte
postmaterialistischen \\ Gesellschaften}

Liebe Leser_innen,

Wir, die Redaktion des Soziologiemagazins, konsumieren zahlreiche Dinge im Zuge jeder Heftentstehung. Sei es der fair gehandelte Kaffee, den wir während des Lektorats von Texten konsumieren, sei es unser Notebook, auf dem wir arbeiten und mit dem wir via Skype Redaktionssitzungen abhalten oder seien es Bücher und Artikel, um Inspirationen für neue Call4Papers zu erhalten. Es sind diese und viele weitere physische Güter, Textverarbeitungsprogramme und andere Technologien sowie vor allem viel Zeit, die wir für jedes Heft konsumieren.

Konsum begleitet uns überall hin und ohne es zu bemerken, konsumieren wir rund um die Uhr. Gleichzeitig errichten sich um uns immer weiter in rasanter Geschwindigkeit Systeme, die Konsum fördern, verbessern und optimieren. Dabei ist Konsum nicht nur ein Verhalten, Konsum kann gleichwohl als etwas bezeichnet werden, was wie eine Sprache funktioniert, die uns selbst formt und deformiert, lenkt, in unseren
Gedanken und Handlungen. Gleichwohl ist Konsum zunächst eine bloße Idee, welche in Subjekten entsteht und ihre symbolische Kraft in Gemeinschaften entfaltet. Um Konsum herum entstehen Praktiken des Konsums, aber auch antikonsumistische Praktiken. Dies wirft soziologisch die Frage auf, inwieweit wir uns dem Konsum entziehen, ihn gesamtgesellschaftlich durch unser Verhalten steuern sowie verändern können und wie wir überhaupt konsumieren möchten. Aus diesen Fragestellungen heraus entstand ein Heft zum Thema Konsum, mit ganz unterschiedlichen Schwerpunkten. So haben wir Beiträge gesammelt, die sich beispielsweise mit Nachhaltigkeit, mit Souveränität und mit Formen des Widerstands befassen.

Zum Einstieg führt Tamara Schwertel ein Expert_inneninterview mit dem Konsumsoziologen Kai-Uwe Hellmann. Im Gespräch werden aktuelle Forschungsagenden, aber auch Herausforderungen und zukünftige Forschungsperspektiven der deutschen Konsumsoziologie 
thematisiert. Hellmann diskutiert subjektzentrierte Fragen und Ansätze in der Konsumsoziologie und äußert sich zur Rolle des Subjektes.

Das graphische Essay Konsumierend schreiten wir voran? von Yen Sulmowsky leitet unser Schwerpunktthema ein. Yen setzt sich mit den Themen des nachhaltigen Konsums und der sozialökologischen Transformation aus einer praxissoziologischen Perspektive auseinander. Eine zentrale Überlegung ist, ob gesellschaftliche Transformation durch individuelle Konsumentscheidungen erreichbar ist. Dazu benötigt es nach Yen die Einbettung in soziale Praktiken, was er anhand des Beispiels des Wasserverbrauchs verdeutlicht. Enrico Hoerster zeigt in seinem Beitrag Führe mich sanft ans gleißend helle Licht die Konsequenzen des nudging für die Struktur des Konsument_innenverhaltens. Dabei zeigt er auf, wie Normen zur Privatheit durch nudging unterlaufen werden, mit dem Ziel, ein möglichst umfassendes Bild über die jeweiligen Nutzer_innen zu erfassen. Ob der sukzessive Verlust von Privatheit wünschenswert zugunsten eines optimierten Angebots ist, bleibt dem Urteil der Leser_innen überlassen.

Neben dem Verlust von Privatheit wird auch die Souveränität von Konsument_ innen von unseren Autor_innen infrage gestellt. In Eine „Opposition“ gegen „Autoritätssysteme" des Marktes? geht Soonim Shin zunächst davon aus, dass Konsument_ innen kaum Einfluss auf die Produktion aufgrund von fehlendem Wissen haben und so ihre Souveränität einbüßen. Die Autorin verdeutlicht jedoch anhand der amerikanischen Graswurzel-Initiative, wie kleine Produzent_innen Marktanteile erobern und so den Konsument_innen helfen, eine Angebotsvielfalt zu schaffen und ihre Interessen durchzusetzen.

In unserem Perspektiventeil werfen wir mit zwei Artikeln einen Blick über den konsumsoziologischen Tellerrand. Mara Mürlebach zeigt mit Ansätzen der politischen Geographie wie Konsument_innenverhalten durch die Platzierungen von Botschaften in Supermärkten beeinflusst werden können. In Shopdropping stellt sie ihre autoethnographische Studie vor und schildert eindrücklich ihre eigenen Erfahrungen. Shopdropping kann ihr zufolge als soziale Praktik des Widerstandes gegen den materialistischen Mainstream verstanden werden, bedenkliche Arbeitsund Produktionsverhältnisse aufzudecken. Diese Art von künstlerischem Aktivismus hat der Autorin zufolge das Potential der Sichtbarmachung von gesellschaftlichen Produktionsbedingungen und dem Enthüllen von Orten des Schweigens.

Beschäftigt man sich mit Konsum, kapitalistischen Strukturen und der Produktion von Dingen, sollte auch die marxistische Perspektive nicht fehlen. Im Beitrag Die gemeinschaftsbildende Funktion des Konsumierens bei Marx, Bourdieu und Laclau/ Mouffe zeichnet Valerie Scheibenpflug die 
Entwicklung marxistischer hin zu postmarxistischen Theorien des Zusammenspiels von Konsum und Gesellschaft nach. Dies geschieht anhand der Autor_innen Karl Marx, Pierre Bourdieu, Ernesto Laclau und Chantal Mouffe. Dabei bleiben für sie die zentralen Fragen, wie Gemeinschaft aussehen sollte, wie konsumiert und wie produziert werden sollte.

Veronika Riedl hat eine Literaturübersicht zu konsumsoziologischen Klassikern, Standardwerken und aktuellen Studienwerken zusammengetragen. Eine Auswahl interessanter anstehender Veranstaltungen und Tagungen hat Andreas Schulz recherchiert.

Parallel zu unserer neuen Ausgabe, die immer wieder die Forderung nach bewusstem Konsum und Souveränität aufwirft, haben wir uns Gedanken zu unserer redaktionellen Arbeit gemacht. Zum einen haben wir beschlossen, erstmals redaktionsexterne Nachwuchswissenschaftler_innen in unseren Reviewprozess mit einzubinden, um unsere Redaktionsentscheidungen durch neue Perspektiven $\mathrm{zu}$ erweitern. Zum anderen haben wir uns entschlossen, die redaktionelle Arbeit sichtbarer zu gestalten, um dadurch hervorzuheben, dass es sich beim Soziologiemagazin um ein kollaboratives Projekt zwischen den Nachwuchswissenschaftler innen und der Redaktion, die selbst Teil des wissenschaftlichen Nachwuchses ist, handelt. Jeder Beitrag, jeder Artikel, jede Rezension, jedes Interview wird von vielen
Personen im Zuge des Veröffentlichungsprozesses gelesen. Vom Zeitpunkt der Einreichung, über den Reviewprozess, zur Betreuung, bis hin zum Lektorat werden die Beiträge durchgesehen, kommentiert und es werden Anregungen gegeben, um den Artikel sowie das darin behandelte Thema sinnvoll zu erweitern. Wir möchten diese Veränderung vor allem zur Wertschätzung unserer redaktionellen Mitglieder nun erstmalig umsetzen und hoffen, so stärker den Prozess und die Arbeit, die hinter alledem steht, sichtbar zu machen und damit unseren Leser_innen weitere Einblicke in den Veröffentlichungsprozess zu geben.

Schließlich wünschen wir allen Leser innen viel Freude beim Lesen und Durchstöbern unserer 18. Ausgabe und bedanken uns herzlich für euer Interesse an unserem Magazin, für die fundierten, inspirierenden Diskussionen, die spannenden Einsendungen und für das Teilen unserer Beiträge sowie der Calls, die das Heft erst ermöglicht haben.

Stellvertretend für die Redaktion des Soziologiemagazins

Andreas Schulz und Tamara Schwertel

Das Editorial wurde von Tatiana Huppertz und Wibke Liebhart lektoriert. 
Editorial 1

Konsum und Verhalten in postmaterialistischen Gesellschaften | von Andreas Schulz und Tamara Schwertel

\section{Interview}

Mentaler Konsum 6

Ein Experteninterview mit Prof. Dr. Kai-Uwe Hellmann | geführt von Tamara Schwertel

\section{Schwerpunkt}

Konsumierend schreiten wir voran?.

Ein graphisches Essay zu Konsum und sozialökologischer Transformation | von Jedrzej (Yen) Sulmowski

Führe mich sanft ans gleißend helle Licht.

Konsequenzen des nudging für das Konsument_innenverhalten | von Enrico Hoerster

Eine Opposition gegen Autoritätssysteme des Marktes?

Wie kleine Produzent_innen Marktanteile erobern und so den Konsument_innen helfen | von Soonim Shin

\section{Perspektive}

Shopdropping 63

Materialities, Mobilities, Creative Interventions | von Mara Mürlebach

Die gemeinschaftsbildende Funktion des Konsumierens .77

bei Marx, Bourdieu und Laclau/Mouffe | von Valerie Scheibenpflug

\section{Aus der Redaktion}

Literaturübersicht zur Konsumsoziologie | von Veronika Riedl. 97

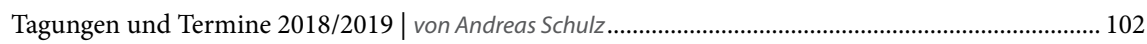

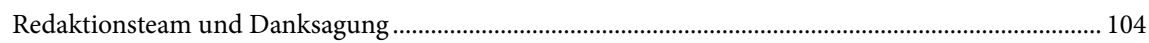

Impressum. 107

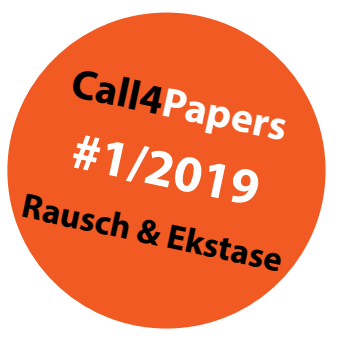

Wer würde nicht gerne in einem Magazin erscheinen?

Falls du gerne schreibst und Begeisterung für die Soziologie aufbringst, könnte in der nächsten Ausgabe dein Beitrag hier gelistet sein! 

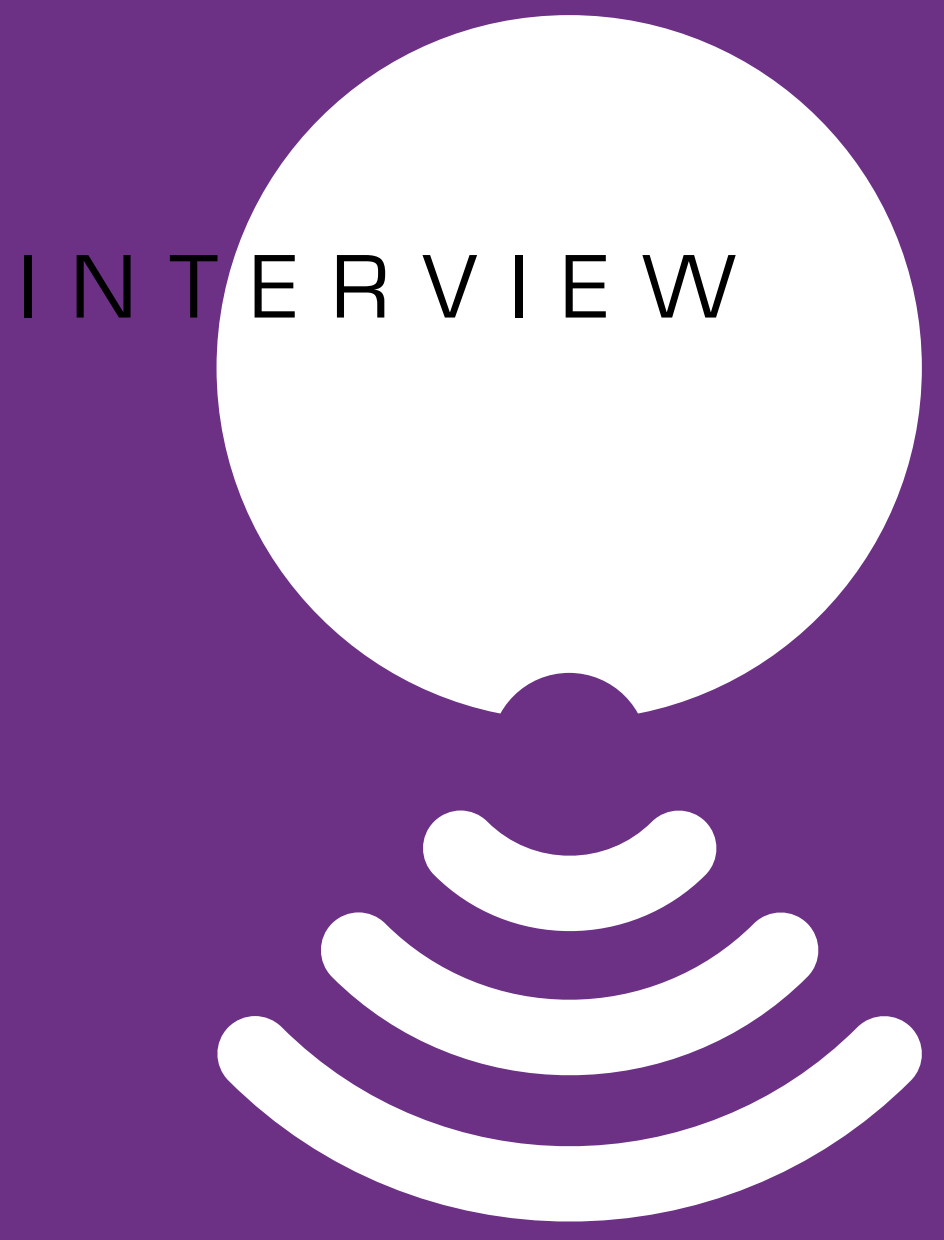


\section{Mentaler Konsum}

\section{Ein Experteninterview mit Kai-Uwe Hellmann}

von Tamara Schwertel

SozMag: Herr Hellmann, zum Einstieg möchten wir Sie fragen, was Sie heute schon konsumiert haben?

Hellmann: Um diese Frage angemessen beantworten zu können, ist vorab zu klären, was wir unter ,Konsumieren verstehen wollen. Setzt man allein schon beim Ressourcenverbrauch an, dann habe ich schon längst konsumiert, bevor ich morgens aufwache, weil mein Kühlschrank und mein Router im Dauerbetrieb laufen. Auch habe ich des Nachts mein Bett, meine Bettwäsche und meinen Schlafanzug konsumiert, ja selbst den Schutzraum ,Wohnung. Freilich ist dies ein anderer Konsum, als wenn ich mir in wachem Zustande meine übliche Kanne Kaffee morgens mache, mit der ich meine Tage möglichst immer beginne. Doch auch dies ist nur eine Variante von Konsum. Sprechen wir etwa von ,Erlebniskonsum, dann läuft simultan zu meiner Wahrnehmung der Welt ständig Konsum in mir selber ab, sowie ich wach werde, da ich auf bestimmte innere Erlebnisse aus bin, die ich durch die Nutzung bestimmter Sach- oder Dienstleistungen - aber auch nicht-kommerzieller Faktoren wie der Betrachtung der Morgenröte - in mir ermögliche und bewirke und die man als konsumierte Erlebnisse bezeichnen kann.

SozMag: Wie hat sich Konsum im globalen Norden entwickelt und wie unterscheidet er sich $z u$ anderen Regionen der Welt?

Hellmann: Wenn wir unter Konsum schlicht Lebenserhaltung begreifen, also die Befriedigung physiologischer Bedürfnisse im Sinne Abraham Maslows, dann dürfte es keine großen Unterschiede zwischen Nord oder Süd, Ost oder West geben. In dem Moment aber, wo wir diese Ebene des Überlebensnotwendigen verlassen, öffnet sich zunehmend eine Welt fast unbegrenzter Möglichkeiten. In der nördlichen Hemisphäre - aber ich bin mir gar nicht sicher, ob diese Fokussierung noch zweckmäßig ist, denken wir nur an Auckland, Buenos Aires, Kapstadt, Sydney - hat sich 
Der eigentliche ,Ort' des Konsums befindet sich in uns, alles andere sind äußerliche, wenngleich notwendige Rahmenbedingungen und Begleitumstände, wenn Konsum realiter geschieht: ein Vorgang, der für uns selber innerpsychisch aktiv Konsum bedeutet, wenn wirunsere Aufmerksamkeit genau darauf lenken.

\section{Ka i-Uwe Hellmann}

Kai-Uwe Hellmann, geboren 1962, studierte Philosophie und Politikwissenschaft. Er hat ein Diplom in Politologie 1989, wurde 1995 in Soziologie promoviert und habilitierte sich 2003 im gleichen Fach. Zurzeit ist er apl. Professor für Konsum- und Wirtschaftssoziologie an der TU Berlin. Sonstige Forschungsinteressen umfassen Gesellschaftstheorie, Militär- und Organisationssoziologie sowie Systemtheorie.

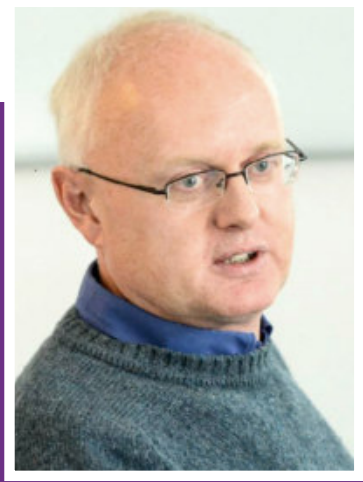
Ausgewählte Publikationen: Systemtheorie und neue soziale Bewegungen (1996), Soziologie der Marke (2003), Fetische des Konsums (2011), Die Gesellschaft des Konsums (2013).

ein Reichtum an Konsummöglichkeiten angesammelt, der in der Menschheitsgeschichte seines Gleichen sucht. Und was das spezifisch Nördliche daran ist? Ich vermute, es hat mit Geschichte, Klima, Kultur zu tun. Einen kleinsten gemeinsamen Nenner anzuführen fällt mir schwer. Massenproduktion und Massenkonsum haben sich zuallererst in Europa und Nordamerika verbreitet. Inzwischen aber, dies zeigt das Buch ,Herrschaft der Dinge' von Frank Trentmann (2018) recht gut, ist dieser anfänglich als singulär empfundene
Sonderstatus verloren gegangen. Heutzutage gibt es zwischen Weltmetropolen wie London und Tokyo oftmals größere Gemeinsamkeiten in Sachen Konsum, als wenn man Berlin mit der Uckermark vergleicht.

SozMag: In Ihrem Beitrag ,Das konsumistische Syndrom' (2008) beschreiben Sie Konsum als etwas, was auf das Subjekt einwirkt, es in seiner Lebensführung und in seinem Selbstwertgefühl beeinflusst. Können Sie für unsere Leser_innen, die Ihren 
Beitrag möglicherweise noch nicht kennen, kurz ausführen, was Sie damit konkret meinen? Wie verändert sich das Subjekt durch Konsum?

Hellmann: Die Formulierung, Das konsumistische Syndrom' stammt von Zygmunt Bauman aus dem Jahre 2004. Bauman hat damit eine Art Weltverhältnis bezeichnet, bei dem Konsum das Verhältnis zur Welt maßgebend prägt: Alles wird konsumiert, die Erwartungshaltung universalen Konsums gleicht Kleists , grüner Brille', mit der wir die Welt anschauen. Vorangegangen ist dem konsumistischen Syndrom nach Bauman übrigens das produktivistische Syndrom, bei dem die Welt - d. h. die Gesellschaft, die Beziehungen zu anderen Menschen und sich selbst - primär durch das Prinzip der Arbeit gedeutet wurde. Arbeit war im 19. Jahrhundert das zentrale Deutungsschema, um Ordnung in die Welt zu bringen. Im Laufe des 20. Jahrhunderts hat dieses Deutungsschema aber zusehends an Einfluss verloren, und was ihm nach Bauman gefolgt ist, war der Konsum. Hier stimmt Bauman übrigens mit Baudrillards ,Konsumgesellschaft' überein. Die Qualität der eigenen Lebensführung und das eigene Selbstwertgefühl werden demnach nicht mehr so sehr unter dem Gesichtspunkt der Arbeit betrachtet und beurteilt, sondern danach, was, wie, wo, mit wem und wozu wir konsumieren. Welche Veränderungen dabei die Menschen erfahren, die sich diesem Prinzip des Konsums weitgehend übereignen, kann nun ebenfalls mit Bauman beantwortet werden, wenn man etwa in sein Buch ,Leben als Konsum' (2017) hineinschaut. Dann steht für uns nicht mehr nur unsere Arbeitskraft im Mittelpunkt der Selbstbewertung, sondern inwieweit wir generell zur Ware für den Markt geworden sind. Oder um es mit Erich Fromm (2014) zu sagen: Wir unterwerfen unsere Lebensführung einer ,MarketingOrientierung;, bei der wir immer genau das tun, was uns in bestimmten Kreisen die meiste Anerkennung verschafft. Mit Georg Simmel (2014) könnte man auch sagen: Wir richten unser Leben immer nach der neuesten Mode aus.

SozMag: Was spielt beim Konsum und der Subjektkonstitution eine Rolle? Wer konsumiert wie und warum? Gibt es so etwas wie typische Konsumnarrative?

Hellmann: Was beim Konsum eine Rolle spielt? Dass er uns Genuss bereitet, so Hermann Heinrich Gossen, dass er uns bestenfalls glücklich macht, oder wenigstens doch satt und zufrieden. Und für die Subjektkonstitution ist Konsum so bedeutsam geworden, weil vor allen Dingen ihm, durch die Werbung massiv propagiert, die Potenz nachgesagt wird, für das eigene Selbstwertgefühl eine unübertreffbare Rolle zu spielen. Gemäß der Marketing-Orientierung rangiert Konsum ganz weit oben in der gesellschaftlichen Werteskala, sodass er nicht bloß 
für die unteren Bedürfnisschichten der Maslow'schen Pyramide Relevanz besitzt, sondern gerade an deren Spitze, wenn es um Selbstverwirklichung geht. Arbeit ist zwangsläufig zwar noch wichtig, irgendwo muß das Geld ja herkommen. Aber selbst für die Arbeit kann gesagt werden: Der Konsum der Anerkennung, die uns unsere Arbeit in den Augen anderer verschafft, wie auch der Konsum des Prestiges, das Aufstieg, Einkommen, Erfolg, Karriere, Macht vermitteln können, sollten, ist vielen enorm wichtig geworden. Insofern ist uns sogar Arbeit Mittel zum Zwecke des Konsums geworden. Und jenseits der Arbeitssphäre gibt es dann eine hyperkomplexe Mannigfaltigkeit an Aktivitäten, die wir in unserer Freizeit wahrnehmen und die wiederum (in der Regel) Konsum bedeuten. Typische Konsumnarrative sind dabei epochen- und generationenabhängig. Es gab sie zu allen Zeiten, sowie Konsum Autonomie zukam. Für viele von uns dürften das Glücksversprechen, das Konsum impliziert, aber auch Konsum als Kompensationsmedium, man denke hier an ,Trost der Dinge` von Daniel Miller (2014), oder die mit Konsum verbundenen Inklusionsaussichten fortlaufend Orientierung, ja Sinngebung vermitteln.

SozMag: In der Literatur wird davon ausgegangen, dass es bei Konsum nicht mehr primär um Konsumgüter, sondern vielmehr um dahinterliegende Werte, wie beispielsweise Nachhaltigkeit oder Gesundheit geht.
Wie strukturiert sich Konsum durch solche postmaterialistischen Narrative?

Hellmann: Sicher gibt es jede Menge Fachliteratur, in der behauptet wird, Werte wie Nachhaltigkeit oder Gesundheit besäßen einen besonderen Stellenwert für den Konsum. Das sei hier auch nicht bestritten. Aber es ist doch eher ein Minderheitenthema. Global betrachtet sind Grundversorgung, Lebensstandard, Teilhabe, Wohlstand eher vorherrschend. Wir hier haben inzwischen allerdings einen solchen Sättigungsgrad erreicht, dass plötzlich auch andere politische Themen wie Ausbeutung, Kinderarbeit, Umweltverschmutzung im Zusammenhang mit Konsum diskutiert werden. Und wenn von postmaterialistischen Narrativen die Rede ist: Für jene, die ein solches Prosperitätsniveau erreicht haben, dass ihnen solche Werte am wichtigsten werden, mutiert Konsum zur Bekenntnisfrage, zur Scheidung von Freund und Feind. Inwieweit die Protagonisten nachhaltigen Konsums tatsächlich nachhaltig konsumieren, steht dabei auf einem ganz anderen Blatt. Denn kaum etwas ist völlig unkontaminiert, nicht doch irgendwie nicht-nachhaltig, wenn es um Konsum geht: Die Komplexität der Interdependenzen jeder einzelnen Wertschöpfungskette ist erdrückend undurchdringlich. Die reine Lehre dürfte daher kaum zu einem reinen Konsum führen können. 
SozMag: In einem Ihrer Beiträge deuten Sie an, dass ein Bewusstsein über Werte wie Knappheit und Nachhaltigkeit nicht unbedingt praktische Konsequenzen hat. Warum folgen darauf so wenige Konsequenzen und weshalb übernimmt das Subjekt so wenig Selbstverantwortungen?

Hellmann: Warum nicht? Wegen dieser Komplexität. Es erfordert eine kaum erreichbare Kompetenz und sehr, sehr viel Zeit, um lückenlos zu überprüfen, wie man auf absolut richtige Art und Weise, tadellos soll. Wobei ja jede/r von uns ständig für alles Mögliche Verantwortung übernimmt. Inzwischen wächst sich dieses Verantwortungserfordernis von der Mikro- bis zur Makroebene aber so dermaßen aus, dass es in purer Überforderung und ganz schnell in Erschöpfung mündet. Der AttitudeBehavior-Gap ist ja ein altbekanntes Phänomen. Mit Simmel (1989) gesprochen, ist es die Tragödie der Kultur dieser Gesellschaft, dass das, was man individuell gerade noch so auf die Reihe bekommt, mit dem, was gesellschaftlich-global passiert und sich

ständig ändert, immer weniger Schritt halten kann. Diese Kluft wird rasend größer, wofür niemand die volle Verantwortung übernehmen kann, und angesichts dessen auch nur für einen kleineren Ausschnitt Verantwortung zu übernehmen, für sich, die eigene Familie, Freunden, Kollegen, Nachbarn, ist schon respektabel genug.

SozMag: Sie weisen neben rationalem und kompensatorischem Konsum auf eine weitere Kategorie in der Konsumforschung hin, nämlich die der Phantasien, Tagträume, innere Erwartungen und Wünsche. Was unterscheidet diese Art von Konsum von den anderen Arten und weshalb wird darauf so wenig in der Konsumforschung in Deutschland Rücksicht genommen?

Hellmann: Diese Lesart von Konsum, oben kurz schon als ,Erlebniskonsum 'tituliert, zeichnet sich dadurch aus, dass man die Wende zum Postmaterialismus konsequent zu Ende denkt und die Dimension des Mentalen für das Verständnis von Konsum zur Geltung bringt. Denn was und wie auch immer wir konsumieren, und sehr, sehr viel Zeit, um lückenlos zu überprüfen, wie man auf absolut richtige Art und Weise, tadellos und ideologisch einwandfrei konsumieren soll. 
ob Sach- oder Dienstleistungen, daheim oder im Urlaub, alleine oder mit anderen, achtsam oder verschwenderisch, so besteht doch der mitnichten kleinste gemeinsame Nenner darin, dass der Konsum letztlich in unseren Köpfen stattfindet, in uns, in unseren Vorstellungen, in der subjektiven Bewertung, inwieweit Soll und Ist, Wunsch und Wirklichkeit in Übereinstimmung sich befinden oder auch nicht. Konsum ist ja doch nur ein Wechselspiel von Erwartungen und Erfüllungen oder Enttäuschungen, so Tibor Scitovsky (1992) in ,The Joyless Economy؛. Wir sind es wohl gewöhnt, Konsum materialistisch zu denken. Aber eigentlich sind dies nur Mittel, deren Zweck sich allein in uns erfüllt, wenn wir der Auffassung sind: Das war jetzt ein schönes Konsumerlebnis, sei es ein Besuch in einem Kino oder Restaurant, sei es ein Abendessen mit Freunden oder ein Ausflug im Urlaub. Der eigentliche ,Ort' des Konsums befindet sich in uns, alles andere sind äußerliche, wenngleich notwendige Rahmenbedingungen und Begleitumstände dafür, wenn Konsum realiter geschieht: ein Vorgang, der für uns selbst innerpsychisch aktiv Konsum bedeutet, wenn wir unsere Aufmerksamkeit genau darauf lenken.

SozMag: Angesichts aktueller Entwicklungen hin zum Online-Marketing, zu individualisierten Produkten und hin $z u$ anwachsenden Auswahl- und Entscheidungsprozesses werden Individuen ganz neue Möglichkeiten zu konsumieren geboten. Was verändert sich durch Möglichkeiten des digitalisierten Einkaufs und inwieweit ist dabei möglicherweise von entfremdeter Konsumation zu sprechen?

Hellmann: Digitalisierung verstärkt das Moment der Virtualität, des Vorgestellten und Vorstellbaren. Digitaler Konsum spielt sich größtenteils im Mentalen ab. Es gibt zwar jede Menge technologischer Infrastruktur, Bildschirme, Tastaturen, Lautsprecher, die den sinnlichen Zugang, den Kontakt zur Welt ermöglichen. Aber der Reiz des Spielens oder Einkaufens im Netz hängt ja wesentlich nicht an diesen Technologien, sondern an den inneren Vorstellungswelten, die sich damit ausbilden. Ob dies übrigens mit Entfremdung zu tun hat, ist gewiss diskussionswürdig. Doch was besagt Entfremdung? Dass eine vollständige Identifikation mit dem und Aneignung dessen, was passiert und was mit uns währenddessen passiert, nicht mehr möglich ist. Dieser Umstand ist aber generell erfüllt. Insofern ist Entfremdung ein permanentes Begleitphänomen moderner Lebensführung, die Tragödie unserer Kultur. Digitaler Konsum könnte sogar die Illusion von verringerter Entfremdung erzeugen, weil die Gestaltungsmöglichkeiten in virtuellen Welten als viel größer wahrgenommen werden als in der realen Welt, die uns permanent Zwänge auferlegt. Gamification ist ein gutes Beispiel, oder auch der neue Spielberg-Film, Ready Player 
One' (2018) zeigt dies doch sehr schön: die Anmutung von Allmacht individueller Phantasien, die Kreation hochgradig idiosynkratischer Avatare, ein Austoben in einem Möglichkeitsraum, der unübertroffen vielfältig erscheint.

SozMag: Herr Hellmann können Sie uns zum Schluss sagen, vor welchen Aufgaben die Konsumsoziologie angesichts der hier skizzierten Entwicklungen in den nächsten Jahren stehen wird?

Hellmann: Das lässt sich kaum auf die Schnelle beantworten. Was die Konsumsoziologie in Deutschland angeht, so sind wir in den letzten Jahrzehnten arg ins Hintertreffen geraten und haben einen enormen Nachholbedarf, weil das Fach auch institutionell kaum noch Beachtung findet. Das erscheint mir als die dringendste Aufgabe: Anschluss an die internationale Forschung herstellen, wissen, was Sache ist. Auch ist unser Kenntnisstand um die Stellung des Konsums in der heutigen Gesellschaft noch ungenügend. Wenn Sie nach der Rolle des Konsums für die Subjektkonstitution fragen, dann rastet häufig sehr schnell eine vermeintliche Evidenz ein, so als ob wir längst durchschaut hätten, dass zwischen Konsum und Subjektkonstitution eine bedeutende Verbindung bestünde. Empirisch ist dies allerdings, wenigstens in Deutschland, nur sehr selten überprüft worden, also in vergleichender Hinsicht: Ist Konsum für die Subjektkonstitution
99

Ist Konsum für die Subjektkonstitution tatsächlich wirkungsmächtiger geworden als Arbeit, Familie, Politik, Recht, Religion, Sport?

tatsächlich wirkungsmächtiger geworden als Arbeit, Familie, Politik, Recht, Religion, Sport? Auch empfinde ich das aufgeheizte Diskussionsklima um ethischen oder nachhaltigen Konsum als problematisch, weil damit nicht nur Politik betrieben wird, sondern der je einzelnen Konsument derart viel Verantwortlichkeit zugeschrieben wird, oft ungeachtet ihres/seines sozialstrukturellen Standorts, dass entsprechende Bewertungen rasch moralisierend-polemisch-diffamierend wirken, anstatt echten Erkenntnisgewinn abzuwerfen. Dass mit unseren heutigen Konsumgewohnheiten, Stichwort,Wegwerfgesellschaft', zahlreiche ernstzunehmende Probleme verbunden sind, ist längst unbestreitbar geworden. Doch wie konsumsoziologisch darauf reagiert wird, erscheint mir oft suboptimal. Hier gibt es sicher noch Luft nach oben.

SozMag: Vielen Dank für das Gespräch! 
Das Interview wurde von Tamara Schwer-

tel vorbereitet und schriftlich von Herrn Hellmann im August 2018 ausgefüllt. Am Lektoratsprozess waren die Redaktionsmitglieder Andreas Schulz und Wibke Liebhart beteiligt.

\section{LITERATUR}

Baudrillard, Jean (2014): Die Konsumgesellschaft. Ihre Mythen, ihre Strukturen. Wiesbaden: Springer VS.

Bauman, Zygmunt/Vecchi, Benedetto (2006): Identity. Conversations with Benedetto Vecchi. Cambridge: Polity.

Fromm, Erich/Funk, Rainer (2018): Haben oder Sein. Die seelischen Grundlagen einer neuen Gesellschaft. München: dtv.

Fromm, Erich/Stapf, Paul/Funk, Rainer (2014): Den Menschen verstehen. Psychoanalyse und Ethik. München: dtv.

Hellmann, Kai-Uwe (2008): Das konsumistische Syndrom. In: Hellmann, Kai-Uwe/Zurstiege, Guido (Hrsg.): Räume des Konsums: Über den Funktionswandel von Räumlichkeit im Zeitalter des Konsumismus. Wiesbaden: VS Verlag für Sozialwissenschaften, S. 19-50.

Hellmann, Kai-Uwe (2018). Verbraucherleitbilder, Konsumerlebnisse und die mentale Dimension des modernen Konsums. Zum Menschenbild der akademischen Verbraucherforschung, in: Christian Bala (Hrsg.): Jenseits des Otto Normalverbrauchers. Verbraucherpolitik in Zeiten des ,unmanageable consumer“. Düsseldorf: Verbraucherzentrale NRW, S. 19-51.

Miller, Daniel/Jakubzik, Frank (2014): Der Trost der Dinge. Fünfzehn Porträts aus dem London von heute. Berlin: Suhrkamp.

Scitovsky, Tibor (1992): The joyless economy. The psychology of human satisfaction. New York: Oxford University Press.

Simmel, Georg (1989): Das Geld in der modernen Kultur. In: Simmel, Georg: Schriften zur Soziologie. Frankfurt am Main: Suhrkamp, S. 78-94.

Simmel, Georg/Holzinger, Michael (2014): Philosophie der Mode. Zur Psychologie der Mode. Zwei Essays. Berlin: Holzinger Verlag.

Trentmann, Frank (2018): Herrschaft der Dinge. Die Geschichte des Konsums vom 15. Jahrhundert bis heute. München: Pantheon. 
SCHWERPUNKT
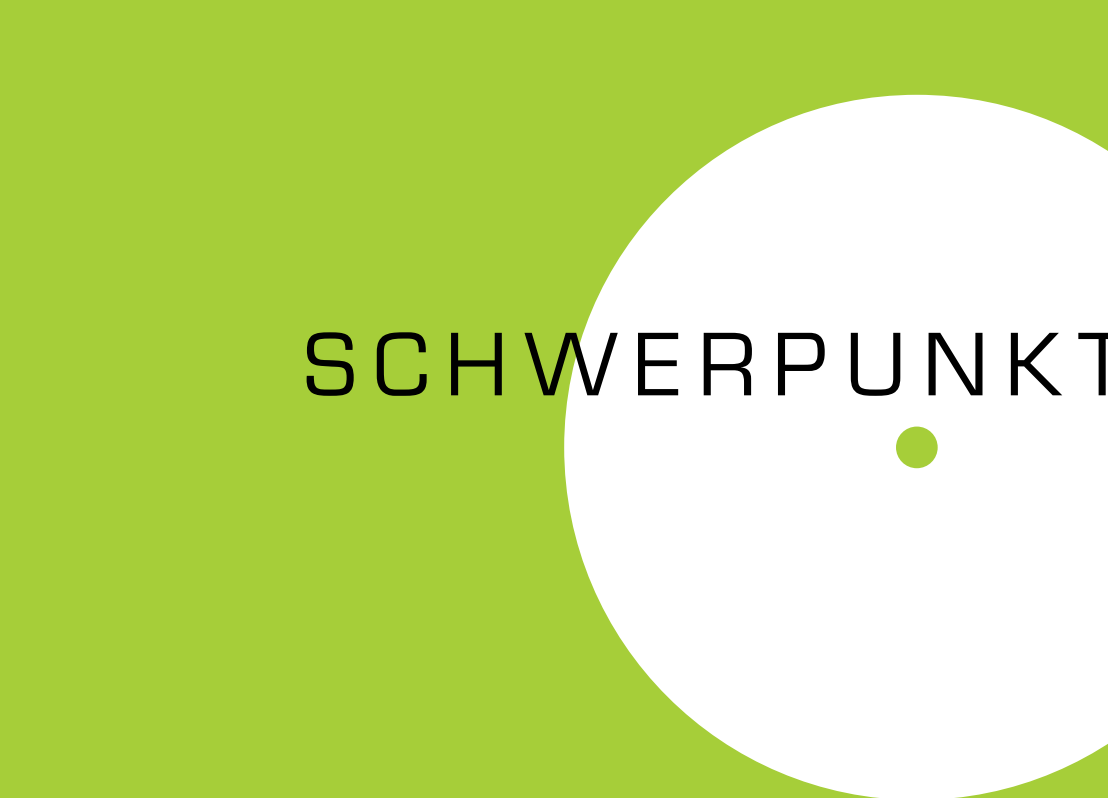


\section{Konsumierend schreiten wir voran?} Ein graphisches Essay zu Konsum und sozialökologischer Transformation.

Yen sulmowski

Die Erledigungen des Alltags

bewerkstellige ich zumeist

mit dem Fahrrad.

Für größere Besorgungen

nutze ich Car-sharing.

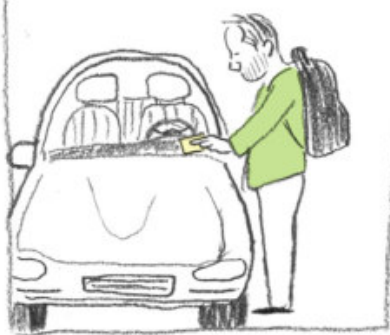

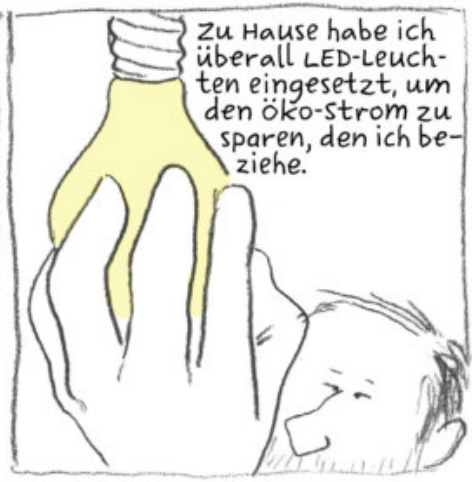

Ich weiß, wie es möglichst energiesparend zu heizen und zu luften gilt.

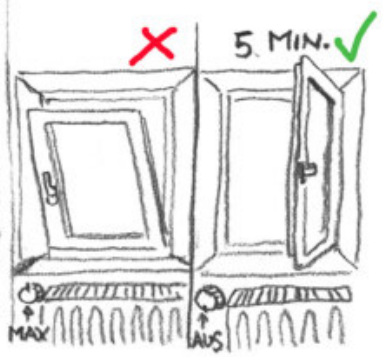

Zum Einkaufen packe ich (fast). Jedes Produkt steht für mich stellver-

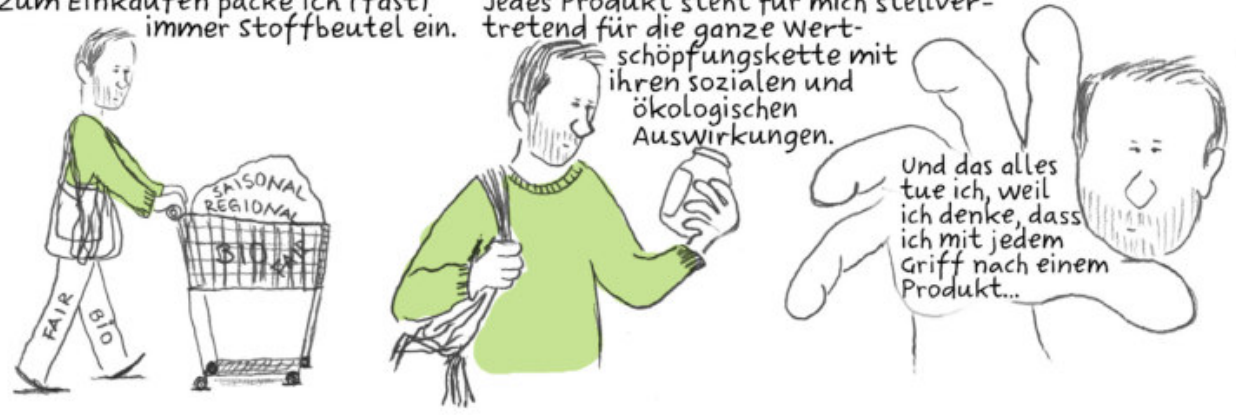



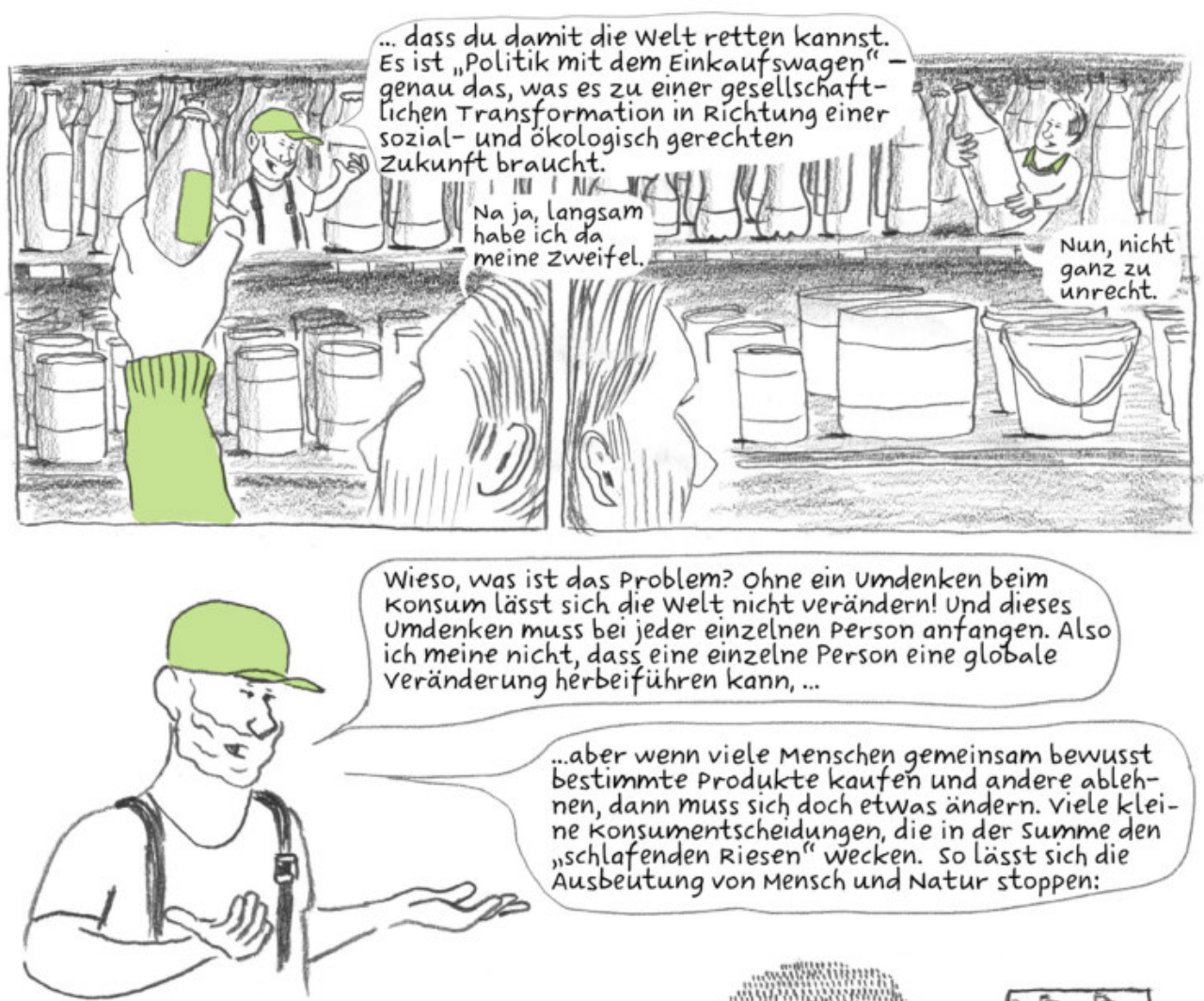

...aber wenn viele Menschen gemeinsam bewusst bestimmte produkte kaufen und andere ablehnen, dann muss sich doch etwas ändern. viele kleine konsumentscheidungen, die in der summe den "schlafenden Riesen" wecken. So lässt sich die Ausbeutung von Mensch und Natur stoppen:

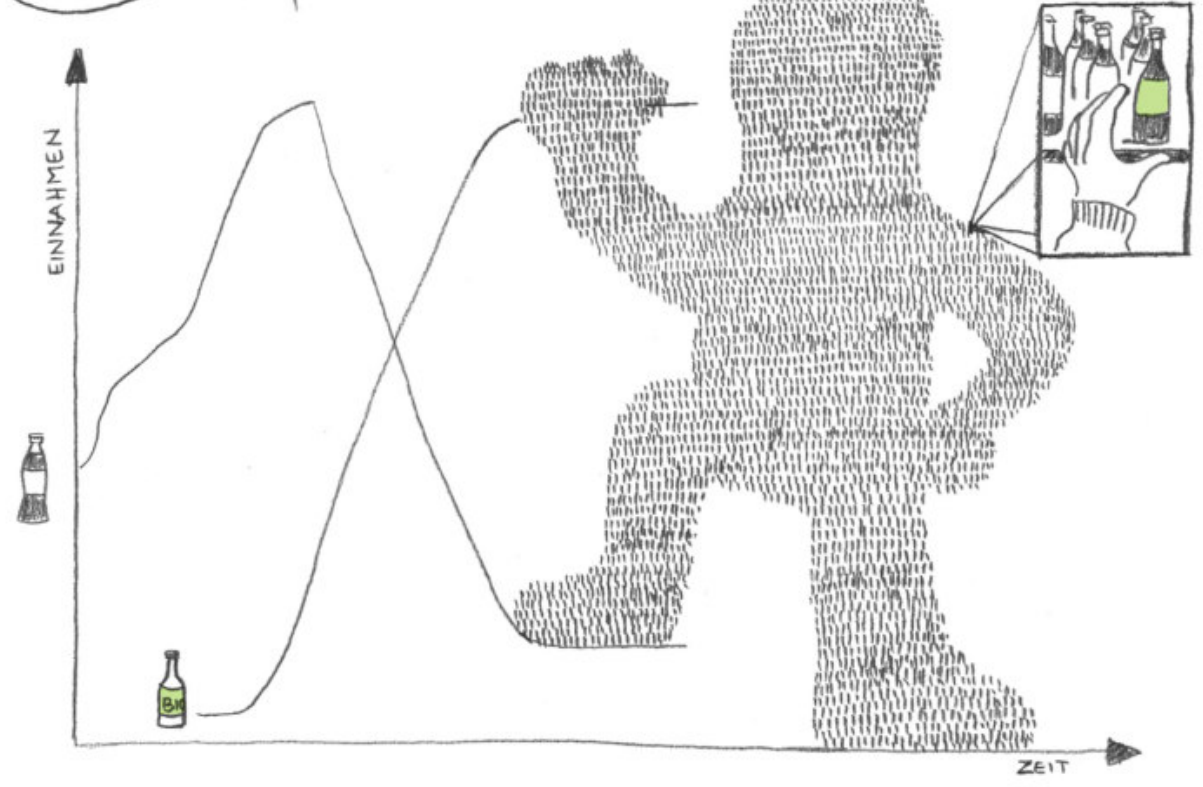


Ich sage nicht, dass sich die welt so nicht verändern lässt, aber ich möchte zu bedenken geben, dass sie sich womöglich in eine andere Richtung verändern wird, als du es dir wünschst. Und vor allem: was sich durch den individuellen konsum wahrscheinlich am wenigsten verändern lässt, ist das Ausmaß der Beanspruchung natürlicher Ressourcen oder der sozialen Ungleichheit. Deiner Vorstellung der Transformation liegt ein Modell eines generischen, also in zeit und Raum unveranderbaren Konsummenschen zugrunde, der rational handel $t, . .$.

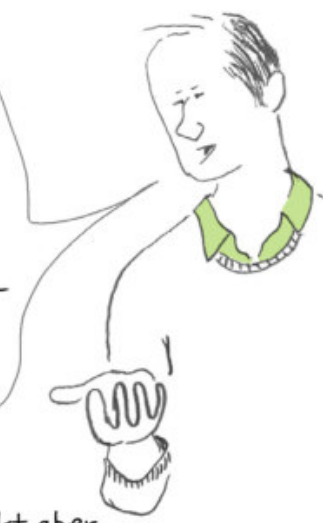

Dieses cehirn steckt aber

...d.h. Einsichten und informationen bewusst und direkt in sein Handeln. übersetzen kann - wie ein gesellschaftsloses cehirn.

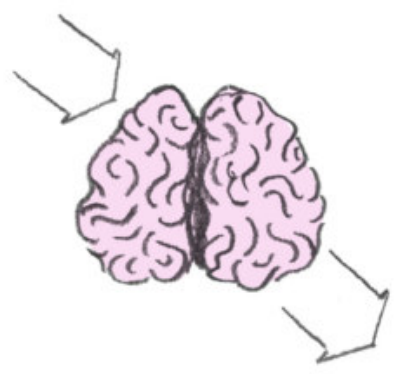

in einem körper, der als irgendein cieschlecht, mit irgendeinem Alter und mit irgendeiner Hautfarbe gesellschaftlich kodiert wird...

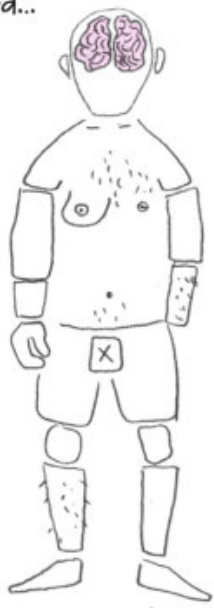

...und in bestimmten (machtförmigen) verhältnissen zu anderen menschen und Institutionen steht.

Die einzelnen Konsument *innen haben also aufgrund ihrer sozialen Positionen unterschiedliche voraussetzungen, ihr Handeln zu verändern. Dein Konsumriese ist also vermutlich ein Riese unter vielen.
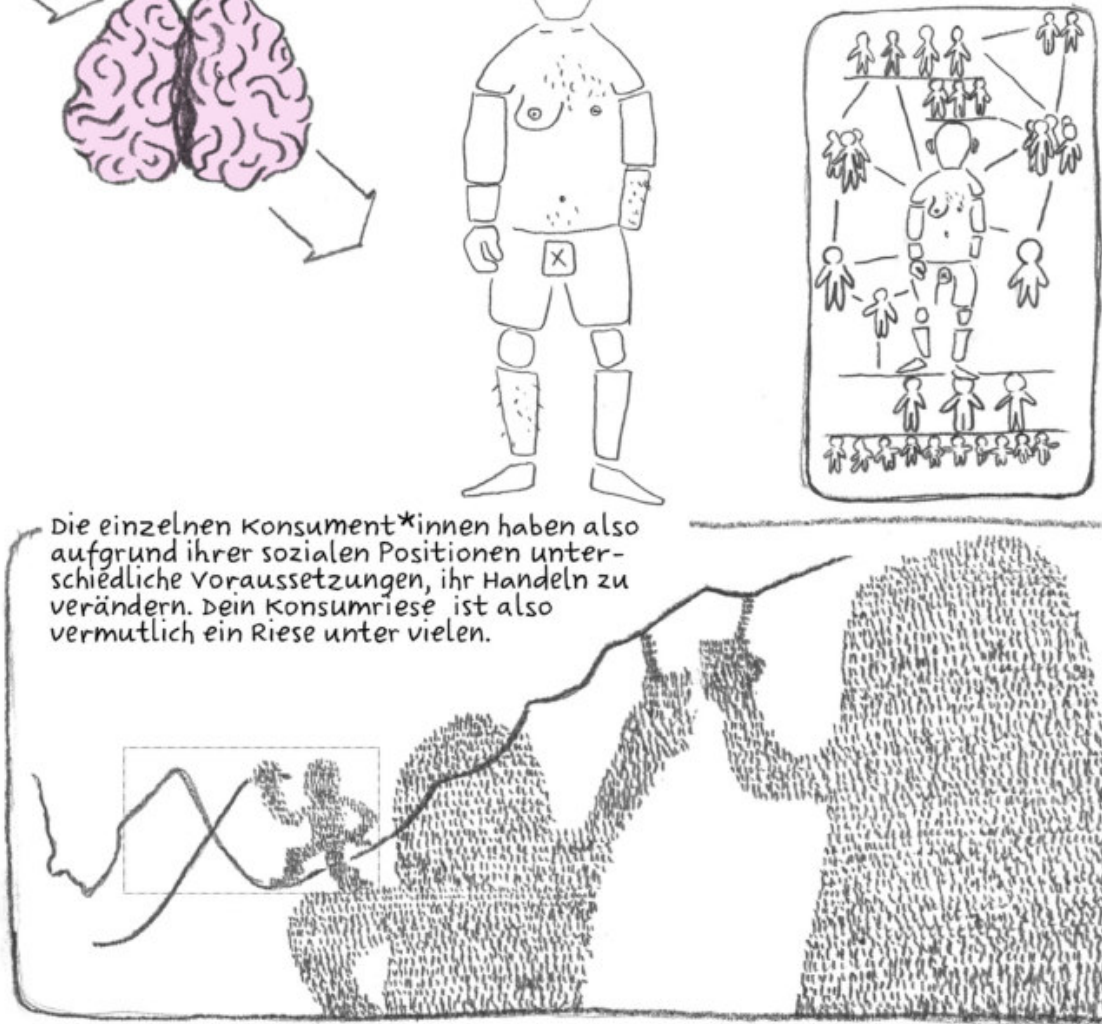


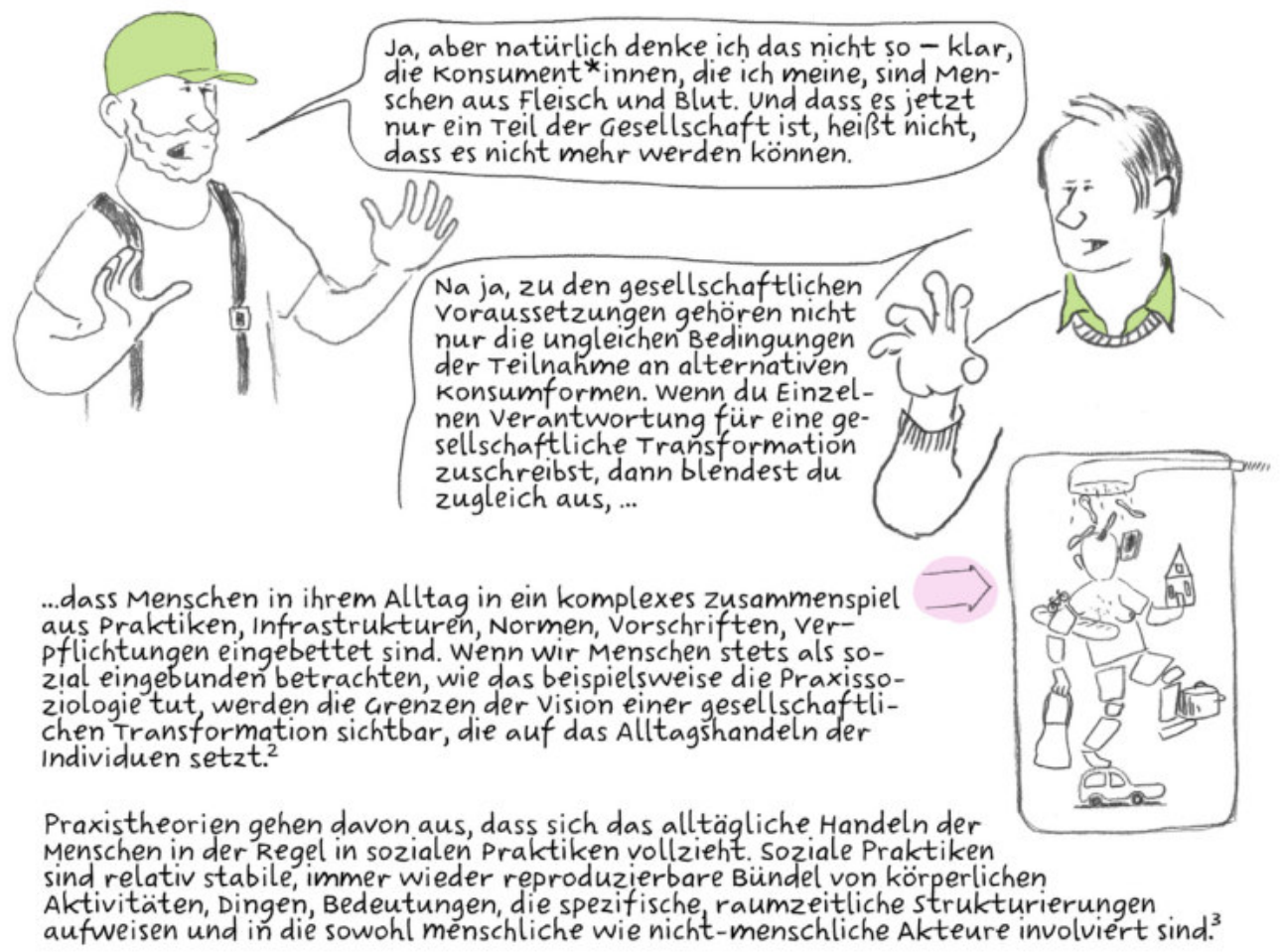

Aufgabe 1: Bilde aus den abgebildeten Elementen kohärente Sets, die sich als sozia-

- le Praktiken identifizieren lassen. Verbinde dazu die Elemente passend miteinander

mit Hilfe von Linien (Mehrfachverbindungen sind möglich).
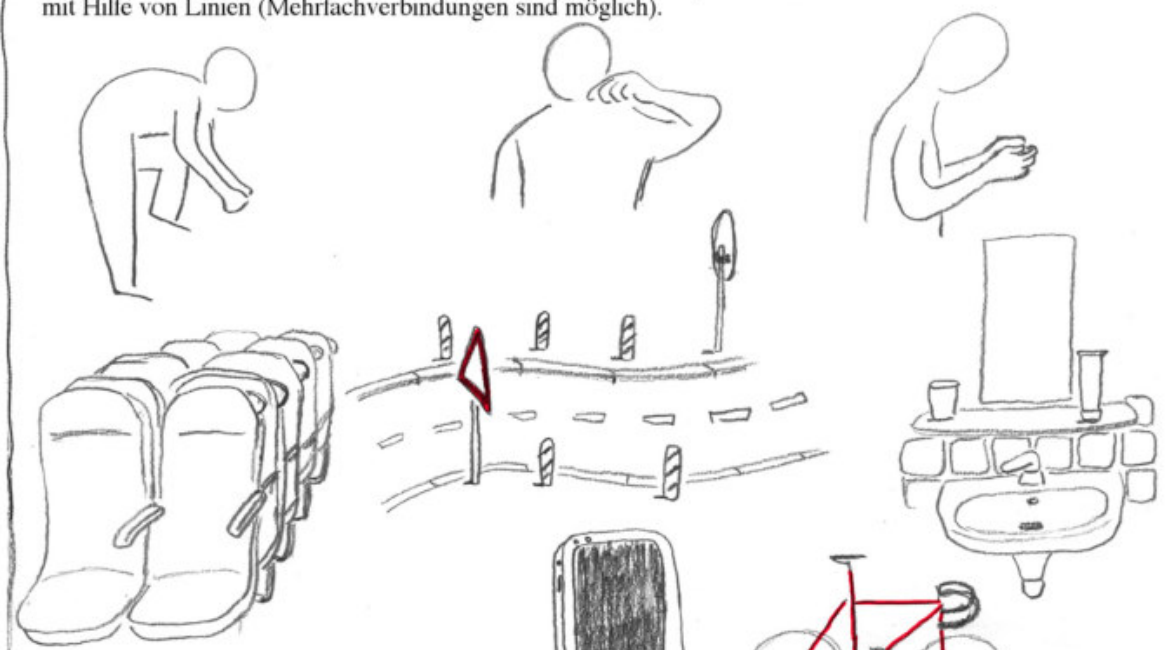

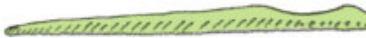

(1010)
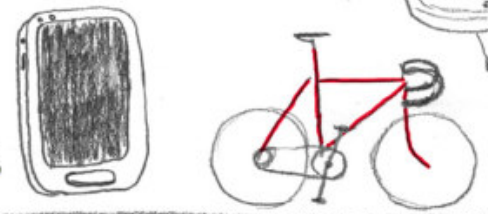
Die einzelnen verrichtungen spielen sich auf ihre je spezifische Art und weise nicht nur deswegen $a b$, weil wir daran denken, dieses oder jenes zu tun. Diese verrichtungen sind Teil eines zusammenhängenden situativen und zugleich historisch gewordenen soziomateriellen Arrangements. Der Alltag entspricht einem kontinuierlichen übergang von einer Praxis in die andere:
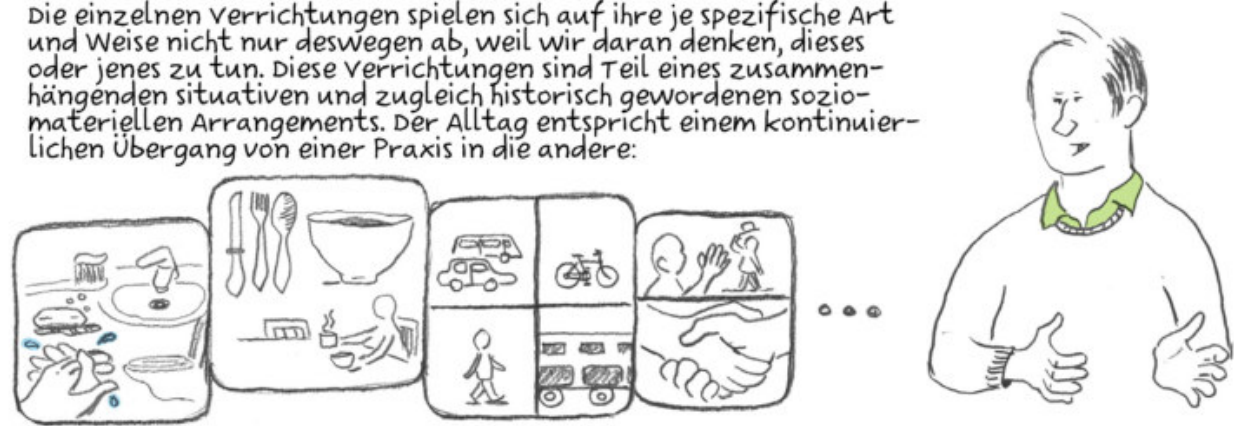

Was ich sagen will, ist, dass wenn wir individuelle verhaltensänderungen oder individuelle konsumentscheidungen in den Dienst einer gesellschaftlichen Transformation stellen, Wäre es sinnvoll, die Einbettung in soziale Praktiken mitzudenken. Hierzu ein Beispiel, und zwar eines, das nicht so manifest und symbolträchtig ist wie der Einkauf von BioLebensmitteln, sondern sich vielmehr im verborgenen des privaten Rückzugsraums abspielt: der wasserverbrauch. Die wassernutzung im Alltag ist vor allem ein Teil der Praxis der Nahrungszubereitung, des Wäschewaschens, des Putzens, des Toilettengangs sowie der Praktiken der körperpflege wie Händewaschen, Ganzkörperwäsche (Duschen, Baden), zähneputzen. Nehmen wir die Praxis der Körperwäsche und insbesondere die Praxis des Duschens. Diese Praxis lässt sich beschreiben, indem ihre menschlichen wie nichtmenschlichen Teilnehmer*innen, ihre sprachlichen wie nicht-sprachlichen Aktivitäten sowie ihre je eigenen zeitlichen und räumlichen strukturen in den Blick genommen werden. Zu der Praxis des Körperwaschens, wie sie aktuell (vornehmlich im globalen Norden) ausgestaltet ist, gehoren:

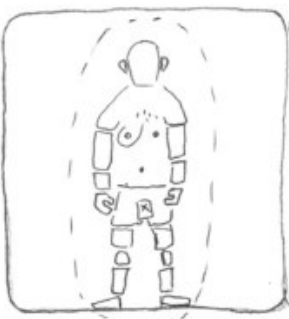

der menschliche körper, der zumeist im Ganzen...

$$
\begin{aligned}
& \text { Mo Dis Mi Do Fr Sa So } \\
& M_{0}^{\Delta} D_{\delta}^{\Delta} M_{i}^{\Delta} D_{0} F_{\gamma}^{\Delta} S_{a}^{\Delta} S_{j}^{\Delta} \\
& M_{0}^{\Delta} \text { Di Mi Do Fr } S_{s}^{\Delta} S_{0}
\end{aligned}
$$

...regelmäßig (je nach Jahreszeit, Beruf, Alter, Freizeitaktivitäten, etc.)...

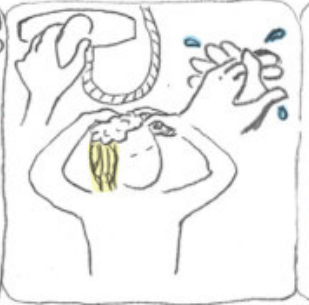

...mit Hilfe spezifischer eingeuibter Bewegungen...

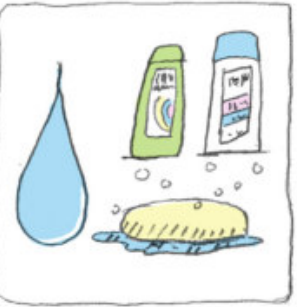

...mit wasser und mit Waschmitteln behandelt wird.

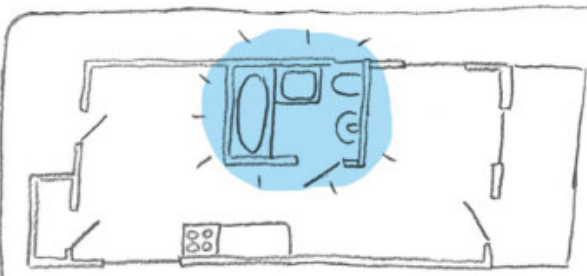

In der Regel findet diese Tätigkeit in eigener wohneinheit, in einem separaten

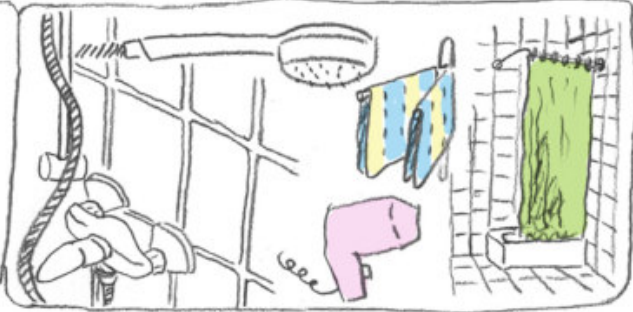
Raum statt, ... 
Es dürfen bei der Beschreibung der Praxis nicht diejenigen Elemente vergessen werden, die diese Praxis ermöglichen, ohne unmittelbar wahrgenommen werden zu können: ${ }^{\text {" }}$

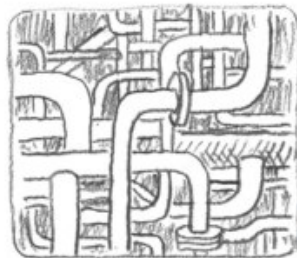

die Wasser- und AbWasserleitungen, ...

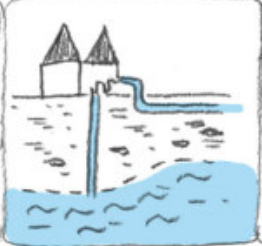

... die Wasseraufbereitungs- und Pumpanlagen...

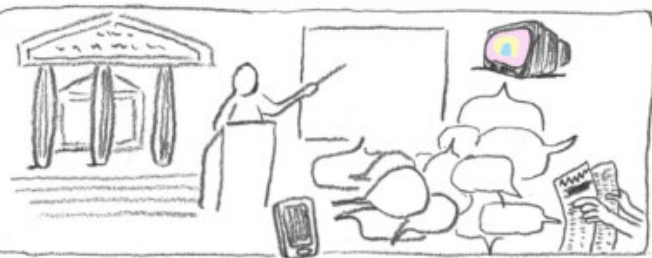

...sowie die Institutionen, die durch ihr entsprechendes know-how diese infrastruktur am Laufen halten und für die Reproduktion und Weiterführung der Wissensdiskurse sorgen.
Diese Praxis präfiguriert das verhalten der Menschen im Hinblick auf den Umgang mit ihren Körpern und geht mit dem heutigen historisch hohen Wasserverbrauch einher.
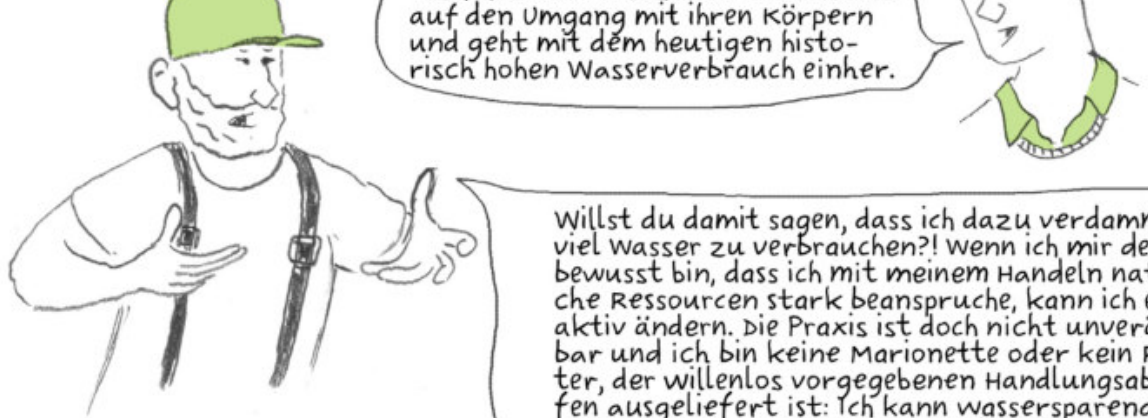

Willst du damit sagen, dass ich dazu verdammt bin, viel wasser zu verbrauchen?! Wenn ich mir dessen bewusst bin, dass ich mit meinem Handeln natürliche Ressourcen stark beanspruche, kann ich es doch aktiv ändern. Die Praxis ist doch nicht unveränderbar und ich bin keine Marionette oder kein Roboter, der willenlos vorgegebenen Handlungsabläufen ausgeliefert ist: lch kann wassersparende Duschkopfe einbauen, kurz und kalt duschen oder seltener duschen!

\section{Aus praxistheoretischer sicht sind menschliche Teilneh-} mer *innen von Praktiken nicht vollkommen determiniert. Die Praxis präfiguriert ihre Handlungen jedoch, sie lässt sie in bestimmten Bahnen ablaufen:
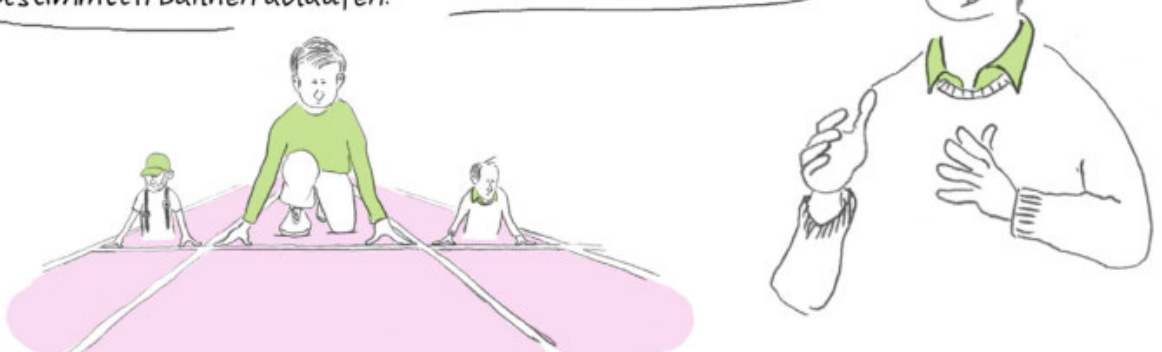

Das heißt, wir sind nicht gefangen in Praktiken, können diese aber a uch nicht beliebig verändern. Diese Beharrlichkeit ergibt sich daraus, dass soziale Praktiken eine Geschichte haben, die individuelle Handlung übersteigen. Der heutige individuelle Akt des Duschens spielt sich in einer bestimmten historischen konstellation ab, die das vorläufige Ergebnis gesellischaftlicher Entwicklungen ist. Im 19. und am Anfang des 20. Jahrhunderts war die praxis des körperwaschens noch anders ausgestaltet. zu ihr gehörten in der Regel: 


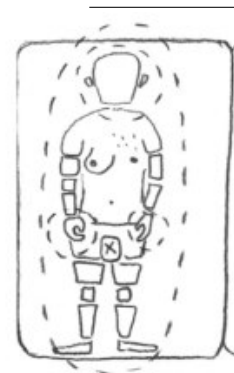

der menschliche körper, der zumeist stellenweise (vor allem an Händen und am Gesicht)...

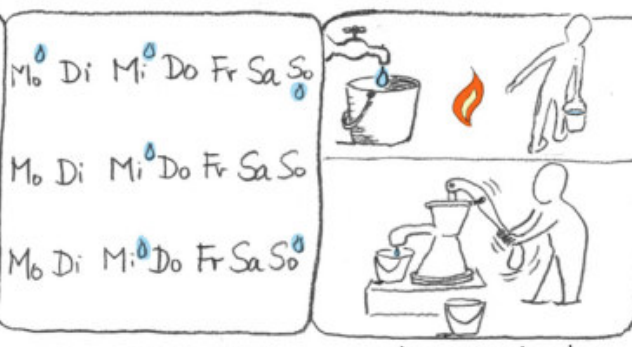

... je nach zugehörigkeit zu gesellschaft licher schicht regelmäßsig oder sporadisch...
... unter Einsatz der Arbeit des Wasserholens und -erhitzens (deren Art und Ausmaßje nach sozialer schicht variierte)...

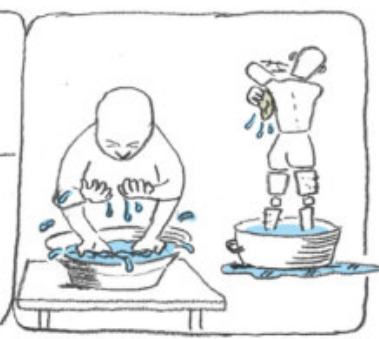

...mit Hilfe spezifischer Handbewegungen, mit Wasser einem waschlappen und oftmals mit seife behandelt wurde.

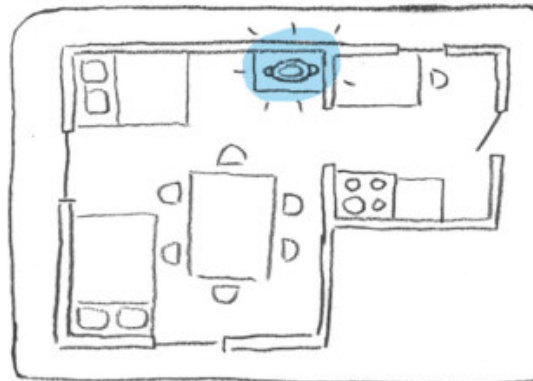

Wenn es nicht eine cianzkörperwäsche in einer öffentlichen Badeanstalt war, fand diese Tätigkeit in der Regel in der eigenen Wohneinheit statt,...

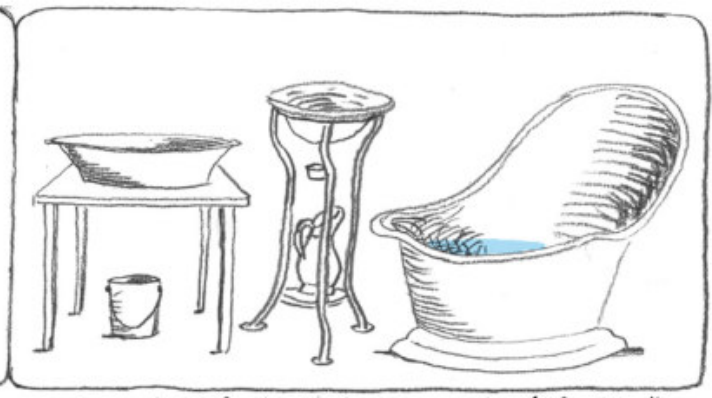

...Wo je nach Größe der Wohnung waschgef äße für die zeit der Wäsche bereitgestellt und hinterher beispielsweise unter dem Bett verstaut wurden oder wo eine Ecke für das Waschen permanent vorgesehen War?
Indem die Entwicklung nachgezeichnet werden kann, die von...

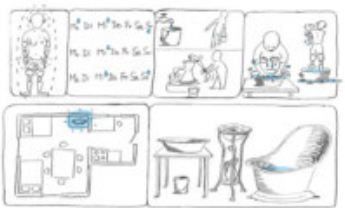

2u

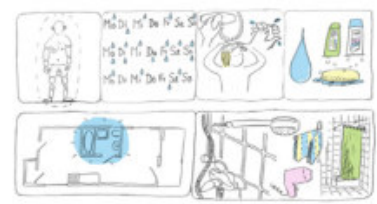

... führt, lässt sich verstehen, inwiefern die praxis des Duschens der individuellen verhaltensänderung im Hinblick auf den Wasser- und Energieverbrauch Grenzen setzt.

Eine der zentralen Entwicklungen hierbei waren Diskurse, die seit etwa dem Ende des 18. Jahrhunderts um den menschlichen körper kreisten: Der medizinische und volksgesundheits"-bezogene Diskurs, der den körper als Quelle von Krankheiten betrachtet...

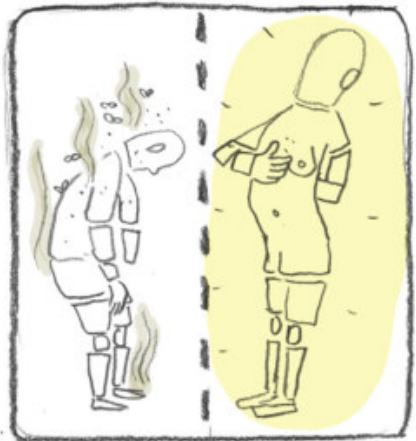

...und ihn vor diesem Hintergrund als ciegenstand sanitärer intervention darstell $t$, die u.a. auf die Erhöhung körperlicher vitalität abziel $t^{8}$ so entstehen Ratgeber, Maßsnahmen und Empfehlungen, wie und wie oft der körperzu reinigen ist?

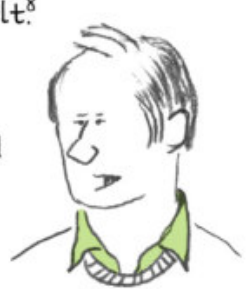




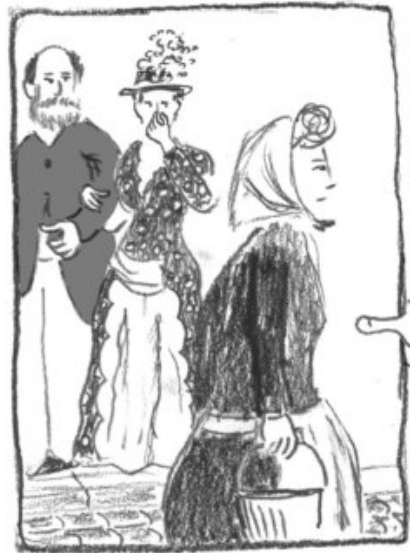

Mit diesen Entwicklungen geht eine veränderte wahrnehmung von körper und Reinlichkeit einher. Bestimmte äußere Hautbeschaffenheiten oder körpergerüche, die vormals nicht oder als wenig störend nen und Riuckstïndigen. nen und Rückstandigen. Schließlich avanciert eine gewissenhafte Reinhaltung des gesamten körpers zu einer bürgerlichen Tugend und wird zu einer voraussetzung der Teilnahme am gesellschaftlichen Leben überhaupt: Die regelmäßige cianzkörperwäsche wird zur Normalität.10
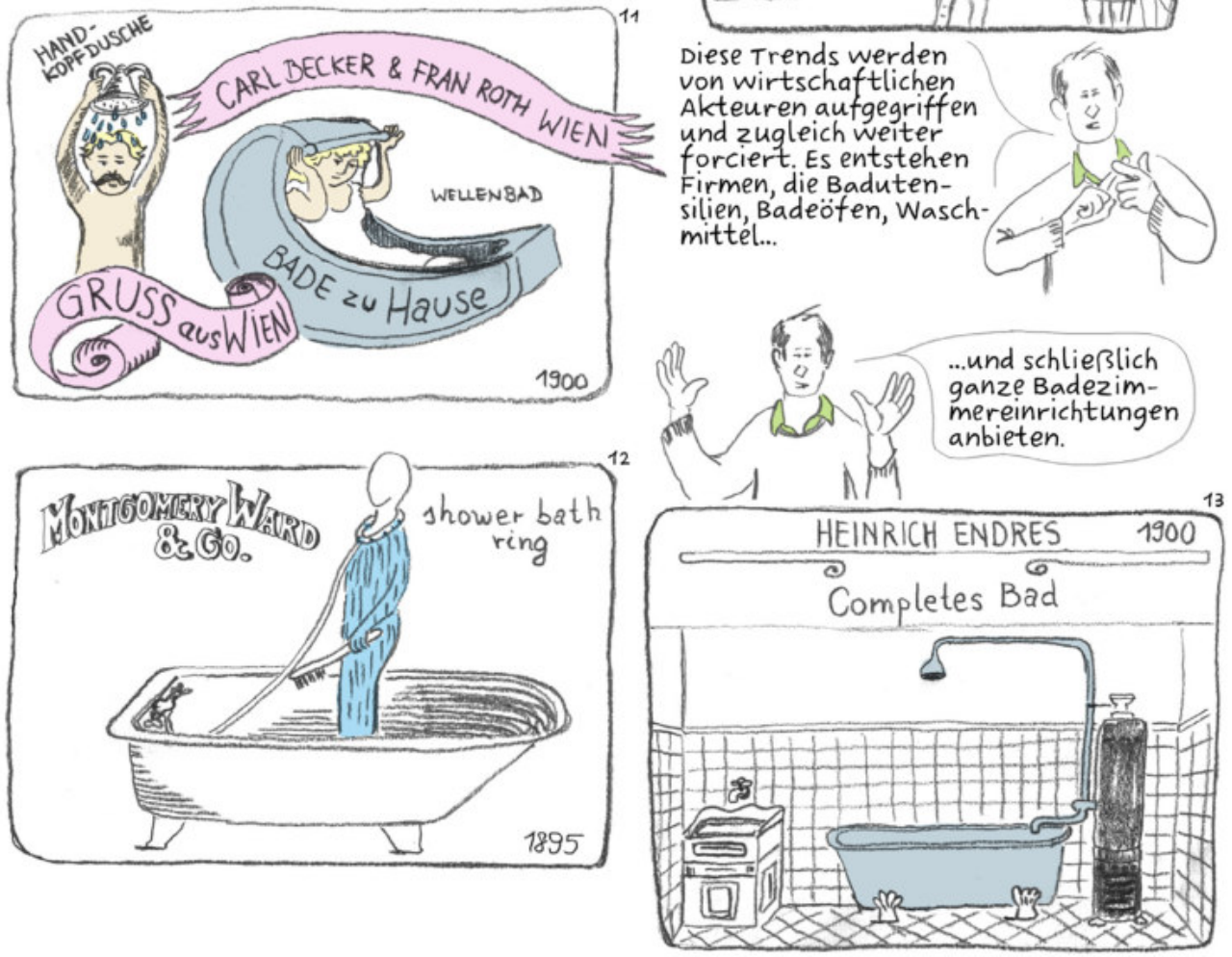


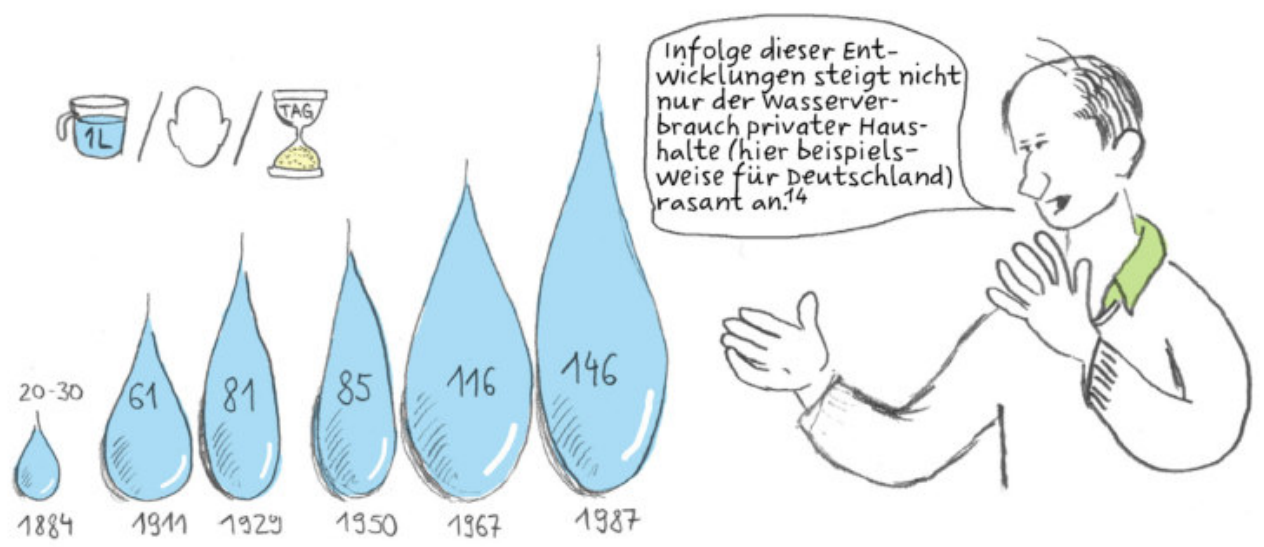

Der Ausbau der Kanalisation, die Ausstattung jeder einzelnen Wohneinheit mit infrastruktur, die Nutzung von fließendem wasser zu jeder zeit ermöglicht, steigert die Beanspruchung metallischer und mineralischer Ressourcen. Die Einführung des Badezimmers in architektonische standards erhöht die Inanspruchnahme von fläche. Die Wasserversorgung und - entsorgung erfordert höhere Pumpleistungen, wodurch der Energieverbrauch zunimmt.

Wenn ich dusche dann ist es zWar eine individuelle Handlung, in die aber bestimmte gesellschaftliche verhältnisse und Entwicklungen - wie ich sie weiter oben darstellte eingeschrieben sind. Das heißt, dass der Akt des Duschens nicht nur so ist, wie er ist, weil ich mich dazu entscheide, sondern weil er ein Teil einer sozialen Praktik ist, deren ceschichte viel früher anfängt als der Akt selbst.

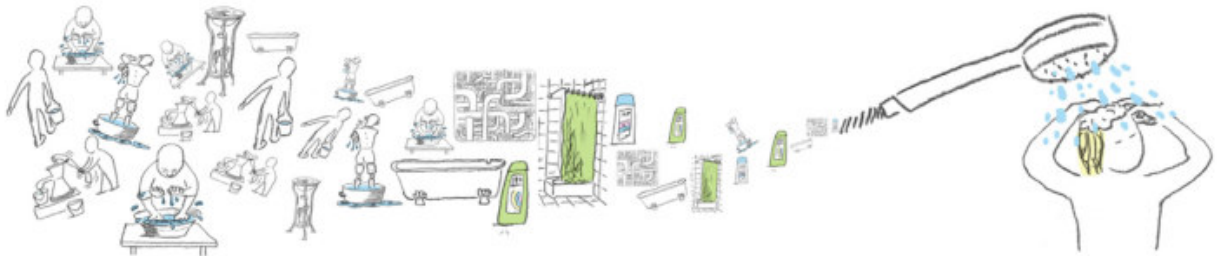

Aber es muss doch nicht die ganze praxis umgekrempelt werden, um umwelt- oder sozialrelevante veränderungen herbeizuführen. So ist beispielsweise der Wasserverbrauch in den letzten 30 Jahren deutlich gesunken und das wäre ohne die individuellen Bemühungen, Wasser zu sparen, doch nicht moglich gewesen. Wenn ich deine statistik ergänzen darf: 15

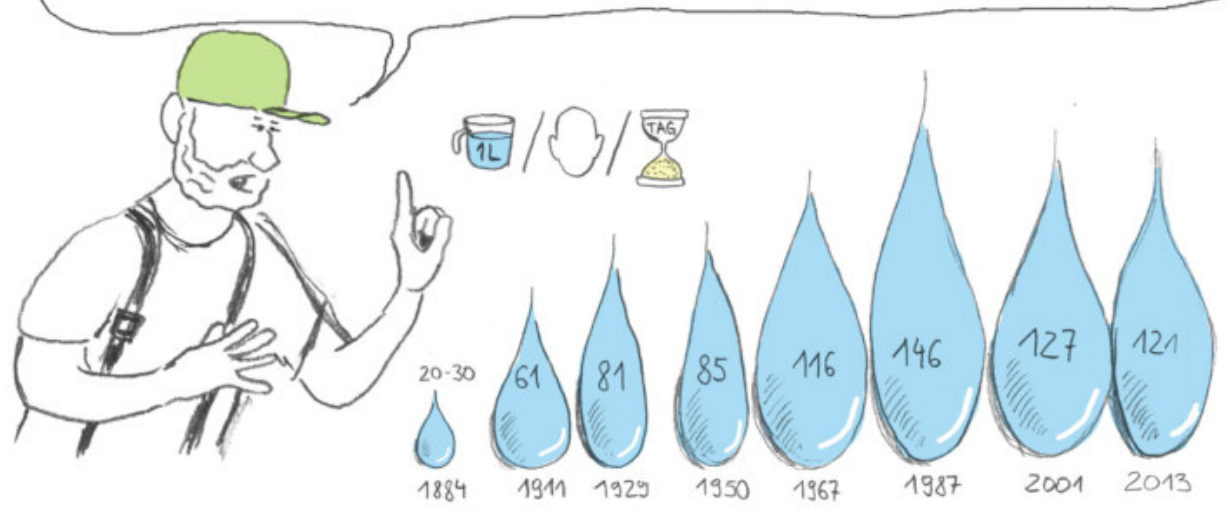


Ja, aber vermutlich passiert das im Rahmen der geltenden standards, d.h. die Praxis setzt der Reduktion eine Grenze. Ich schätze, dass der Wassserverbrauch nicht viel mehr abnehmen wird. Und im übrigen ist es fraglich, ob wassersparendes verhalten einen umweltrelevanten Effekt hat. Denn die Praktik des Duschens ist auf eine bestimmte infrastruktur angewiesen, die ihre eigenen materiellen, technischen oder. ökonomischen Pfadabhangigkeiten hat. So sind Abwasserleitungen auf eine bestimmte Durchflussmenge ausgelegt.
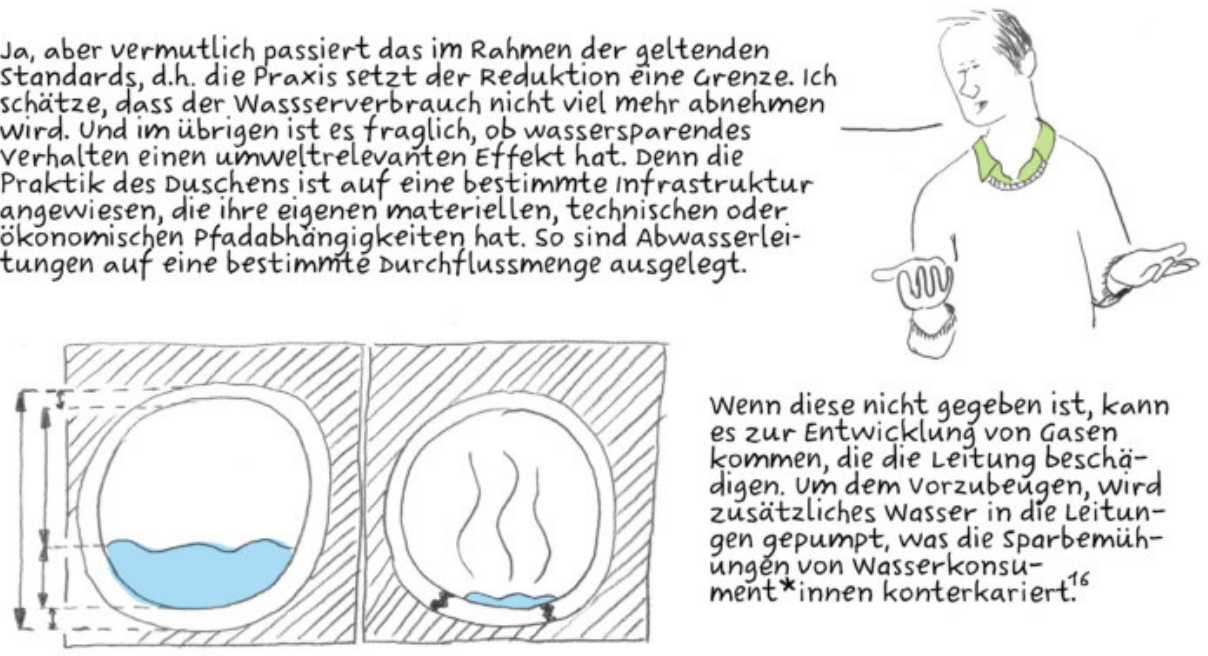

Wenn diese nicht gegeben ist, kann es zur Entwicklung von Gasen kommen, die die Leitung beschädigen. um dem vorzubeugen, wird zusätzliches Wasser in die Leitungen gepumpt, was die Sparbemühungenvon Wasserkonsument*innen konterkariert.
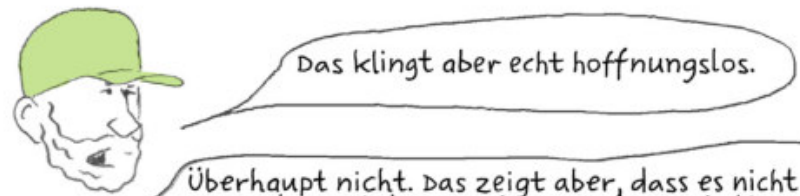

ist, ein Element der Praxis - z.B. das verhalten der menschlichen Teilnehmer *innen - zu verändern. Zugleich muss eine veränderung der infrastrukturen ${ }^{17}$ bzw. versorgungsregime ${ }^{18}$ stattfinden. Und damit meine ich nicht nur die technischen Aspékte der organisation von Produktion und Konsum (wie die Parameter der Wasserleitung), sondern auch die ökonomischen Aspekte. Und dieser wandel ist wiederum ohne die veränderung gesellschaftlicher Normalitätsvorstellungen kaum möglich. Dieșe standards und infrastrukturen sind nicht irgendwelche, sohdern historisch spezifische, d.h.

standards und infrastrukturen von kapitalistischen Gesellschaften. Die kapitalistische Wirtschaftsweise macht eine "imperiale Lebensweise" 19 die mit einem hohen Ressourcenverbrauch einhergeht, überhaupt erst möglich.

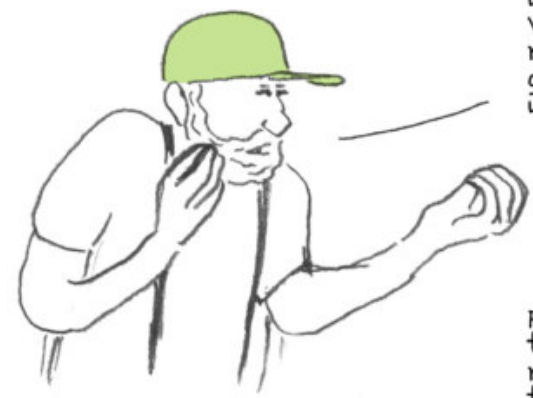

Ach, ist das etwa die alte Leier, dass der Kapitalismus überwunden werden muss, dass es kein richtiges verhalten im falschen System gibt? Ist so ein Denken nicht "kapitalozentrisch"!? ${ }^{20}$ Ist Kapitalismus nicht die große Erzählung, die die vielfalt von Produktionsund Konsumweisen nicht beschreiben kann, weil sie jegliches wirtschaftliches Handeln immer auf das verhälthis zwischen Eigentum an Produktionsmitteln, der Arbeitskraft und kapitalakkumulation reduziert? Wäre es nicht lohnend eher von einer Pluralität von Wirtschaftsweisen auszugehen? Dann ließe es sich denken, dass ne-

ben den verschiedenen kapitalismen auch andere Formen der (Re-)Produktion und des Gebrauchs existieren, wie die solidarische, commonale, die Care-ökonomie.21 Wirtschaftliches Handeln ist nicht nur kapitalistisch. So besteht mindestens die Hälfte der insgesamt geleisteten Arbeit in sorgenden Tätigkeiten,... 


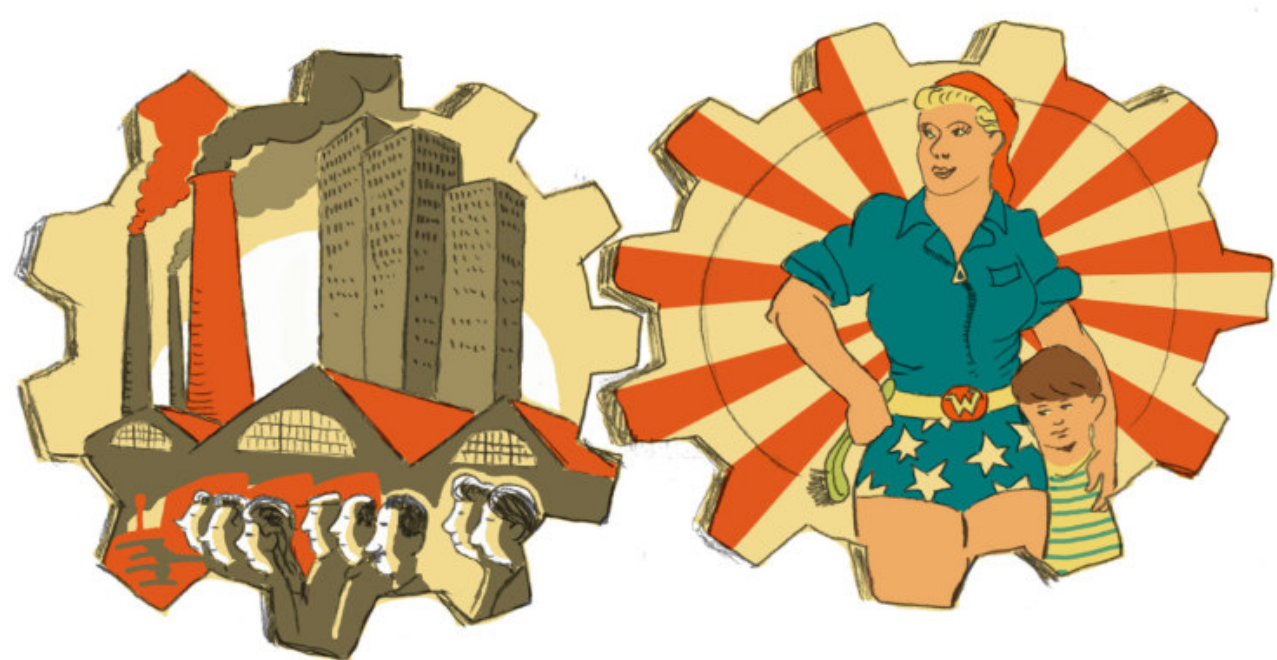

die eine arundlage jeglicher ökonomischer systeme sind und die keineswegs einer kapitalistischen Logik folgen. Paradoxerweise ist diese Arbeit wirtschaftspolitisch kaum anerkannt.

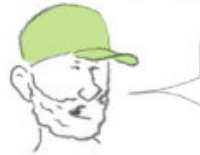

Es muss doch nicht die große Revolution stattfinden und wir müssen nicht aufirgendwelche zentralistische Maßnahmen warten. Und was auf Wasserversorgung und -verbrauch zutrifft, muss doch nicht in gleicher Weise auf andere Konsumbereiche zutreffen, oder?

Das stimmt, soziale Praktiken sind heterogen und das Beispiel mit dem Wasser lässt sich nicht pauschal auf andere alltägliche Handlungsfelder wie Ernährung, Mobilität, Heizen etc. übertragen. Es bleibt aber festzuhalten, dass durch individuelles verhalten Praktiken in einem sehr begrenzten Rahmen verändert werden können.

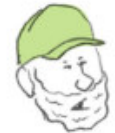

Nur durch Bottom-up-Initiativen der menschen, die nicht unbedingt ganze soziale Praktiken umkrempeln wollen, sondern einfach bei einem der Elemente einer sozialen Praxis ansetzen, ist es möglich, dass 2.B. eine solidarische Landwirtschaft oder etwas wie containern, Car-sharing entstehen, die eben versorgungsmöglichkeiten jenseits traditioneller Märkte etablieren.

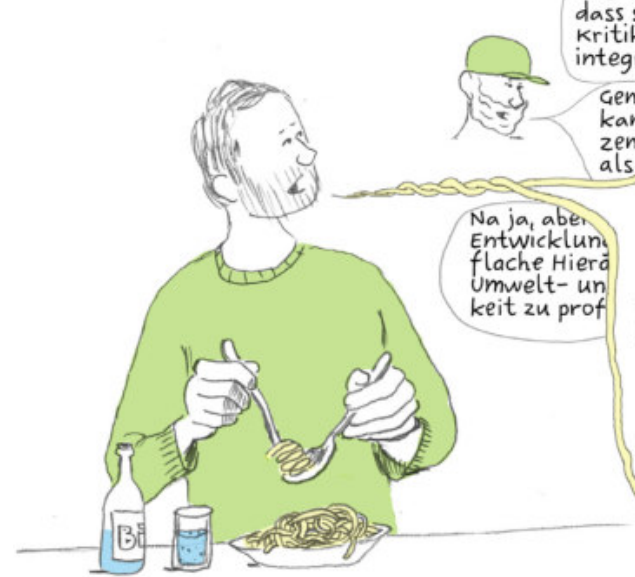

Das wäre schön, wäre da nur nicht die Erfahrung dass sich der Kapitalismus alles einverleibt, jegliche kritik und Gegenbewegungen in seine innere Logik integriert und damit erstarkt.

Genau das meinte ich: Mit dem kapitalozentrischen Blick kannst du in da. alan konsument *innen-produzent*;. aupt nichts anderes sehen als. Ich unterbreche nur ungern, aber es klingt nicht nach einer einfachen und eindeutigen Antwort auf die zweifel, die ich am Anfang hatte. Und inzwischen habe ich Hunger. Ich muss dringend etwas essen. Danach schreite ich konsumier.. äh, ich meine: 


\section{ZUM AUTOR}

Yen Sulmowski ist zur Zeit wissenschaftlicher Mitarbeiter am Wissenschaftlichen Zentrum Genealogie der Gegenwart an der Carl von Ossietzky Universität Oldenburg. $\mathrm{Zu}$ seinen Arbeitsschwerpunkten gehören praxissoziologische und ethnographische Forschung zu sozial-ökologischen Transformationen, Science and Technology Studies sowie graphisches Erzählen.

Der Beitrag wurde redaktionell von Andreas

Schulz betreut und von Tatiana Huppertz und Tamara Schwertel lektoriert.

\section{ENDNOTEN}

1. Jacobsen/Dulsrud 2007.

2. Zur Rolle von Verantwortung in Diskursen und Praktiken der gesellschaftlichen Transformation in Richtung Nachhaltigkeit s. Henkel/Lüdtke/Buschmann/Hochmann 2018.

3. Shove/Pantzar/Watson 2012; Alkemeyer/Buschmann/ Michaeler 2015; s. auch Brand 2011.

4. vgl. Shove 2003.

5. Praxistheorien gehen unterschiedlich mit der Eingrenzung einer zu beschreibenden sozialen Praxis um, so dass eine Bezeichnung eines Tätigkeitszusammenhangs als Praxis unterschiedlich ausfallen kann. Sobald der direkte situative Beobachtungsmoment überschritten wird, muss i.d.R. auf andere Methoden der Untersuchung und Beschreibung zurückgegriffen werden (z.B. Genealogie, Akteur-Netzwerk-Theorie, Diskursanalyse).

6. Shove/Pantzar/Watson 2012; s. auch Latour 2007.

7. Die Praxis des Körperwaschens war im 19. und bis Mitte des 20. Jahrhunderts in Nord- und Westeuropa viel stärker durch soziale Ungleichheit ausdifferenziert als dies heute der Fall ist. Die Darstellung dieser Praxis im vorliegenden Essay ist eine Art Schnittmenge der vielen damals parallel existierenden Praktiken des Körperwaschens. Siehe hierzu Dupke 1997, Frey 1997, Trupat 1996, Vigarello 1988.
8. Shove 2003, Vigarello 1988. Diese Diskurse waren zugleich Teil der Ideologien der Nationenbildung (Spieker 1996) und der Ausweitung staatlicher Macht- und Kontrollapparate (Frey 1997).

9. Gossmann 1996, vgl. auch Shove 2003.

10. Shove 2003.

11. Ottillinger 2011.

12. Lupton/Miller 1992.

13. Ottillinger 2011.

14. Bayerl 2003.

15. Statistisches Bundesamt 2015, Bayerl 2003.

16. Grunwald 2010, s. auch Fink 2012.

17. Brand 2011.

18. Sedlmaier 2018.

19. Brand/Wissen 2017.

20. Gibson-Graham 2014.

21. Habermann 2009, Brand/Wissen 2017; Gottschlich 2013.

22. Biesecker/Hofmeister 2006.

\section{LITERATUR}

Alkemeyer, Thomas/Buschmann, Nikolaus/Michaeler, Matthias (2015): Kritik der Praxis. In: Alkemeyer, Thomas/ Schürmann, Volker/Volbers, Jörg (Hrsg.) Praxis denken. Kritik und Konzepte. Wiesbaden: Springer, S. 25-50.

Bayerl, Günter (2003): Konsum, Komfort und Netzwerke. Die Versorgung mit Wasser. In: Reith, Reinhold/Meyer, Torsten (Hrsg.): Luxus und Konsum. Eine historische Annäherung. Münster: Waxmann, S. 129-158.

Biesecker, Adelheid/Hofmeister, Sabine (2006): Die Neuerfindung des Ökonomischen. Ein (re)produktionstheoretischer Beitrag zur sozial-ökologischen Forschung. München: Oekom.

Brand, Karl-Werner (2011): Umweltsoziologie und der praxistheoretische Zugang. In: Groß, Matthias (Hrsg.): Handbuch Umweltsoziologie. Wiesbaden: VS Verlag für Sozialwissenschaften, S. 173-198.

Brand, Ulrich/Wissen, Markus (2017): Imperiale Lebensweise. Zur Ausbeutung von Mensch und Natur im globalen Kapitalismus. München: Oekom. 
Dupke, Thomas (1997): Von der „Wasserkalamität“ zur „Wasserbequemlichkeit“. In: Wisotzky, Klaus/Zimmermann, Michael (Hrsg.): Selbstverständlichkeiten: Strom, Wasser, Gas und andere Versorgungseinrichtungen: die Vernetzung der Stadt um die Jahrhundertwende. Essen: Klartext, S. 10-45.

Fink, Pierre-Christian (2012): Wasserversorgung: Schluss mit dem Wassersparen!. In: Die Zeit, 29. März 2012. Online verfügbar unter, https://www.zeit.de/2012/14/Wasserversorgung (08.05.2018).

Frey, Manuel (1997): Der reinliche Bürger: Entstehung und Verbreitung bürgerlicher Tugenden in Deutschland; 1760 1860. Göttingen: Vandenhoeck \& Ruprecht.

Gibson-Graham, J. K. (2014): Rethinking the Economy with Thick Description and Weak Theory. In: Current Anthropology Jg. 55/9, S. 147-153.

Gossmann, Ulla (1996): „So viel Unheil quillet aus dem schmutzigen Unterrocke!“. Ratschläge zur Körper- und Schönheitspflege im „hygienischen“ 19. Jahrhundert. In: Löneke, Regina/ Spieker, Ira (Hrsg.): Reinliche Leiber schmutzige Geschäfte: Körperhygiene und Reinlichkeitsvorstellungen in zwei Jahrhunderten. Göttingen: Wallstein, S. $87-112$.

Gottschlich, Daniela (2013): Doing away with "labour": working and caring in a world of commons. Expeditions into (re)thinking the role of human (re)productive activity and its inherent nature in a generative commons network. Keynote Speech at the Economics and the Commons Conference 2013. Online verfügbar unter https://www.boell. de/sites/default/files/assets/boell.de/images/download_ de/201305_Keynote_Gottschlich_Working_and_Caring pdf (12.06.2018).

Grunwald, Armin (2010): Die Ökologie der Individuen. Erwartungen an individuelles Umwelthandeln. In: Büscher, Christian/Japp, Klaus Peter (Hrsg.): Ökologische Aufklärung. Wiesbaden: VS Verlag für Sozialwissenschaften, S. 231-257.

Habermann, Friederike (2009): Halbinseln gegen den Strom: Anders leben und wirtschaften im Alltag. Königstein/ Taunus: Helmer.

Henkel, Anna/Lüdtke, Nico/Buschmann, Nikolaus/Hochmann, Lars (Hrsg.) (2018): Reflexive Responsibilisierung. Verantwortung für nachhaltige Entwicklung. Bielefeld: Transcript.
Jacobsen, Eivind/Dulsrud, Arne (2007): Will Consumers Save The World? The Framing of Political Consumerism. In: Journal of Agricultural and Environmental Ethics, Jg. 20/5, S. 469-482.

Latour, Bruno (2007): Eine neue Soziologie für eine neue Gesellschaft. Einführung in die Akteur-Netzwerk-Theorie. Frankfurt am Main: Suhrkamp.

Lupton, Ellen/Miller, J. Abbott (Hrsg.) (1992): The bathroom, the kitchen, and the aesthetics of waste: a process of elimination; [publ. in conjunction with the Exhibition The Process of Elimination: the Kitchen, the Bathroom, and the Aesthetics of Waste, MIT List Visual Arts Center, May 9-June 28, 1992]. MIT List Visual Arts Center.

Ottillinger, Eva B. (Hrsg.) (2011): Intime Zeugen: Vom Waschtisch zum Badezimmer; [das Buch erscheint zur Ausstellung „Intime Zeugen. Vom Waschtisch zum Badezimmer" im Hofmobilendepot, Möbelmuseum Wien, 21. September 2011 - 22. Jänner 2012]. Wien: Böhlau.

Sedlmaier, Alexander (2018): Konsum und Gewalt: Radikaler Protest in der Bundesrepublik. Berlin: Suhrkamp.

Shove, Elizabeth (2003): Comfort, cleanliness and convenience. The social organization of normality. Oxford [u.a.]: Berg.

Shove, Elizabeth/Pantzar, Mika/Watson, Matt (2012): The dynamics of social practice: everyday life and how it changes. Los Angeles [u.a.]: Sage.

Spieker, Ira (1996): ,Jedem Deutschen wöchentlich ein Bad!“. Die Popularisierung von Volksbädern um die Jahrhundertwende und ihre Einrichtung im ländlichen Raum. In: Löneke, Regina/Spieker, Ira (Hrsg.): Reinliche Leiber - schmutzige Geschäfte: Körperhygiene und Reinlichkeitsvorstellungen in zwei Jahrhunderten. Wallstein: Göttingen, S. 113-140.

Statistisches Bundesamt (2015): Umwelt. Öffentliche Wasserversorgung und öffentliche Abwasserentsorgung. Fachserie 19, Reihe 2.1.1. Wiesbaden. Online verfügbar unter https://www.destatis.de/DE/Publikationen/Thematisch/UmweltstatistischeErhebungen/Wasserwirtschaft/ WasserOeffentlich.html (05.06.2018).

Trupat, Christin (1996): „Bade zu Hause!“ Zur Geschichte des Badezimmers in Deutschland seit der Mitte des 19. Jahrhunderts. In: Technikgeschichte, Jg. 63/3, S. 219-236. Vigarello, Georges (1988): Wasser und Seife, Puder und Parfüm: Geschichte der Körperhygiene seit dem Mittelalter. Frankfurt am Main: Campus. 


\section{geSIS}

\section{Sie forschen}
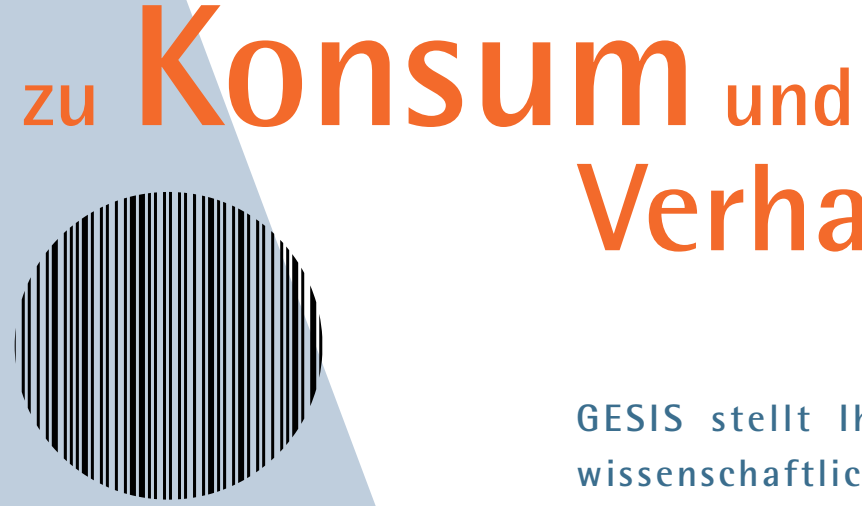

Verhalten?

GESIS stellt Ihnen aktuelle wissenschaftliche Methoden, qualitativ hochwertige Daten

Ihr Partner

für exzellente Forschung

www.gesis.org 


\title{
Führe mich sanft ans gleißend helle Licht
}

\author{
Konsequenzen des nudging für das \\ Konsument innenverhalten
}

Nudging ist seit knapp einer Dekade eine viel diskutierte Technik zur Beeinflussung des menschlichen Verhaltens. Angetreten mit der Ambition, das Leben der Menschen durch gezielte Entscheidungsarchitektur zu verbessern, findet es mittlerweile in unterschiedlichsten gesellschaftlichen Bereichen Anwendung. Um die Konsequenzen dieser Umkonfiguration des Sozialen identifizieren zu können, bedarf es Forschung. Indem eine Verbindung zwischen nudging und einer Statusverschiebung von Privatheit postuliert wird, versucht diese Arbeit dem Forschungsbedarf theoretisch beizukommen. Dazu wird das von Susan Leigh Star eingeführte Konzept des Grenzobjekts mit Michel Foucaults Überlegungen zur Gouvernementalität der Gegenwart verknüpft, um zu plausibilisieren, dass nudging unter gegenwärtigen gesellschaftlichen Verhältnissen traditionelle privatheitliche Normen destabilisiert. Deutlich gemacht wird das für den Bereich des Konsums, in dem die Datenökonomie als treibender Faktor diese Entwicklung fördert.

\section{Schlagwörter}

nudging; informationelle Privatheit; neoliberale Gouvernementalität; Grenzobjekt; datengetriebene Ökonomie; Verhaltensbeeinflussung 
Schaut man sich die Liste der Preisträger innen des Wirtschaftsnobelpreises der vergangenen 20 Jahre an, finden neben Beiträgen zu Spieltheorie, Marktdesign oder Finanzökonomie immer häufiger verhaltensökonomische Arbeiten Anerkennung. Zu nennen sind hier vor allem Daniel Kahneman, der unter anderem für seine Arbeiten zur Fehlbarkeit menschlichen Verhaltens 2002 ausgezeichnet wurde sowie Richard $\mathrm{H}$. Thaler, dem vergangenes Jahr für seine Beiträge zur Beeinflussung menschlichen Verhaltens die Ehre zuteilwurde. Während beide als Zweifler des spätestens seit Beginn der Finanzkrise 2007 immer mehr in die Kritik geratenen homo oeconomicus-Modells auftreten, verspricht insbesondere Thaler einen Weg gefunden zu haben, der begrenzten Rationalitätsbegabtheit von Marktakteur_innen angemessen Rechnung tragen zu können. Sein Zauberwort dafür lautet nudging. Ein unter diesem Titel gemeinsam mit dem Rechtswissenschaftler Cass R. Sunstein verfasstes Buch erschien erstmals 2008 und bietet seitdem Anlass für lebendige, vielfältige und vor allem resonanzstarke Diskussionen rund um das Thema nudging.

Der Anspruch des vorliegenden Beitrags ist es nachzuverfolgen, welche Konsequenzen nudging für den Bereich des Konsums mit sich bringt und welche Probleme dabei auftauchen. Es mangelt, so die hier vertretene These, dem von Thaler und Sunstein

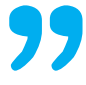

Welche Probleme können durch eine nudging-orientierte Adressierung der Konsument_innen entstehen?

vorgeschlagenen Konzept an Sensibilität für strukturelle Veränderungsdynamiken, die mit einer Implementierung von nudges in konsumbezogene Kontexte verbunden sind. Dies liegt daran, dass sie den Blick auf das Verhältnis zwischen nudgers (diejenigen, die nudges entwerfen; von ihnen choice architects genannt) und nudgees (diejenigen, die genudged werden) legen. Dadurch entgehen ihnen mögliche nicht-intendierte infrastrukturelle Folgen, die mit einem solchen Strukturwandel - gemeint ist in dieser Arbeit vor allem der sich verändernde Status der informationellen Privatheit - einhergehen. Die Idee des nudging-Ansatzes, die Konsument_innen zu besseren Entscheidungen zu bewegen, beinhaltet also auch immer implizite Normvorstellungen, die den Entscheidungsarchitekturen eingeschrieben sind. Damit verbunden lässt sich die Frage formulieren, ob die Entscheidungen tatsächlich besser sind und wenn ja warum und für wen. 
Diese stichwortartigen Überlegungen münden in die folgende Fragestellung: Welche Probleme können durch eine nudging-orientierte Adressierung der Konsument_innen entstehen?

Zur Beantwortung dieser Fragestellung wird in einem ersten Schritt das nudging-Konzept von Thaler und Sunstein eingeführt. Im Anschluss daran wird eine kurze Rekapitulation der bisher geäußerten Kritik an dem Konzept vorgestellt. Als Ergänzung zu dieser Kritik wird das für diese Arbeit zentrale Instrumentarium dargestellt, das eine bislang eher vernachlässigte Perspektive auf die blinden Flecken des nudging-Konzepts einzunehmen verspricht. Bezugsquellen sind hier die Arbeiten von Susan Leigh Star (2017) zum Thema des Grenzobjekts sowie Michel Foucaults (2004) Vorlesungen zur Gouvernementalität der Gegenwart. Beide Ansätze sollen zunächst gesondert voneinander mit nudging ins Gespräch gebracht werden. In einem letzten Schritt werden beide Perspektiven mit dem Ziel gebündelt, aufzuzeigen, dass nudging gerade im Bereich des Konsums eine große Bedrohung privatheitlicher Standards informationeller Natur darstellt. Im Fazit werden die gewonnenen Ergebnisse zusammengefasst und mögliche Leerstellen der Arbeit diskutiert.

\section{Nudging - Regieren mit lockerer Hand}

Was ist mit nudging gemeint? Thaler und Sunstein zufolge leitet es sich von dem Wort to nudge ab, was so viel bedeutet wie anstupsen oder anstoßen (im Original ,to push mildly or poke gently"; Thaler/Sunstein 2009: 4). Im verhaltensökonomischen Zusammenhang ist damit gemeint, den nudgees zwar ein bestimmtes Verhalten nahezulegen, es aber auch nicht zu erzwingen. Ziel jedes nudges sollte dabei sein, „to move people in directions that will make their lives better" (Thaler/Sunstein 2009: 6). Für diese Art der Verhaltenssteuerung prägten sie bereits in einem früheren Aufsatz das auf den ersten Blick paradox erscheinende Begriffspaar „libertarian paternalism" (Thaler/Sunstein 2003: 175). In ihren Augen vereint es die beiden zentralen Elemente des nudging: Zum einen soll der libertäre Aspekt dadurch gesichert werden, dass die Individuen "free to choose“ (Thaler/Sunstein 2009: 5) sind, wenn sie genudged werden. Es soll ein Verhalten nahelegt werden, mehr nicht. Wie entschieden wird, liegt demzufolge nach wie vor bei den Individuen; das beinhaltet auch, den nudge zu ignorieren bzw. dessen Vorschlag abzulehnen (von Thaler und Sunstein als opt-out Option bezeichnet; vgl. ebd.: 5). Die paternalistische Komponente wiederum besteht in dem bereits angesprochenen Anspruch, das Leben der Individuen durch nudges zu verbessern. Dieses Ziel basiert 
auf zwei Grundannahmen: Erstens die Anzweiflung des homo oeconomicus als realistisches Akteur_innenmodell und zweitens die Natur von Entscheidungen in einer multioptionalen Gesellschaft, die mitunter so komplex ist, dass ein fehlbares Individuum ohne Expert_innenwissen mit hoher Wahrscheinlichkeit nicht dazu in der Lage ist, ein im eigenen Interesse bestes Ergebnis zu erzielen (vgl. Thaler/ Sunstein 2009: 19ff.).

Bei der Implementierung von nudges kommt den sogenannten choice architects eine zentrale Rolle zu. Sie designen nudges. Ihre Aufgabe ist es, das Verhalten, das von einem nudge getriggert werden soll, so intuitiv und selbstverständlich wie möglich erscheinen zu lassen (vgl. Thaler/ Sunstein 2009: 89f.). Um dies zu gewährleisten, müssen nudges bzw. deren Design grundlegenden Prinzipien menschlicher Psychologie entsprechen (vgl. Thaler/Sunstein 2009: 90).

Neben den eher grundsätzlich gehaltenen Ratschlägen liefern Thaler und Sunstein auch konkrete Vorschläge - sechs an der Zahl - zur Modellierung von nudges (vgl. Thaler/Sunstein 2009: 93-109). Ohne auf alle sechs Nudgingdesigns genauer einzugehen, kann festgehalten werden, dass sie miteinander kombinierbar sind und je nach Entscheidung auf ihre Effizienz überprüft werden müssen. Das entscheidende Merkmal, das sie voneinander unterscheidet, liegt in der Art und Weise, wie nudgees adressiert werden. Mal fällt das subtil aus, wie bei der default option-Variante (als prominentes Beispiel sei hier die default option bei Druckern erwähnt, beidseitig zu drucken), mal offensiver, wie bei der expect error-Variante (visuelle Parkhilfen bei Fahrzeugen können hier als Beispiel genannt werden) (vgl. Thaler/Sunstein 2009: 95-99). Bei dem ersten Beispiel müssen die nudgees nichts tun, ihnen wird die Entscheidung abgenommen, beidseitig $\mathrm{zu}$ drucken, da es voreingestellt ist. Im zweiten Fall leitet der nudge sie an, aktiv zu werden, also zu bremsen bzw. umstehende Autos oder ähnliches nicht zu rammen. Hier wird also ein aktives Handeln der nudgees gefordert. Diese Beispiele verdeutlichen, dass sich für choice architects immer die Frage danach stellt, ob es eher sinnvoll ist, einen nudge transparent oder intransparent zu gestalten bzw. aktives Handeln der Individuen einzufordern oder es ihnen abzunehmen. Je nach Situation und Entscheidungsdringlichkeit fällt die Antwort unterschiedlich aus (vgl. Hansen/ Jespersen 2013: 23-27).

Dass die beiden Autoren mit ihrem Konzept einen Nerv der Zeit getroffen haben und eine hohe Nachfrage nach ihrer Expertise erzeugen konnten, kann nicht nur daran abgelesen werden, dass Sunstein von 2009 bis 2012 Leiter des Office of Information and Regulatory Affairs, einer wichtigen Regulationsbehörde in den USA, 
war (vgl. Broder 2012). Auch die wissenschaftliche Debatte ist äußerst lebendig. Unterschiedliche Disziplinen arbeiten sich am nudging ab, es gibt Themenschwerpunkte (Law \& Policy 2016; Review of Philosophy and Psychology 2015) und Tagungen zu dem Thema (zuletzt fand die Tagung Nudging and Information 2018 Anfang März diesen Jahres in Graz statt).

Was den Bereich des Konsums betrifft, wird nudging eine hohe Chance zugeschrieben, Verhaltensroutinen zu verändern. So sehen beispielsweise Thorun et al. in einer von dem Umweltbundesamt beauftragten Studie die Stärke von nudges darin begründet, „dass Verhaltensänderungen erreicht werden können, ohne dass die Wahlfreiheit von Verbraucherinnen und Verbrauchern eingeschränkt wird“ (Thorun et al. 2016: 82). Eine Notwendigkeit zur Verhaltensänderung untersuchen die Autor_innen in diesem Zusammenhang anhand von Konsummustern, die einer ökologischen Nachhaltigkeitsmaxime nicht gerecht werden. Ihr Schlussfolgerungen legen nahe, dass nudges dabei helfen können, einen nachhaltigeren Konsum bei geringen monetären Kosten zu erreichen (vgl. Thorun et al. 2016: 67).

\section{Nudging und seine Kritiker_innen}

Thaler und Sunsteins Vorschlag blieben nicht kritiklos. Die für diesen Beitrag vier wesentlichen Kritikpunkte sind: 1 . Nudging sei manipulativ; 2 . Nudging mangele es an Effizienz; 3. Die Rolle der choice architects müsse reguliert werden und 4. Nudging gefährde die informationelle Privatheit der adressierten Individuen.

Am häufigsten wird beanstandet, dass nudging manipulativ sei. Ausgangspunkt für die Kritik ist das von Thaler und Sunstein verwendete Begriffspaar libertarian paternalism. Während ihnen zufolge nudges imstande sind, diese eigentlich konträren Begriffe miteinander zu versöhnen, verneint die Kritik dieses Potential. T. M. Wilkinson beispielsweise stuft nudging deshalb als manipulativ ein, weil es die begrenzte Rationalität der Individuen ausnutze:

Is it not manipulation to take advantage of people's predictable deviations from economic rationality? And if it is manipulation, how can the nudging be libertarian? (Wilkinson 2013: 342)

Stefan Piasecki wird noch grundsätzlicher, wenn er schreibt, dass „bereits die Planung von Handlungsoptionen eine Manipulation des Willens von Menschen dar[stelle]“ (Piasecki 2017). Auch die für Thaler und 
Sunstein wichtige Option des opt-out reiche nicht, sie vom Manipulationsvorwurf freizusprechen (vgl. Wilkinson 2013:354).

Ergänzt wird diese Kritik am libertären Anspruch des nudging durch die Infragestellung des paternalistischen Aspekts. Es finden sich Stimmen, die zum einen generell die Effizienz von nudges anzweifeln (vgl. Beck 2018: 224), und andere, die einen Fehler darin sehen, dass nudging Individuen und nicht Kollektive adressiert. Vor allem gehe es darum, die Gesellschaft als Ganze zum Besseren zu bewegen, nicht nur die Wohlfahrt einzelner Individuen (vgl. Goodwin 2012: 90; Högy 2014: 32). Und dazu seien nudges nicht in der Lage:

Nudging is unable to deliver the kind of substantive changes that are needed to tackle the big problems that society faces. (Goodwin 2012: 89f.)

Ein Grund dafür liege darin, dass nudges den komplexen globalen Problemen nicht angemessen Rechnung tragen könnten (vgl. Ekardt 2017). Stattdessen könne „gesellschaftlicher Wandel nur im Wechselspiel der verschiedenen voneinander abhängigen Akteure“ (Ekardt 2017) gelingen. Einzelne Individuen $\mathrm{zu}$ adressieren, um gesamtgesellschaftliche Probleme zu bewältigen, erscheint aus dieser Perspektive sowohl ökonomisch als auch ideologisch als der falsche Weg.
Ein weiterer Kritikpunkt betrifft die Rolle des choice architects. Neben der Einschätzung, dass er_sie dafür die Verantwortung trägt, ob ein nudge manipulativ ist oder nicht (vgl. Wilkinson 2013: 354), finden sich zudem Fragen dazu, wie die normative Ausgestaltung einer solchen Position auszusehen hat. Treffend formuliert der Ökonom Hanno Beck: „Welche Ziele, welche Werte sollen mit dem nudging eigentlich genau verfolgen [sic!] werden?“ (Beck 2018: 225) Rechtlich als auch gesellschaftlich wurde noch keine Einigung erzielt, in welchen lebensweltlichen Bereichen und in welchem Maße Individuen genudged werden dürfen oder sollen (vgl. Högy 2014: 33; Piasecki 2017; Thorun et al. 2016: 61). Auch die Tatsache, dass für nudgees die choice architects häufig nicht identifizierbar sind, wird als Begründung dafür angeführt, eine Kontrollinstanz zu fordern, um so einem möglichen Missbrauch entgegenzutreten (vgl. Högy 2014: 33; Piasecki 2017). Die Gefahr, die hier gesehen wird, liegt in der Intransparenz der Intentionen der choice architects einerseits und dem potentiellen Einfluss, den sie ausüben, andererseits (vgl. dazu auch Thaler/Sunstein 2009: 238-241).

Der letzte Kritikpunkt betrifft das von den beiden Autor_innen Andreas Kapsner und Barbara Sandfuchs thematisierte Verhältnis von nudging und informationeller Privatheit. Mit Bezug auf Beate Rössler verstehen sie unter informationeller Privatheit folgendes: Sie hat dann Bestand, wenn 
persönliche Informationen von Individuen „vor [...] unerwünschten Zugriffen anderer" (Rössler 2001: 23; vgl. Kapsner/ Sandfuchs 2015: 458f.) geschützt sind. Die Kontrolle und Verfügungsgewalt persönlicher Daten muss demnach bei jedem Einzelnen selbst liegen. Im folgenden Zitat wird deutlich, dass effizientes nudging dieses Gebot zwangsläufig unterläuft:

The more one knows about people's preferences, past choices, social connections, daily routines, and so on, the easier it is to nudge them successfully, since many types of nudges can be more precisely targeted on the basis of such information. (Kapsner/Sandfuchs 2015: 456)

Ihr Argument ist kausaler Natur: Je mehr über Personen bekannt ist, umso besser lassen sie sich nudgen. Diese Kausalbeziehung sorgt dafür, dass dem Sammeln möglichst umfangreicher, personenenbezogener Daten eine schlüssige und nachvollziehbare Begründungslogik unterstellt werden kann. Kapsner und Sandfuchs sehen darin eine große Gefahr für die informationelle Privatheit von Individuen und fordern, dass bei der Planung von nudges immer die potentiellen, privatheitsbezogenen Konsequenzen mitbedacht werden müssten (vgl. Kapsner/ Sandfuchs 2015: 467). Es gehe letztlich um eine Ausbalancierung zwischen der Gewährleistung individueller Privatheit und den Zielen, die mit dem nudging verbunden werden (vgl. Kapsner/Sandfuchs 2015: 465).

\section{Der Mittelweg zwischen sub- jektivistischer und objektivis- tischer Kritik: Die Perspektive der Infrastruktur}

Der Vorschlag, den diese Arbeit nun als Ergänzung zu den aufgezählten Kritikpunkten unterbreiten möchte, liegt darin, nudging weder aus einer subjektivistischen noch einer objektivistischen Perspektive zu betrachten, sondern auf das Dazwischen - hier mit dem Begriff Infrastruktur gekennzeichnet - zu fokussieren. Die subjektivistische Perspektive einerseits zeichnet sich dadurch aus, dass nudging Kritik erfährt, weil Individuen manipuliert werden. Wenn die Rolle der choice architects kritisiert wird, dann deshalb, weil ihre Motivationen und Ziele den Individuen intransparent bleiben. Wenn nudging vorgeworfen wird, privatheitliche Konsequenzen nicht mitzudenken, dann deshalb, weil der Status der individuellen Privatheit infrage steht. Andererseits finden sich objektivistische Positionen: Im Namen des Klimaschutzes, im Namen der Gesellschaft, im Namen der Konsumkritik etc. werden auf die Unzulänglichkeiten/ die mangelnde Effizienz des nudging hingewiesen. Nudging ist in dieser Hinsicht deshalb nicht überzeugend, weil es große, objektive Probleme nicht zufriedenstellend lösen kann, so die Kritik. 


\section{Mittelweg Nr. 1: Nudging hat Erfolg, weil es als Konzept schlecht strukturiert ist}

Was ist nun mit einem (infra-)strukturellen Blick gewonnen? Aus meiner Perspektive kann der Diskurs um nudging mithilfe des (infra-)strukturellen Blicks auf zwei Ebenen erhellt werden. Diese finden in Form von Thesen ihren Ausdruck.

Eine große, vermeintliche Schwäche des Buches von Thaler und Sunstein liegt darin, Ausarbeitung des nudging-Konzepts vorzufinden ist. Die wenigen generalisierenden Aussagen, die sie treffen - beispielsweise die Abgrenzung zum homo oeconomicusModell - tragen zwar zum Verständnis ihres Anspruches bei. Doch bleibt ihr Konzept selbst sehr vage. Das kann unter anderem daran abgelesen werden, dass ihr Buch beinahe ausschließlich mit Beispielen argumentiert und theoretische Diskussionen so gut es geht vermeidet. Gerade diese theoretische Vagheit kann als wichtiger Faktor für den Erfolg des nudging gewertet werden. Diese vielleicht auf den ersten Blick kontraintuitive Aussage, dass seine Vagheit den Erfolg des nudging massiv unterstützt, wird nachvollziehbar, wenn man Susan Leigh Stars Idee des Grenzobjekts heranzieht. Daher folgende 1. These: Das Konzept des nudging ist deshalb so erfolgreich, weil es als Grenzobjekt zur Kooperation unterschiedlicher Akteur_innen einlädt.

Leigh Star, eine vor allem in den Science and Technology Studies einflussreiche Autorin, weist in einer von ihr und James R. Griesemer durchgeführten Studie zur Entstehung des Museum of Vertebrate Zoology in Berkeley darauf hin, dass es Grenzobjekte sind, welche die Kooperation von Akteur_innen mit unterschiedlichen, auch divergierenden Interessen ermöglichen (vgl. Leigh Star/Griesemer 2017). Im Fall des Naturkundemuseums listet Leigh Star den Direktor des Museums, Joseph Grinnell, Annie Montage Alexander, eine Mäzenatin und Amateurnaturforscherin, Hobbysammler_innen, Fallensteller_innen sowie die Universitätsverwaltung der University of California als relevante Akteur innen auf (vgl. Leigh Star/Griesemer 2017: 92-100). Jede dieser Gruppen bzw. Einzelpersonen verfolgen eigene Visionen. Grinnell geht es beispielsweise um eine möglichst akkurate und nachvollziehbare Form des Sammelns und Kategorisierens der Ausstellungsobjekte, während für die Fallensteller_innen das Geschäft mit erlegten Tieren im Vordergrund steht. Diese unterschiedlichen Interessen so zu versöhnen, dass alle Beteiligten sowohl ihren eigenen Interessen nachgehen als auch einem übergeordneten Ziel dienen können, liegt in der Macht der Grenzobjekte (vgl. Leigh Star/Griesemer 2017: 105).

Was im Feld als Grenzobjekt auftritt, lässt sich nie a priori festlegen (vgl. Leigh Star 2017: 224f.). Sie - und das spricht für 
Leigh Stars starke empirische Orientierung - votiert für einen offenen Begriff, dessen Bewandtnis erst in der Praxis erkennbar wird. Sind Grenzobjekte einmal am Wirken, eint sie deren Funktion, vielfältige Kooperation heterogener Gruppen zu ermöglichen, indem sie es erlauben, unterschiedliche Zielorientierungen ohne die Notwendigkeit eines Konsenses in Einklang zu bringen (vgl. Leigh Star/Griesemer 2017: 82). Dementsprechend finden sich Grenzobjekte vor allem in arbeitsteiligen Organisationen wie Unternehmen, Krankenhäusern, Laboren oder eben Museen.

Betrachtet man im Anschluss an diese Überlegungen die hohe Nachfrage nach nudging und stellt sich die Frage, warum es einen derartigen Nachfrageschub erfahren hat, fällt die Antwort leicht: Nudging - und damit ist hier explizit das Konzept gemeint - ist deshalb so beliebt, weil es als Grenzobjekt verstanden wird. Während nudges immer eine Verhaltensbeeinflussung von Individuen und damit eine uni-direktionale Relation herstellen, ist im nudging-Konzept die Möglichkeit angelegt, eine Praxisgemeinschaft zu konstituieren und kollektiv Ziele zu verfolgen. Einen wichtigen Anhaltspunkt dafür, nudging als Grenzobjekt zu deuten, findet sich im
Buch von Thaler und Sunstein. Wenn sie sagen, dass es ihnen darum geht, „to move people in directions that will make their lives better" (Thaler/Sunstein 2009: 6), und dieses better letztlich unscharf bleibt, schaffen sie eine Unmenge an Anknüpfungspunkten, die auf je eigene Art konkretisiert werden können. Nudging forciert also das Zusammenarbeiten unterschiedlichster Personen. Auch wenn diese Aussage noch einer empirischen Untersuchung zur Untermauerung bedarf, scheinen Nachrichtenmeldungen über ,wirksames Regieren“ dies zumindest nahezulegen (vgl. Bundesregierung.de 2017). Es wird ein Gespräch gesucht, das unterschiedliche Gruppen zusammenbringen soll, um ein bestmögliches Ergebnis - einen bestmöglichen nudge - zu entwickeln. Die Arbeitsweise wird dabei als wissenschaftlich, interdisziplinär und partnerschaftlich charakterisiert (vgl. Bundesregierung.de 2017).

Ein weiterer Grund, der die These festigt, dass nudging als Grenzobjekt verstehbar ist, liegt in dem Begriffspaar libertärpaternalistisch. Während sich die übrige Kritik vor allem daran stört, dass die beiden Begriffe unvereinbar miteinander seien, also nudging weder richtig libertär noch richtig paternalistisch sei, kann mit Bezug auf Leigh Star festgestellt werden, 
dass es gar nicht darum geht, nudging als streng libertär oder streng paternalistisch zu entlarven. Die Chance, die in diesem Oxymoron liegt, besteht darin, nudging über Parteigrenzen, Links-Rechts-Einordnungen oder sonstigen, trennenden Maßstäben hinweg als zur Zusammenarbeit einladendes Tool zu verwenden. Es geht Leigh Star ja gerade darum, Grenzobjekte als etwas zu verstehen, das nicht auf einen homogenen und konsensuell befriedeten Arbeitszusammenhang angewiesen ist, sondern darum, dass Uneinigkeit und Heterogenität von Grenzobjekten getragen werden können (vgl. Leigh Star/Griesemer 2017: 86f.). Dazu passen auch die Ausführungen von Thaler und Sunstein, die ganz explizit in der Einleitung ihres Buches darauf hinweisen, dass Libertarismus und Paternalismus kein Gegensatz sein muss. Konkret beziehen sie sich auf die politische Landschaft in den USA, wenn sie schreiben: „In short, libertarian paternalism is neither left nor right, neither Democratic nor Republican." (Thaler/Sunstein 2009: 15) Sie verstehen ihr Anliegen vor allem als ein pragmatisches: „we are not for bigger government, just for better governance." (Thaler/Sunstein 2009: 15; Hervorhebung im Original) Ihnen geht es darum, das Regieren zu verbessern. Und zwar am besten so, dass auch und gerade unterschiedliche politische Lager Anknüpfungspunkte finden können.

\section{Mittelweg Nr. 2: Bekannte Men- schenführungstechnik in neuem Gewand}

Bezog sich die erste These noch auf die produktive, weil zur Kooperation anstiftende Kraft des nudging, schlägt die zweite einen im weitesten Sinne regierungskritischen Blick vor.

These 2: Nudging ist eine konsequente Exekution neoliberaler Regierungstechnik und bleibt damit einer ökonomistischen Sicht auf Gesellschaft verhaftet.

Die Frage, welche ethischen Standards mit nudging verbunden sind, ist eine große. Sunstein hat dazu in Reaktion auf Kritiken ein Paper veröffentlicht, in dem er das Konzept verteidigt (Sunstein 2015). Zwei Punkte sind ihm dabei besonders wichtig:

"First", both nudges and choice architecture are inevitable, and it is therefore pointless to wish them away. "Second", many nudges, and many forms of choice architecture, are defensible and even required on ethical grounds, whether we care about welfare, autonomy, dignity, or some other value. (Sunstein 2015: 415; Hervorhebung im Original)

Dass nudging bzw. choice architecture nicht zu vermeiden seien, haben beide Autoren bereits in ihrem gemeinsamen Buch betont (vgl. Thaler/Sunstein 2009: 
247). Doch scheint Sunstein nicht der Eindruck verlassen zu haben, dass dieser Punkt in der Rezeption zu wenig Beachtung gefunden hat und daher einer weiteren Betonung bedarf. Natürlich spielt er sich damit selbst in die Karten. Denn das Argument für gezieltes nudging lautet ja: Wenn schon beides - nudging und choice architecture - unvermeidbar ist, so sollte es doch am besten aktiv gestaltet werden, um so (politisch) definierte Ziele im Dienste der individuellen Wohlfahrt erreichen zu können. Gezielte nudges erhöhen demnach die Chance, den nudgees die Möglichkeit einzuräumen, eine gute, das heißt für sie richtige, Entscheidung treffen zu können. Ethische Fragen, so Sunstein, können erst dann Gegenstand einer Diskussion werden, wenn sie sich auf eine konkrete choice architecture beziehen. Abstrakte, also ohne ein konkretes empirisches Beispiel geführte Diskussionen hält er für überflüssig. So spricht er in diesem Zusammenhang etwa von einer "trap of abstraction“ (Sunstein 2015: 424).

Sunsteins Annahme, der zufolge es nicht sinnvoll ist, über nudging im Allgemeinen ethische Urteile zu fällen, verdeutlicht, wie sehr er sich gegenüber bestimmten politisch-philosophischen Sichtweisen verschließt. Doch gerade diese gewähren einen Blick auf nudging, der keinesfalls unterschlagen werden sollte. Sunstein kreiert ein Bild, in dem das unkontrollierte nudging ein potentielles Problem darstellt, das es mittels einer klug gewählten choice architecture zu bewältigen gilt. Dieser Bewältigungsversuch kann und muss jedoch auch unabhängig von empirischen Einzelbeispielen diskutierbar sein. Hier bietet es sich an, Michel Foucaults Überlegungen zur Gouvernementalität als konträre Position ins Feld zu führen. Foucault, der dem Thema Gouvernementalität zwei Vorlesungsreihen am Collège de France (1977-1978 und 1978-1979) widmete, versucht in diesen zu zeigen, inwiefern sich die Art und Weise der Regierung der Menschen seit dem 16. Jahrhundert entwickelt hat (vgl. Foucault 2004a, 2004b). Das Hauptaugenmerk liegt dabei darauf, wie und mit welchen Mitteln regiert wird. Also welche Rationalität, welche Taktiken, welche Kalküle im Regieren ihren Ausdruck finden. Ohne hier auch nur im Ansatz die Genealogie der Gouvernementalität, wie Foucault sie darstellt, wiederzugeben, soll die These im Folgenden plausibilisiert werden, warum nudging sehr gut mit einer spezifischen, nämlich der neoliberalen Gouvernementalität, vereinbar ist.

Für Foucault gilt nicht immer die Art des Regierens, wie sie heute zu beobachten ist, als dominant. Am Beispiel der überaus einflussreichen Schrift Der Fürst von Niccolò Machiavelli veranschaulicht er die Zielorientierung einer bestimmten Art des Regierungshandelns: „die Aufrechterhaltung [...] eines Fürstentums“ (Foucault 2004b: 149). Zur erfolgreichen 
Aufrechterhaltung formuliert Machiavelli ein Arsenal an Tugenden und Verhaltensweisen, die den Statuserhalt, ja eine Erweiterung des eigenen Territoriums versprechen. Bei ihm ist die Kunst des Regierens darauf bezogen, die eigene Macht, die eigene Souveränität abzusichern. Dieser Regierungsmodus ändert sich - und damit setzt für Foucault das gouvernementale Regieren ein - mit der Entdeckung der Bevölkerung als „höchste[m] Zweck der Regierung" (Foucault 2004b: 158). Im Vordergrund steht nicht mehr das möglichst geschickte und mitunter auch - je nach Erfordernis - verlogene und hinterhältige Tun des Fürsten zum eigenen Machterhalt, sondern eines zum Wohl der Bevölkerung:

Einen Staat zu regieren wird also heißen, die Ökonomie anzuwenden, eine Ökonomie auf der Ebene des Staates als Ganzem, das heißt, man wird, was die Einwohner, die Reichtümer, das Verhalten aller und jedes einzelnen betrifft, eine Form von Überwachung und Kontrolle ausüben [...]. (Foucault 2004b: 144)

Mit der Gouvernementalisierung des Staatshandelns tritt also die Frage auf, wie eine Bevölkerung möglichst ökonomisch zu regieren ist. Eine Verfahrensweise - und vielleicht die zeitgenössischste - ist die der neoliberalen Gouvernementalität. In Auseinandersetzung mit einflussreichen wirtschaftswissenschaftlichen Texten findet
Foucault zwei entscheidende Merkmale, die in seinen Augen das Neoliberale ausmachen. Erstens werden Verhaltensweisen, die bis dahin nicht in ökonomischen Kategorien gedacht wurden, nunmehr in solchen gedacht (Für Foucault gilt die Humankapitaltheorie, wie sie von Gary S. Becker eingeführt wurde, als gutes Beispiel; vgl. Foucault 2004a: 317-321). Zweitens gerät das Handeln der öffentlichen Gewalt unter ökonomische Aufsicht und wird mit Begriffen des Marktes ob seiner Effizienz kritisiert (vgl. Foucault 2004a: 342). Mit anderen Worten: Die neoliberale Gouvernementalität ist dadurch gekennzeichnet, dass ihre Regierungstechniken ökonomisch, d.h. nach wirtschaftswissenschaftlichen Kriterien operieren sollen. Das dazugehörige Akteur_innenmodell orientiert sich an dem des homo oeconomicus, was Foucault als „Unternehmer seiner selbst" (Foucault 2004a: 314) betitelt. Gemeint ist damit eine Bündelung vielerlei Funktionen auf eben dieses Subjektmodell, das „für sich selbst sein eigenes Kapital ist, sein eigener Produzent, seine eigene Einkommensquelle“(Foucault 2004a: 314). In den Vordergrund rückt die Bedürfnisbefriedigung des Einzelnen und damit verbunden die Anhäufung des je eigenen Humankapitals. 


\section{Ökonomische Rationalität bleibt Ökonomische Rationalität}

Zwei Argumente sollen nun die Einsicht stützen, dass nudging als neoliberale Regierungstechnik verstanden werden kann. Das erste Argument lautet, dass trotz Negierung des homo oeconomicus-Modells, wie es Thaler und Sunstein forcieren, das dahinterstehende Rationalitätsmodell weiterhin bestehen bleibt. Diese These arbeitet Ulrich Bröckling in seinem Aufsatz Nudging: Gesteigerte Tauglichkeit, vertiefte Unterwerfung heraus (vgl. Bröckling 2017: 184). Bröckling zufolge ist nudging überaus passfähig zum gängigen neoliberalen Gesellschaftsverständnis, da es die evidente Fehlbarkeit der Akteur_innen zu berücksichtigen vermag und gleichzeitig versucht, sie produktiv zu machen. In diesem Sinne lässt sich nudging als ein verstärkender Faktor für das Denken in ökonomistischen Kosten-Nutzen-Kalkulationen begreifen. Bröckling resümiert:

Der libertäre Paternalismus markiert [...] keinen Bruch mit der neoliberalen Anrufung unternehmerischer Tugenden. Er ergänzt vielmehr die konsequente Vermarktlichung des Selbst, indem er dessen konstitutive Rationalitätsschwächen zu kompensieren verspricht und ihm so dazu verhelfen will, seine ureigenen Interessen $z u$ verfolgen und rationalere Entscheidungen zu treffen. (Bröckling 2017: 188)
Nudging bleibt also in seiner Art der Menschenführung der Rationalität neoliberaler Gouvernementalität treu. Es ändern sich lediglich die Vorzeichen: Der homo oeconomicus von Thaler und Sunstein ist nicht von Natur aus rationalitätsbegabt, sondern bedarf der gezielten Nachhilfe durch nudges. Was infrage gestellt wird, sind die Fähigkeiten der Menschen, nicht die neoliberale Rationalität, also die „Rationalitätsnorm der Kosten-NutzenMaximierung" (Bröckling 2017: 184).

Das zweite Argument betrifft die von Thaler und Sunstein angenommene überzeitliche Präsenz von nudges. Indem sie behaupten, dass sie schon immer eine Rolle gespielt hätten, jedoch bei unbedachtem Einsatz dazu tendierten, gesellschaftlich nicht erwünschte Folgen zu produzieren, machen sie ihr Argument für ein gelenktes nudging stark. Mit Foucault lässt sich nun entgegnen, dass nudging erst dadurch zu einer Regierungstechnik werden konnte, indem „das Interesse als Interesse der Bevölkerung“ zur „Zielscheibe und [...] Hauptinstrument der Regierung " (Foucault 2004b: 158f.) wurde. Es muss erst ein Interesse daran bestehen, etwas Abstraktes wie die Erhöhung des Gemeinwohls zu adressieren, um nudging ins Arsenal der Regierungstechniken aufnehmen zu können. Dass es als neoliberale Regierungstechnik verstanden werden kann, liegt vor allem an seinem Rationalitätsdiktum: Es geht darum, „Wahlhandlungen [Einzelner; 


\section{[N] udges können nicht nur beratend wirken, sondern auch Nachfragewünsche evozieren, sie haben also eine produktive Komponente.}

E.H.] zu rationalisieren“ (Bröckling 2017: 190). Diese Programmierung der Subjektivität ist stets gerichtet auf die Bedürfnisbefriedigung einzelner Personen. Folge davon ist, wie Bröckling es beschreibt, dass nudging sich als „Entpolitisierung im Zeichen eines verallgemeinerten Behaviorismus [erweist], der demokratische Aufklärung durch Verhaltensmodifikation ersetzt.“ (Bröckling 2017: 191) In diesem Sinne lässt sich nudging als politisches Instrument zur Entpolitisierung begreifen, das gerade im Rahmen der neoliberalen Gouvernementalität ein hohes Maß an Überzeugungskraft für sich beanspruchen kann, da es verspricht, auch bei komplexen Gemengelagen die beste, das heißt nutzenmaximierende Entscheidungshilfe parat zu haben.

\section{Und was hat das jetzt mit Konsum zu tun?}

Versucht man nun die beiden Thesen zusammenzuführen und auf das Thema Konsum zu beziehen, lässt sich die Vergrößerung einer ohnehin schon großen Wissensasymmetrie festhalten: die zwischen Entscheidungsarchitekt_innen und

Konsument_innen. Nudging-Konzepte geraten dank interdisziplinären Arbeitsgemeinschaften immer ausgefeilter und komplexer, was eine Reflexion über diese immer schwerer macht. Welche normativen Erwartungen, welche Gestaltungsvorhaben mit nudges verbunden sind, bleibt unter dem Mantel der (vermeintlichen) individuellen Nutzenmaximierung verdeckt, bzw. stellt sich die Frage, welcher Nutzen welches/r Akteur_in maximiert wird. Denn: „Anstupsen können alle.“ (Bröckling 2017: 192)

Im Bereich des Konsums wird ein immenser Aufwand betrieben, möglichst hochfrequentiert und voraussagensicher Konsumentscheidungen einzuleiten. Um die Tragweite dieses Antriebs zu verstehen, lohnt es sich, die nudging-alsGrenzobjekt-These mit dem Kritikpunkt, den Kapsner und Sandfuchs formuliert haben, zusammenbringen. Denn neben der Versammlung von unterschiedlichen Expert_innen, um ein nudge zu konzeptualisieren, gehört als ebenso relevantes Tool der Algorithmus zu einer modernen Entscheidungsarchitektur. Dass in diesem Fall nudges nicht primär den Nutzen der Konsument_innen im Blick haben, ist beinahe selbstevident (vgl. Bröckling 
2017: 193). Dieses Spannungsverhältnis vor Augen, argumentieren Kapsner und Sandfuchs, dass der Existenzmodus von Algorithmen, immer mehr Informationen zu sammeln, um nudges zu optimieren, privatheitliche Normen als hinderlich betrachtet. Besonders interessant zu beobachten, ist diese Logik im Bereich des Konsums. Da personalisierte nudges in der Lage sind, den Erwerb von Konsumprodukten überzeugend zu plausibilisieren, liegt in ihnen zudem eine systemstabilisierende Funktion zugrunde: Sie können dafür sorgen, dass die Konsumökonomie nicht nur aufrechterhalten wird, sondern auch floriert. Denn nudges - und das darf im Zusammenhang einer datengetriebenen Ökonomie nicht vergessen werden - können nicht nur beratend wirken, sondern auch Nachfragewünsche evozieren, sie haben also eine produktive Komponente. Als gängiges Beispiel dafür kann die Rubrik „Kunden, die diesen Artikel kauften, kauften auch" auf der Website des Onlinehändlers Amazon gelten.

Wenn es also stimmt, dass nudging eine passfähige Regierungstechnik der neoliberalen Rationalität darstellt, wäre das
Gewicht, das auf die informationelle Privatheit drückt, ungleich höher. Dann wäre die These, dass sie nicht nur im Rahmen von datengetriebenen Ökonomien einen schweren Stand hat, sondern dass sie aus rationalen Gründen ein Hindernis für die Nutzenmaximierung der Unternehmer innen ihrer selbst darstellt. Ist man gewillt, aus dieser Perspektive Kritik im Sinne Foucaults zu üben - „die Kunst nicht dermaßen regiert zu werden“ (Foucault 1992: 12) - könnte es das dann heißen: Für den Wert der informationellen Privatheit verstanden als Voraussetzung individueller Autonomie (vgl. Rössler 2001: 26) - einzutreten, wäre gleichbedeutend damit, nicht mit der gegenwärtigen neoliberalen Gouvernementalität einverstanden zu sein.

\section{Fazit: Ist in der Welt des Kon- sums Platz für die Privatheit?}

Ausgangspunkt der Arbeit war die Fragestellung, welche strukturellen Problemlagen durch eine nudging-orientierte Adressierung der Konsument_innen entstehen können. Um zur Beantwortung dieser Frage zu gelangen, wurden in einem

\section{Für den Wert der informationellen Privatheit [...] einzutreten, wäre gleichbedeutend damit, nicht mit der gegenwärtigen neoliberalen Gouvernementalität einverstanden zu sein.}


ersten Schritt die wichtigsten Charakteristika des nudging herausgearbeitet. Im Anschluss daran fanden gängige, das heißt häufig anzutreffende Kritikpunkte an dem Konzept ihren Platz. Mit dem Anspruch, diesen Kritiken weitere, eher unterrepräsentierte Positionen an die Seite zu stellen, wurden zwei Thesen aufgestellt, die eine (infra-) strukturelle Perspektive bieten: 1 . Nudging als Konzept ist ein Grenzobjekt. 2. Nudging fungiert als neoliberale Regierungstechnik. Im Versuch, die beiden Thesen zu konvergieren, wurde am Beispiel des Konsums aufgezeigt, dass nudging eine starke Tendenz dazu hat, privatheitliche Standards informationeller Natur zu ignorieren bzw. zu unterminieren. Insofern kann die der Arbeit zugrundeliegende Fragestellung folgendermaßen beantwortet werden: Nudging arbeitet informationellen Privatheitsrechten entgegen, weil es aus seiner Logik heraus, den nur begrenzt rational agierenden Konsument_innen die für sie beste Konsumentscheidung zu präsentieren, ein möglichst genaues Bild von ihnen benötigt. Dies hat zur Folge, dass unter dem Mantel der Nutzenmaximierung Fragen nach informationeller Privatheit nur eine untergeordnete Relevanz zugesprochen wird, während die Praxis des Datensammelns ein umso höheres Maß an Legitimität genießt.

Offen bleibt die Frage, wie dazu beigetragen werden könnte, informationeller Privatheit im Kontext des Konsums mehr
Geltung zu verschaffen. Ignoriert wurde in diesem Zusammenhang die seit Mai 2018 rechtsgültige Datenschutz-Grundverordnung, die mit dem Anspruch auftritt, datenschutzrechtliche Fragen mit mehr Sensibilität zu behandeln. Zumindest kann an dieser Stelle erwähnt werden, dass sie beispielsweise dem Anspruch der Rechtswissenschaftler_innen Roßnagel et al. (2018) nicht gerecht wird.

Auch bleibt die Frage offen, ob es sinnvoll ist, Privatheit als Wert hochzuhalten bzw. ihn überhaupt als relevant zu erachten. Gerade Vertreter_innen von Post-PrivacyAnsätzen sehen sie in Zeiten der Digitalisierung unwiederbringlich verloren. Sie plädieren deshalb für einen offensiven Umgang mit der daraus entstandenen Offenheit, der - so ihre These - durchaus sehr gewinnbringend und produktiv sein kann (wohl am einschlägigsten dazu Heller 2011; für eine wissenschaftliche Position pro Post-Privacy Hagendorff 2017). Ob das der richtige Umgang mit Privatheit ist, muss an dieser Stelle offengelassen werden. 


\section{ZUM AUTOR}

Enrico Hoerster studiert an der Universität Kassel Soziologie im Master. Besonders interessiert er sich für Poetologien des Sozialen, Sozial- und Gesellschaftstheorien sowie qualitative Methoden.

Der Beitrag wurde von Claas Pollmanns, Wibke Liebhart und Felix Fink gereviewed. Wibke Liebhart betreute den Überarbeitungsprozess und Sam Schneider und Eva-Maria Bub haben den Artikel lektoriert.

\section{LITERATUR}

Beck, Hanno (2018): Nudging. In: wisu. das wirtschaftsstudium, Jg. 47/2, S. 220-225.

Bröckling, Ulrich (2017): Nudging: Gesteigerte Tauglichkeit, vertiefte Unterwerfung. In: Bröckling, Ulrich (Hrsg.): Gute Hirten führen sanft. Über Menschenregierungskünste. Berlin: Suhrkamp, S. 175-196.

Broder, John M. (2012): Powerful Shaper of U.S. Rules Quits, With Critics in Wake. In: „The New York Times“, 03.08.2012, Online verfügbar unter New York Times, https://www.nytimes.com/2012/08/04/science/earth/cass-sunstein-to-leave-top-regulatory-post.html (19.04.2018).

Bundesregierung.de (2017): Mit Bürgern für Bürger. Online verfügbar unter, https://www.bundesregierung. de/Content/DE/StatischeSeiten/Breg/wirksam-regieren/2017-06-21-mit-buergern-fuer-buerger-regierungsstrategie-wirksam-regieren.html;jsessionid=9924F41C72367FAE8F932A9B4E86408A.s5t1?nn=2158204 (16.05.2018).

Ekardt, Felix (2017): Nudging. Angestupst in die Katastrophe. In: „Die Zeit“, 25.12.2017, Online verfügbar unter Zeit Online, https://www.zeit.de/wirtschaft/2017-12/ nudging-umweltschutz-richard-thaler-konsumenten/ komplettansicht (23.04.2018).

Foucault, Michel (1992): Was ist Kritik? Berlin: Merve.

Foucault, Michel (2004a): Geschichte der Gouvernementalität II. Die Geburt der Biopolitik. Vorlesung am Collège de France 1978-1979. Frankfurt am Main: Suhrkamp.

Foucault, Michel (2004b): Geschichte der Gouvernementalität I. Sicherheit, Territorium, Bevölkerung. Vorlesung am Collège de France 1977-1978. Frankfurt am Main: Suhrkamp.

Goodwin, Tom (2012): Why We Should Reject, Nudge‘. In: Politics, Jg. 32/2, S. 85-92.

Hagendorff, Thilo (2017): Das Ende der Informationskontrolle. Zur Nutzung digitaler Medien jenseits von Privatheit und Datenschutz. Bielefeld: transcript.

Hansen, Pelle Guldborg/Jespersen, Andreas Maaløe (2013): Nudge and the Manipulation of Choice. A Framework for the Responsible Use of the Nudge Approach to 
Behaviour Change in Public Policy. In: European Journal of Risk Regulation, Jg. 4/1, S. 3-28.

Heller, Christian (2011): Post-Privacy. Prima leben ohne Privatsphäre. München: C.H. Beck.

Högy, Kevin (2014): Libertärer Paternalismus und Nachhaltiger Konsum. Können Unternehmen als Entscheidungsarchitekten nachhaltigen Konsum fördern? Unveröffentlichte Bachelorthesis. Online verfügbar unter, https:// gdff.de/wp-content/uploads/2015/04/BA_KevinHoegy. pdf (28.06.2018).

Kapsner, Andreas/Sandfuchs, Barbara (2015): Nudging as a Threat to Privacy. In: Review of Philosophy and Psychology, Jg. 6/3, S. 455-468.

Law \& Policy (2016): Special Issue on Nudge, Jg. 38/3.

Leigh Star, Susan (2017): Dies ist kein Grenzobjekt: Reflexionen über den Ursprung eines Konzepts (2010). In: Gießmann, Sebastian/Taha, Nadine (Hrsg.): Susan Leigh Star. Grenzobjekte und Medienforschung. Bielefeld: transcript, S. 213-228.

Leigh Star, Susan/Griesemer, James R. (2017): Institutionelle Ökologie, ,Übersetzungen` und Grenzobjekte: Amateure und Professionelle im Museum of Vertebrate Zoology in Berkeley, 1907-39 (1989). In: Gießmann, Sebastian /Taha, Nadine (Hrsg.): Susan Leigh Star. Grenzobjekte und Medienforschung. Bielefeld: transcript, S. 81-115.

Piasecki, Stefan (2017): Schubs mich nicht! Nudging als politisches Gestaltungsmittel. In: „Bundeszentrale für politische Bildung“. Online verfügbar unter, https:// www.bpb.de/lernen/digitale-bildung/werkstatt/258946/ schubs-mich-nicht-nudging-als-politisches-gestaltungsmittel?type $=$ galerie $\&$ show $=$ image $\& \mathrm{i}=258949(23.04 .2018)$

Review of Philosophy and Psychology (2015): Nudge, Jg 6/3.

Roßnagel, Alexander u.a. (2018): Nationale Implementierung der Datenschutz-Grundverordnung. Herausforderungen - Ansätze - Strategien. Online verfügbar unter, https://www.forum-privatheit.de/forum-privatheit-de/ publikationen-und-downloads/veroeffentlichungen-des-forums/positionspapiere-policy-paper/Policy-Paper-Nationale-Implementierung-der-DSGVO_DE.pdf (22.05.2018).

Rössler, Beate (2001): Der Wert des Privaten. Frankfurt am Main: Suhrkamp.
Sunstein, Cass R. (2015): The Ethics of Nudging. In: Yale Journal on Regulation, Jg. 32/2, S. 413-450.

Thaler, Richard H./Sunstein, Cass R. (2003): „Libertarian Paternalism“. In: The American Economic Review, Jg. 93/2, S. 175-179.

Thaler, Richard H./Sunstein, Cass R. (2009): Nudge. Improving decisions about health, wealth and happiness. London: Penguin.

Thorun, Christian u.a. (2016): Nudge-Ansätze beim nachhaltigen Konsum. Ermittlung und Entwicklung von Maßnahmen zum „Anstoßen“ nach haltiger Konsummuster. Berlin: Umweltbundesamt.

Wilkinson, Martin (2013): Nudging and Manipulation. In: Political Studies, Jg. 61/2, S. 341-355. 


\section{Eine Opposition \\ gegen Autoritäts- \\ systeme des Marktes?}

\section{Wie kleine Produzent_innen Marktanteile erobern und so den Konsument_innen helfen}

Der Soziologe Ulrich Beck hatte einmal die Konsument_innen als „schlafende Riesen“ (Beck 2009: 131) bezeichnet. In diesem Sinne schrieb die Journalistin Tanja Busse (2006, 2007), Konsument_innen könnten die Produktion bestimmen, wenn sie nur die Augen öffnen. Da die Konsument_innen aber schliefen, seien sie für Missstände der globalisierten Wirtschaft verantwortlich, nämlich für die Missachtung von Umwelt- und Sozialstandards - also etwa für Hühner in Käfigen oder für versklavte Näherinnen. Dieser Beitrag zeigt an einem Beispiel, dass Konsument_innen nicht - wie behauptet - auf dem Markt souverän sein können, dass sie also nicht einfach so in der Lage sind, die Produktion zu lenken und ihre gewünschten Produkte zu bekommen. Souverän sind eher die Produzent_innen als die Konsument_innen. Letztere schlafen nicht, wie der Vorwurf gegen sie lautet: Sie werden von den Produzent_innen einfach nicht ausreichend über die Produkte informiert. Im Beispiel geht es um die Schwierigkeiten bei der Einführung neuer Lebensmittel in den USA, nämlich von Fleisch und Milch von Weideland- oder Freilandrindern: Am Anfang gab es keine Nachfrage von Konsument_innen, auf die die Produzent_innen reagierten - es gab nur ein Angebot von kleinen Produzent_innen, die sich bei den Konsument_innen erst mühsam einen Markt schaffen mussten. Mit ihrer Graswurzel-Initiative, einer Bewegung von unten, halfen diese Produzent_innen vielen Konsument_innen dabei, ein alternatives Angebot an Fleisch und Milch zu bekommen.

\section{Schlagwörter}

Konsument_innensouveränität; Nachhaltigkeit; Produzent_innensouveränität;

Verantwortung der Konsument_innen 
Tanja Busse (2006; 2007): Nur Konsument_innen können die Welt verbessern

Eigentlich, so die Journalistin Tanja Busse (2006: 22) in ihrem Buch Die Einkaufsrevolution. Konsumenten entdecken ihre Macht, sei alles ganz einfach: Konsument_innen müssten „nur die Augen öffnen“, um zu merken, dass ihre Einkäufe „auch jetzt bestimmen, welche Waren gerade auf welche Art und Weise produziert werden“. Nur weil die Verbraucher_innen Eier von Hühnern aus Käfigen kaufen, gebe es Hühner in Käfigen; nur weil die Konsument_innen beim T-Shirt-Kauf nicht nach Sozialstandards fragen, würden Näherinnen „wie Sklavinnen“ (ebd.) gehalten. Die Käufer_innen müssten endlich, sagt Busse (2007: 32), „die Verantwortung für ihren Konsum anerkennen“.

Nach Busse (2006: 22) gleichen die Verbraucher_innen „einem Stier, der sich von einem Lattenzaun bremsen lässt, weil er nicht weiß, wie stark er ist“. Und Busse (2006: 21) erinnert an ein ähnliches Bild des Soziologen Ulrich Beck, nämlich an das vom "schlafenden Riesen“ (Beck 2009: 131). Beck hatte in seinem 2002 erschienenen Buch Macht und Gegenmacht im globalen Zeitalter geschrieben: „Der schlafende Riese Konsument erwacht und verwandelt den Kaufakt in eine Abstimmung über die weltpolitische Rolle der Konzerne [...].“ (2009: 131) Dadurch, dass die Verbraucher_innen bestimmte Produkte nicht mehr kaufen, würden die Konzerne getroffen.

Nur die Verbraucher_innen - und sonst niemand - könnten durch ihre Kaufentscheidungen soziale Standards bei den Arbeitsbedingungen in den Herstellungsländern durchsetzen, behauptet Busse (2007: 32) in ihrem Aufsatz Die Entdeckung der Konsumentensouveränität: „Solange es keine verbindlich festgelegten sozialen Mindeststandards gibt, wird das so bleiben - es sei denn, die Konsumenten setzen diese Standards." Die Konsument_innen müssten, so Busse (2006: 21), einfach zu vielen Produkten sagen: „Verändere dich, sonst kauf ich dich nicht!" So könnten die Verbraucher_innen „die globalisierte Wirtschaft [...] verändern“, und zwar nach ihren Maßstäben. (ebd.) Busse (2006: 241) freut sich:

Und das Schöne ist: Die Weltverbesserung durch Konsum ist einfach zu haben: Es ist völlig ungefährlich, ein bestimmtes Produkt zu kaufen oder zu boykottieren - anders als ein Streik erfordern boycott und buycott kein persönliches Risiko. Oft ist es nicht einmal teurer, die korrekten Waren zu kaufen.

Nach Busse können die Verbraucher_innen also mit etwas gutem Willen korrekte Produkte kaufen - aber wollen sie das überhaupt? Busse (2007: 35) verweist auf eine 
Studie des Imug-Instituts 2003, in der 1.000 Menschen in Deutschland befragt wurden. Etwas mehr als 50 Prozent der Befragten sagten, sie würden - bei gleichem Preis und gleicher Qualität - lieber Produkte von Unternehmen kaufen, die „verantwortungsvoll agieren" (ebd.). Dieses Ergebnis ist für Busse nicht unbedingt „viel versprechend“ (ebd.); bedeute es doch, dass etwa „die Hälfte der Deutschen“"verantwortungsvolle Unternehmen „nicht bevorzugen“ würden, „auch wenn es sie weder Geld noch Qualität kosten würde“ (ebd.). Busse (2007: 36) erklärt das Ergebnis so: In Deutschland sei es der Wille der Bürger_innen, „dass sich der Staat darum kümmert", dass die Verbraucher_innen "sorglos einkaufen“ können. Die Verbraucher_innen sehen es also als staatliche Aufgabe an - und nicht als ihre Aufgabe -, auf die Standards der Produkte zu achten. Der Staat - so die Meinung der Verbraucher_innen - müsse sicherstellen, dass nur Produkte angeboten werden, die gewisse Standards erfüllen; so könnten die Kund_innen bedenkenlos bei jedem Produkt zugreifen. In der Realität könnten die Verbraucher_innen laut Busse (ebd.) aber nicht jedes Produkt sorglos kaufen. So habe Edda Müller (2005: 101), die damalige Vorsitzende des Bundesverbands der Verbraucherzentralen, kritisiert, dass den Verbraucher_innen „Informationen über Herstellungsbedingungen von Produkten vorenthalten" werden. Zum Beispiel, so Müller (ebd.), werde nicht darüber informiert, ob Lebensmittel von
Tieren stammen, „die mit gentechnisch veränderten Futtermitteln gefüttert worden sind“. Busse (2007: 36) fordert daher „mehr Transparenz“, um „ethisch korrektes Einkaufen" zu ermöglichen: Per Gesetz müsse vorgeschrieben werden, die Ergebnisse von Lebensmittelkontrollen zu veröffentlichen. Auch müsse es „verbindliche und genaue Angaben zu den Herstellungsbedingungen der Waren" (ebd.) geben. Als Beispiel dafür, wie „mit wenig Aufwand“ (ebd.) viel erreicht werden könne, nennt Busse die Kennzeichnung bei Eiern: Alle Verbraucher_innen könnten die Unterschiede verstehen, leicht (im gleichen Regal) zwischen den Kategorien wählen und müssten für die „fairen“ Eier nicht wesentlich mehr bezahlen.

\section{Fragen zu den Thesen von Tanja Busse und von Ulrich Beck}

Die Thesen von Tanja Busse - und die von Ulrich Beck - werfen viele Fragen auf: Stimmt es, dass die Verbraucher_innen schlafen? Kaufen sie die falschen Produkte nur deswegen, weil ihnen Informationen vorenthalten werden? Würden sie bei entsprechender Information richtig einkaufen? Haben die Verbraucher_innen überhaupt eine Verantwortung für ihren Konsum, die sie anerkennen müssen? Und sind Konsument_innen, die richtig kaufen, wirklich Stiere oder Riesen, die bestimmen können, „welche Waren gerade auf welche Art und 


\section{Stimmt es, dass die Verbraucher_innen schlafen? Kaufen sie die falschen Produkte nur deswegen, weil ihnen Informationen vorenthalten werden? Würden sie bei entsprechender Information richtig einkaufen?}

Weise produziert werden" (Busse 2006: 22)? Können sie - und nur sie - soziale Standards durchsetzen und sogar die Weltwirtschaft verändern? Sind die Konsument_innen in diesem Sinne souverän? Oder bestimmen im Gegenteil nicht eher die Produzent_innen, was wie produziert wird? Enthalten die Produzent_innen ihren Kund_innen nur Informationen vor - oder blockieren sie noch dazu gute Produkte, die die Kund_innen gerne hätten? Welche Verantwortung haben eigentlich die Produzent_innen - und wie können sie dieser Verantwortung gerecht werden? Soll sich der Staat nur darauf beschränken, Gesetze zur Information der Verbraucher_innen zu erlassen? Oder können die Verbraucher_innen doch erwarten, dass sich der Staat auch um die Standards kümmert?

\section{Armin Grunwald (2002; 2010): Macht der Konsument_innen liegt nicht in ihrem Konsum, sondern in ihrem politischen Engagement}

Der Physiker und Philosoph Armin Grunwald widerspricht einigen der von Beck und Busse vertretenen Thesen, und zwar in seinen Aufsätzen Die Realisierung eines Nachhaltigen Konsums - Aufgabe der Konsumenten? (2002) und Wider die Privatisierung der Nachhaltigkeit (2010). Nach Grunwald (2002: 433f.) bedeutet „Nachhaltigkeit“, dass künftige Generationen gleiche Lebenschancen und Entfaltungsmöglichkeiten vorfinden sollen wie die heutige Generation; die wirtschaftlichen und sozialen Lebensbedingungen müssten mit Rücksicht auf die natürlichen Lebensgrundlagen verbessert werden, damit diese Lebensgrundlagen auch langfristig gesichert werden. Wie schon die Überschriften seiner Aufsätze sagen, wendet sich Grunwald dagegen, Nachhaltigkeit zur Privatsache der Konsument_innen zu erklären.

In seinem Aufsatz Wider die Privatisierung der Nachhaltigkeit schreibt Grunwald (2010: 182): Die Macht der Konsument_ innen ,liegt nicht in ihrer Konsummacht [...], sondern in ihrer politischen Macht“. Beck (2009: 131) hatte dagegen gesagt, dass die Konsument_innen die Konzerne durch „Nicht-Kauf“" treffen könnten; und Busse hatte behauptet, dass die Verbrau- 
cher_innen die "globalisierte Wirtschaft [...] verändern" (Busse 2006: 21) und eine „Weltverbesserung durch Konsum“ (Busse 2006: 241) erreichen könnten. Weiter schreibt Grunwald (2010: 182), dass Becks Rede vom „schlafenden Riesen“ nur insofern stimme, als dass Konsument_innen „wirklich schlafen“. Damit meint Grunwald, dass Menschen - als Konsument_innen - keine Riesen sind: Grunwalds These ist, dass die Menschen mit ihrem Konsum die Wirtschaft gar nicht lenken können - ihre Nachfrage bestimme nicht, wie Busse es formulierte, die Art und Weise der Produktion.

Zwar werde - „wenn Menschen nach Maßstäben der Nachhaltigkeit konsumieren" - erwartet, dass „das Wirtschaftssystem aus Eigeninteresse zunehmend nachhaltige Produkte anbieten“ und „sich schließlich ganz auf diese Art des Konsums einstellen “ werde (Grunwald 2010: 179). In dieser Erwartung stecken aber, so Grunwald (ebd.), „eine Reihe von problematischen Prämissen“. Grunwald geht somit nicht davon aus, dass eine Nachfrage nach nachhaltigen Gütern automatisch ein solches Angebot schafft. Trotz einer solchen Nachfrage könne das entsprechende Angebot fehlen. Auch wenn also die Konsument_innen nachhaltig konsumieren wollten, sei nicht garantiert, dass die Produzent_innen ein entsprechendes Angebot an nachhaltigen Gütern bereitstellen. In diesem Sinne betont Grunwald (2002: 443): „Ohne ein ent- sprechendes Angebot hat der Konsument keine Möglichkeit, sich für nachhaltigen Konsum zu entscheiden, außer vielleicht durch Konsumverzicht." Von daher argumentiert Grunwald, dass die Macht der Konsument_innen nicht im Konsum liege - denn der Konsum bestimme nicht unbedingt das Angebot, lenke also nicht die Wirtschaft und laufe daher ins Leere.

Die Menschen seien aber „nicht nur Konsument(innen)“, so Grunwald (2010: 181) weiter, „sondern auch politische Akteure als Bürger(innen) ihrer Gemeinwesen und als Mitglieder der Zivilgesellschaft". Als politische Akteur_innen müssten die Menschen darauf hinwirken, dass das Wirtschaftssystem ,in eine ökologische Richtung ,gedrängt' wird“ (ebd.). Erst wenn sich die Verbraucher_innen in den politischen Debatten für die Umwelt engagierten, könnten ,die großen Nachhaltigkeitsprobleme ernsthaft in Angriff genommen werden“ (Grunwald 2010: 182). Zum Beispiel sollten Konsument_innen, die ökologisch schädliche Fernreisen unternehmen, keine Zertifikate kaufen, die die Aufforstung von Wäldern finanzieren. Vielmehr sollten sich diese Verbraucher_innen für eine Kerosinsteuer einsetzen: „Das würde die Rahmenbedingungen ändern, unter denen Flugreisen stattfinden", und zwar „allgemeinverbindlich“ (Grunwald 2010, 181). 


\section{Tibor Scitovsky (1976): Konsu- ment_innen bekommen nicht das, was sie wollen}

Grunwalds These lautet, dass aus der Nachfrage nach nachhaltigen Produkten nicht unbedingt ein entsprechendes Angebot resultiert; dass also die Produzent_innen nicht immer auf die Wünsche der Konsument_innen nach nachhaltigen Waren eingehen. Diese These - auch und gerade mit Blick auf nachhaltige Produkte - hat schon der Wirtschaftswissenschaftler Tibor Scitovsky sein Buch The Joyless Economy, das ein Jahr später auch in Deutschland erschien - unter dem Titel Psychologie des Wohlstands. In diesem Buch beschäftigt sich Scitovsky mit dem Markt der Konsumgesellschaft. Auf diesem Markt kaufen die Konsument_innen das, was die Produzent_innen verkaufen; die Handlungen von Käufer_innen und Verkäufer_innen sind daher „in Einklang“, wie Scitovsky (1977: 13) sagt. Diese Harmonie zwischen Käufer_ innen und Verkäufer_innen werde von bestimmten Wirtschaftswissenschaftler innen als Beweis dafür gesehen, „dass die von der Wirtschaft hergestellten Güter dem Geschmack der Konsumenten genau entsprechen“" (Scitovsky 1977: 13f.). Allerdings müsse sich eine Gruppe an die andere anpassen: Und zwar müssten sich, so Scitovsky, entweder die Produzent_innen an die Wünsche der Konsument_innen anpassen - oder aber die Konsument_innen

an das Angebot der Produzent_innen. „Grundsätzlich“, meint Scitovsky (1977: 14), hätten die Produzent_innen „mehr Macht und Einfluss" als die Konsument innen. Die Übereinstimmung zwischen Konsument_innen und Produzent_innen ihre Harmonie - könne daher auch einfach ein "Zeichen“ sein „für die Anpassung“ der Konsument_innen an die „Anforderungen des Produktionssystems" (ebd.). In diesem Fall könnten „wir uns kaum dazu beglückwünschen“ (ebd).

\section{Scitovskys Beispiel für die Hilf- losigkeit der Konsument_innen gegenüber der Entscheidungs- gewalt der Produzent_innen: US-Kleinwagen ab den 1960er Jahren}

Scitovsky (1977: 232) beklagt die Hilflosigkeit der Konsument_innen gegenüber den Produzent_innen. Das Dilemma bestehe darin, dass die Produzent_innen andere Interessen als die Konsument_innen haben und „dass die Auffassung des Produzenten über das, was für den Konsumenten richtig ist, von seinen Vorstellungen über das, was ihn als Produzent befriedigt, beeinflusst wird“" (Scitovsky 1977: 231). Einfacher gesagt: Die Produzent_innen halten ein Produkt schon dann für optimal für die Konsument_innen, wenn es für sie selbst optimal ist. 
Für die erwähnte Hilflosigkeit der Konsument_innen gegenüber der Entscheidungsgewalt der Produzent_innen gibt Scitovsky (1977: 232) folgendes Beispiel: Nach dem Zweiten Weltkrieg seien die Autos in den USA ,länger, schwerer und protziger“ geworden - und die US-Autoindustrie sagte, dass dies die "genau erforschten Wünsche" der Amerikaner widerspiegele. Die Industrie habe behauptet, „dass die Öffentlichkeit die kürzeren und leichteren Autos von gestern nicht mehr haben wollte" (ebd.). Erst dadurch, dass die US-Amerikaner_innen ausländische Autos bestellten, die durchschnittlich 1,20 Meter kürzer waren als die einheimischen Modelle, konnten sie ihre Vorliebe für kleinere Autos zeigen: Die Importe stiegen von 0,5 Prozent Marktanteil im Jahr 1948 auf 22 Prozent Marktanteil im Jahr 1974. Erst in den 1960er Jahren reagierte die US-Autoindustrie, indem sie zunächst Kompaktwagen und dann Kleinwagen auf den Markt brachte. Diese beiden Wagentypen erreichten 1970 in den USA bereits einen Marktanteil von 40 Prozent. Scitovsky will mit seinem Beispiel zeigen, dass die Autoindustrie lange nicht auf die Nachfrage der US-Konsument_ innen reagiert habe; bis in die 1960er Jahre mussten Autofahrer_innen, die Kleinwagen haben wollten, sich diese aus dem Ausland bestellen. Wie viele Kund_innen sich ein kleineres Auto gewünscht hatten, sei deutlich geworden, als im Jahr 197040 Prozent der Kund_innen die US-Kleinund Kompaktwagen wählten, nachdem

\section{9 Die Produzent_ innen halten ein Produkt schon dann für optimal für die Konsument_innen, wenn es für sie selbst optimal ist.}

diese endlich von der amerikanischen Autoindustrie angeboten wurden.

\section{Vance Packard (1957): Viele Amerikaner_innen wollten kleine Autos, bekamen sie aber nicht}

Auch der amerikanische Journalist Vance Packard (1966: 156) hat in seinem 1957 erschienenen Buch The Hidden Persuaders (dt. Die geheimen Verführer) gezeigt, dass sich viele Amerikaner_innen schon „Anfang der fünfziger Jahre" kleinere Autos gewünscht hatten: Die Autoindustrie sei „mit Vorschlägen, einen kleinen, leistungsfähigen, billigen Wagen herauszubringen", überschüttet worden. Autofirmen hätten daraufhin in einer Studie Konsument_ innen nach ihren Wünschen befragt. Diese Studie sei zu dem Ergebnis gekommen, dass „nur eine Minderheit an Kleinwagen interessiert sei“ (ebd.). Packard bestätigt mit seinem Bericht Scitovskys Sicht: Viele 
Amerikaner_innen hatten sich - aus verschiedenen Gründen - einen US-Kleinwagen gewünscht; dieser Wunsch wurde aber von den Produzent_innen nicht berücksichtigt.

\section{Das zerstörte Selbstbild des souveränen Konsumenten}

Scitovsky (1977: 12) sagt, dass sich "das Bild des amerikanischen Verbrauchers in der kurzen Zeitspanne eines Jahrhunderts grundlegend gewandelt" habe: Früher hätten sich sowohl die Verbraucherin als auch der Verbraucher als souveräne Konsument_innen gesehen; mit der Zeit aber sei diese "stolze Selbstdarstellung" geschrumpft - und zwar „zu dem bemitleidenswerten Bild eines hilflosen Verbrauchers, der von allen Seiten schikaniert und belästigt, betrogen und übers $\mathrm{Ohr}$ gehauen und sogar vergiftet wird.“ (ebd.)

Zwar hätten die Käufer_innen in den USA das „Recht auf freie Wahl“ und die „Freiheit zu entscheiden, was und wieviel sie kaufen " (Scitovsky 1977: 15). Aber, so Scitovsky (ebd.): „Diese Freiheit darf man jedoch nicht mit der Konsumentensouveränität verwechseln."Die_der Konsument_in sei erst dann souverän, „wenn seine Wahl die Art und Menge der produzierten Güter und Dienstleistungen beeinflusst“ (ebd.). Scitovsky unterscheidet also zwischen der Freiheit der Konsument_innen, zwischen verschiedenen Produkten zu wählen, und der Souveränität der Konsument_innen, das Angebot tatsächlich beeinflussen zu können. Mit „Konsument_innensouveränität" meint Scitovsky also, dass den Konsument_innen fehlende Produkte wenn sie denn gewünscht werden - von den Produzent_innen zur Verfügung gestellt werden. Die Konsument_innenfreiheit hält Scitovsky für gegeben, nicht aber die Konsument_innensouveränität: Zwar, so Scitovsky (1977: 16), werde von anderen behauptet, dass es in den USA diese „volle Konsumentensouveränität" gebe, dass also die Wahl der Konsument_innen beim Einkaufen die Produktion bestimme. Diese Ansicht nennt Scitovsky (ebd.) aber nur eine „stolze Behauptung“ - eine Behauptung also, die nicht zutrifft.

Wie Grunwald ist Scitovsky nicht der Ansicht, dass die Verbraucher_innen mit ihren Wünschen ein entsprechendes Angebot erzeugen könnten: Die Verbraucher_ innen haben nur die Wahl, Sachen nicht zu kaufen - allein dadurch erscheinen aber alternative Produkte noch nicht auf dem Markt. In Scitovskys Beispiel würde das heißen, dass die US-Autofahrer_innen nur die Wahl hatten, kein großes US-Auto zu kaufen - also auf ein amerikanisches Auto zu verzichten und stattdessen ein ausländisches Auto zu kaufen. Diese Ablehnung der großen US-Autos bei vielen Konsument_innen führte aber nicht direkt dazu, dass die US-Autoindustrie kleinere 
Autos anbot; die Ablehnung erzeugte also kein entsprechendes Angebot. Daher sagt Scitovsky, dass die Verbraucher_innen nicht souverän sind: Sie können zwar zwischen verschiedenen Angeboten wählen - sofern der Markt überhaupt Alternativen bietet -, aber nicht die Produktion bestimmen. Busse (2006: 22) hatte dagegen behauptet, dass die Konsument_innen „bestimmen, welche Waren auf welche Art und Weise produziert werden“. Für Busse sind die Konsument_innen souverän, für Scitovsky nicht.

Wer hat nun Recht, Busse oder Scitovsky und Grunwald? Können die Konsument_ innen für die Produktion neuer Waren sorgen, sind sie daher souverän - oder nicht? Diese Frage soll anhand von einem Beispiel verdeutlicht werden. Und zwar geht es in diesem Beispiel um Fleisch und Milchprodukte von Weidelandrindern in den USA.

\section{Klaus Weber, Kathryn L. Heinze und Michaela DeSoucey (2008): Farmer_innen und Autor_innen haben zusammen einen neuen Markt geschaffen}

2006 und 2007 führte der deutsch-amerikanische Wirtschaftswissenschaftler Klaus Weber zusammen mit Kathryn L. Heinze und Michaela DeSoucey 41 Interviews mit Farmer_innen, Journalist_innen und Verbraucher_innen zum Thema Weidelandrinder. In den 1990er Jahren, so Weber, Heinze und DeSoucey (2008: 533), hielten nur „wenige Dutzend“ Farmer_innen in den USA Rinder auf der Weide, wo sie mit Gras gefüttert wurden (grass-fed). Fast alle Rinder in den USA lebten dagegen in engen Gehegen (feedlots), wo sie mit Mais gemästet wurden (corn-fed). 2006 gab es schon etwa 2000 grass-fedFarmer_innen, die einen US-Marktanteil von $0,2 \%$ erreichten. In den 1990er Jahren waren gemästete Tiere teurer als Tiere von der Weide gewesen. Es hieß damals, das Fleisch der Masttiere sei von besserer Qualität: Es sei zarter und saftiger, die Milch dieser Tiere sei fetter; dagegen wurde das grass-fed-Fleisch für zäh und 
geschmacklos gehalten (Weber et al. 2008: 553, 555). Diese Sicht kehrte sich etwa ab dem Jahr 2000 um: Auf einmal war Weidevieh teurer als Mastvieh. Weber, Heinze und DeSoucey (2008: 533) kommen zu dem Schluss, dass eine Graswurzel-Bewegung verschiedener Akteur_innen (,grassroots coalition movement") diesen neuen grass-fed-Markt geschaffen habe; dabei sei es den Akteur_innen um nachhaltige Landwirtschaft, ländliche Entwicklung und Gesundheit gegangen. Diese Bewegung sei eine Opposition gewesen zu einem „System industrieller Landwirtschaft“ (ebd.), das seit dem Zweiten Weltkrieg „dominant" gewesen sei. Frühe Aktivist_innen waren Allan Nation, der eine Zeitschrift für grass-fed-Farmer herausgab, und der Farmer Joel Salatin, der mehrere Bücher zum Thema veröffentlichte. In den späten 1990er setzten sich auch die Journalistin Jo Robinson und der Biologe Allan Savory für die grass-fed-Initiative ein. Der New Yorker Gastro-Kritiker Marian Burros lobte das Weidelandfleisch, und Michael Pollan schrieb den Bestseller über Ernährung The Omnivore's Dilemma, in dem er auf das grass-fed-Fleisch hinwies.

\section{Die Nachhaltigkeit bei der grass-fed-Zucht aus Sicht eines Farmers}

Im Interview mit Weber und seinen Kolleginnen (2008: 548) sprach ein Farmer von „riesigen Vorteilen“ der grass-fed- gegenüber der corn-fed-Methode. Er sagte, das Problem bei der corn-fed-Methode sei, dass die Gehege überfüllt seien und die Tiere immer im Staub und im Schlamm lebten. Dadurch würden viele Tiere krank. Weidende Tiere dagegen blieben gesund, so dass auf Antibiotika und Hormone im Futter verzichtet werden könne.

\section{Hürden für die grass-fed- Farmer_innen: Blockierte Produktions- und Vertriebs- kanäle und desinteressierte Verbraucher_innen}

Neue Märkte stehen im Gegensatz zu institutionalisierten Produktionssystemen (,institutionalized production regimes“), so Weber, Heinze und DeSoucey (2008: 543). Daher habe es auch für die grassfed-Farmer_innen "Herausforderungen“ gegeben, die für Unternehmer_innen in neuen Märkten typisch seien (ebd.). So mussten die grass-fed-Farmer_innen zunächst Restaurants davon überzeugen, dass sie ihr Fleisch kaufen. Auch sonst mussten die Farmer_innen „eine Reihe von Hürden“ überwinden, schreiben Weber, Heinze 
und DeSoucey (2008: 554): Die meisten Schlachthäuser waren daran gewöhnt, viele Tiere auf einmal zu schlachten; die Schlachtung weniger grass-fed-Tiere wurde von Schlachthäusern oft abgelehnt. Ein grass-fed-Farmer sagte im Interview, dass er gerne mit anderen zusammen ein eigenes Schlachthaus bauen würde. Auch die Auflagen der Behörden orientierten sich an großen Farmen; für kleinere Farmer_innen waren sie überproportional teuer. Viele Lebensmittelgeschäfte weigerten sich, grass-fed-Fleisch zu verkaufen, weil sie nur an großen Mengen Fleisch interessiert waren. Ein Farmer zeigte sich im Interview skeptisch gegenüber dem Verkauf in einer Lebensmittelkette: „Werden sie unser Produkt so vertreten und vermarkten, wie wir es machen würden? Wenn ihre Mitarbeiter irgendwie voreingenommen sind - wenn es Preisunterschiede gibt - wären sie dann in der Lage, den Unterschied zu erklären?“ (ebd.) Die meisten Farmer_innen mussten daher auf verschiedene alternative Verkaufskanäle zurückgreifen wie Hofläden, Märkte von Farmer_innen oder den Internetverkauf. Heute noch, so Weber und Kolleginnen (2008: 551), werde oft die Geschichte von der „schlechten Behandlung" der grass-fed-Farmer_innen durch die großen Lebensmittelketten berichtet.

Farmer_innen aus Colorado, Texas und Idaho klagten im Gespräch mit Weber, Heinze und DeSoucy (2008: 552) darüber, dass sich nicht genügend Konsument_ innen vor Ort für ihre Produkte interessierten. Weber und seine Kolleginnen stellten denn auch fest, dass sowohl das Interesse der US-Konsument_innen als auch ihr Wissen hinsichtlich der verschiedenen Zuchtmethoden „begrenzt“ (ebd.) war. Ein Farmer berichtete, dass er 1998 in einer großen Stadt keine ausreichende Nachfrage nach grass-fed-Produkten gefunden habe; bei seinem zweiten Versuch 2005 habe er dagegen Erfolg gehabt (Weber et al. 2008: 553). Diesen Umschwung führte der Farmer auf die geänderten Interessen und auf das geänderte Bewusstsein der Käufer_innen zurück.

\section{Sebastian Nessel (2016): Soziale Bewegungen wollen gegen Autoritätssysteme des Marktes vorgehen}

Webers, Heinzes und DeSouceys Studie zeige „den Einfluss sozialer Bewegungen für die Konstitution neuer Märkte“, schreibt der Soziologe Sebastian Nessel (2016: 100) in seinem Buch Verbraucherorganisationen und Märkte. Die Studie verdeutliche die Argumentation der grass-fed-Bewegung, „dass die Vermarktung von Fleischprodukten jenseits der industriellen Produktion lange Zeit durch das bestehende Autoritätssystem dieses Marktes blockiert wurde und Konsumenten demnach keine Nachfrage für neue Produkte entwickeln konnten “ (ebd.). Nessel (2016: 97) sieht denn auch 
ein Merkmal solcher sozialen Bewegungen darin, dass sie das Ziel haben, „dominante soziale und kulturelle Praktiken und Autoritätssysteme zu verändern“. Dass soziale Bewegungen - wie die grass-fed-Bewegung - sich behindert fühlen, und zwar von angeblich marktbeherrschenden „Autoritätssystemen“, ist also kein Einzelfall.

\section{Eigene Stellungnahme zum grass-fed-Beispiel: Konsument_ innen sind nicht ",souverän“}

Stimmt es, dass die etablierten Produzent_innen die grass-fed-Bewegung mit Absicht „blockiert" haben? Waren zum Beispiel die Einwände von Schlachthäusern und Lebensmittelhändlern, dass sie nur große Stückzahlen an Vieh akzeptieren könnten, nur vorgeschoben? Die Studie von Weber und seinen Kolleginnen gibt auf diese Frage keine Antwort. Auf jeden Fall ist aber deutlich geworden, wie schwer es die grass-fed-Farmer_innen am Anfang hatten, ihr Fleisch zu vermarkten.

Beck hatte geschrieben, dass die Konsument_innen schlafen; Busse und Grunwald hatten Beck darin Recht gegeben. Haben die US-Konsument_innen geschlafen, als 2006 immer noch fast 100 Prozent von ihnen das corn-fed-Fleisch kauften? Natürlich ist bedauerlich, dass - wie Weber und seine Kolleginnen sagen - das Interesse der Konsument_innen daran, wie ihre
Nahrung produziert wird, „,begrenzt“ war. Aber auch den Konsument_innen, die sich später für das grass-fed-Fleisch entschieden haben, lässt sich nicht vorwerfen, dass sie zuvor geschlafen hatten: Die Informationen zu den Zuchtmethoden waren nicht einfach so verfügbar. Im Geschäft jedenfalls wurde nicht auf den Unterschied zwischen corn-fed-und grass-fed-Methode hingewiesen - grass-fed-Fleisch wurde ja gar nicht angeboten. Und sogar wenn es den Verbraucher_innen gelungen wäre, sich selbst Informationen zu beschaffen, dann hätte ihnen das - mangels Angebot - zumindest im Laden keinen alternativen Konsum ermöglicht.

Konsument_innen, die nicht über Produktionsbedingungen aufgeklärt werden und die mangels Alternativen auch nicht zwischen verschiedenen Produkten auswählen können, sind meiner Ansicht nach nicht souverän. Solche Verbraucher_ innen können nicht die Art und Weise der Produktion bestimmen: Wenn sie noch nicht einmal wissen, welche Mängel das derzeitige Angebot hat - wie können sie dann wissen, wie ein besseres Angebot aussehen soll? Aber sogar wenn sie wüssten, welche Verbesserungen sie wollen, so ist zweifelhaft, ob ihnen dann die Produzent_innen ein entsprechendes Angebot machen würden. 


\section{Wilhelm Henrichsmeyer, Oskar Gans und Ingo Evers (1988): Nicht die Konsument_innen, sondern die Produzent_innen sind souverän}

Die Volkswirte Wilhelm Henrichsmeyer, Oskar Gans und Ingo Evers (1988: 307) schreiben in ihrem Buch Einführung in die Volkswirtschaftslehre, dass Waren eigentlich „entsprechend den Wünschen der Konsumenten" produziert werden sollen: Dann, so meinen die Autoren, seien die Konsument_innen souverän. „In der Realität“ sei aber diese „Souveränität“ der Konsument_innen „nicht unerheblich“, also erheblich „eingeschränkt“ (ebd.). Die Produzent_innen könnten nämlich die Präferenzen der Konsument_innen zum Beispiel durch Werbung beeinflussen und dadurch eine Manipulation der Konsument_innen herbeiführen. Auch könnten die Produzent_innen „die Einführung technischer Fortschritte verhindern“, „meist aus Gründen der Auslastung bestehender Produktionsanlagen“ (ebd.). Die Produzent_innen wollen also, so Henrichsmeyer und Kollegen, ihre vorhandenen Produktionsanlagen ausgelastet sehen - anstatt diese Anlagen im Sinne des technischen Fortschritts umzustellen. Aus den genannten Gründen spielten die Konsument_innen, so Henrichsmeyer und seine Kollegen, „bei der Bestimmung der Produktion“ nur „eine eher passive Rolle“ (ebd.). Die Nachfrage der Konsument_ innen sei „in vielen Fällen eher als Reflex“ auf die von den Produzent_innen „bestimmten Bedürfnisbefriedigungsmöglichkeiten" zu sehen (Henrichsmeyer et al. 1988: 308); die Nachfrage sei also nur eine Folge des Angebots. Nicht die Konsument_innen, sondern die Produzent_innen seien daher „souverän“; Henrichsmeyer und Kollegen sprechen von „Produzentensouveränität" (ebd.).

\section{Nicht die Konsument_innen, sondern die grass-fed-Produzent innen bestimmten das Angebot}

Auch ohne die Frage zu klären, ob Konsument_innen durch Werbung für corn-fed-Produkte manipuliert wurden, ist festzuhalten, dass es für die Verbraucher_innen eigentlich nur eine bestimmte „Bedürfnisbefriedigungsmöglichkeit" gab: nämlich das corn-fed-Fleisch im Supermarkt zu kaufen. Ihr Konsum war damit nur ein Reflex auf das vorhandene Angebot. Das Angebot konnten die Konsument_ innen alleine nicht bestimmen: Erst die grass-fed-Bewegung, angeführt von Farmer_innen und Journalist_innen, sorgte dafür, dass die Verbraucher_innen ein alternatives Angebot bekamen. Im Beispiel bestimmten also nicht die Verbraucher innen, sondern neue Produzent_innen die Art und Weise der Produktion auf dem Fleischmarkt, nämlich die Produktion von grass-fed-Fleisch für einen Teil des Marktes. 


\section{Wer nur von den Verbraucher_innen verlangt, die Welt zu verbessern, der entlässt Produzent_ innen und Regierungen vorschnell aus deren vorrangiger Verantwortung, für eine ethisch korrekte Wirtschaft zu sorgen.}

\section{Antworten auf die von Busse aufgeworfenen Fragen}

Busse (2007: 36) hatte „mehr Transpa-

renz" gefordert, um „ethisch korrektes Einkaufen“"zu ermöglichen. Wenn aber die Konsument_innen - ohne entsprechende Information - gar nicht ethisch korrekt einkaufen können, wie sollen sie dann die „Verantwortung“ für ihren Konsum übernehmen, wie Busse (2007: 32) auch gefordert hat? Die Konsument_innen sind - auch bei entsprechender Aufklärung über die Produkte - keine Stiere oder Riesen: Wie das Beispiel gezeigt hat, können sie ein Angebot, das es nicht gibt, nicht einfach herbeizaubern: Sie sind auf alternative Produzent_innen - wie im Beispiel auf die grass-fed-Farmer_innen - angewiesen, die nicht in jeder Branche bereitstehen. Wie soll es nur die Aufgabe der schwachen Verbraucher_innen sein, Standards für die Produktion durchzusetzen? Das ist erst einmal Sache der Produzent_innen und - wenn diese versagen - Sache des Staates und der Staatengemeinschaften. Die Verbraucher_innen können nicht nur erwarten, dass Staaten Gesetze erlassen, die Informationspflichten der Produzent_innen vorsehen, sondern auch, dass diese Staaten Mindeststandards für Produkte festsetzen. Wer nur von den Verbraucher_innen verlangt, die Welt zu verbessern, der entlässt Produzent_innen und Regierungen vorschnell aus deren vorrangiger Verantwortung, für eine ethisch korrekte Wirtschaft zu sorgen. 
ZUR AUTORIN

Soonim Shin, geboren in Daegu / Südkorea, studierte Germanistik in Daegu. Später studierte sie Soziale Arbeit in Mainz und wurde Diplom-Sozialarbeiterin (FH). Ihr Studium der Erziehungswissenschaft, Soziologie und Philosophie an der Johannes Gutenberg-Universität Mainz beendete Soonim als Magistra Artium (M. A.). Das Thema ihrer Magisterarbeit war „Moralische Erziehung bei Émile Durkheim“. Bei der Frühjahrstagung der Sektion Wirtschaftssoziologie der DGS im Juni 2018 in Graz hielt Soonim den Vortrag „Der ,ökonomische Nationalist' Max Weber ein Vater des heutigen, ökonomischen $\mathrm{Na}$ tionalismus'?" Und bei der ad-hoc-Gruppe zur Vergleichenden Wirtschaftssoziologie beim 39. Kongress der DGS sprach Soonim zum Thema „Die ,Moralisierung der Märkte - Trend und Ordnungsfaktor in der (Welt-)Gesellschaft".

Der Beitrag wurde von Andreas Schulz, Laura Porak und Tamara Schwertel gereviewed. Tamara Schwertel hat den Überarbeitungsprozess redaktionell betreut und Sam Schneider, Andreas Schulz und Tanja Strukelj haben den Artikel lektoriert.

\section{LITERATUR}

Beck, Ulrich (2009): Macht und Gegenmacht im globalen Zeitalter. Neue weltpolitische Ökonomie. Frankfurt am Main: Suhrkamp.

Busse, Tanja (2006): Die Einkaufsrevolution. Konsumenten entdecken ihre Macht. 3. Aufl. München: Karl Blessing.

Busse, Tanja (2007): Die Entdeckung der Konsumentensouveränität. In: Orientierungen zur Wirtschafts- und Gesellschaftspolitik Nr. 111. Bonn: Ludwig-Erhard-Stiftung, S. 32-36.

Grunwald, Armin (2002): Die Realisierung eines Nachhaltigen Konsums - Aufgabe der Konsumenten? In: Gerhard Scherhorn/Christoph Weber (Hrsg.): Nachhaltiger Konsum. Auf dem Weg zur gesellschaftlichen Verankerung. München: ökom, S. 433-442.

Grunwald, Armin (2010): Wider die Privatisierung der Nachhaltigkeit. Warum ökologisch korrekter Konsum die Umwelt nicht retten kann. In: GAIA - Ökologische Perspektiven für Wissenschaft und Gesellschaft, Jg. 19, S. 178-182.

Henrichsmeyer, Wilhelm/Gans, Oskar/Evers, Ingo (1988): Einführung in die Volkswirtschaftslehre. Stuttgart: UTB.

Müller, Edda (2005): Verbraucherpolitik als Querschnittsaufgabe profilieren. Interview mit Edda Müller von Jörn Lamla und Ansgar Klein unter Mitarbeit von Anja Löwe am 17. Oktober 2005 in Berlin. In: Forschungsjournal Neue Soziale Bewegungen, Jg. 18/4, S. 98-105.

Nessel, Sebastian (2016): Verbraucherorganisationen und Märkte. Eine wirtschaftssoziologische Untersuchung. Wiesbaden: Springer VS.

Packard, Vance (1966 [1957]): Die geheimen Verführer. Der Griff nach dem Unbewussten in Jedermann. 9. Aufl. Düsseldorf: Econ.

Scitovsky, Tibor (1977): Psychologie des Wohlstands. Die Bedürfnisse des Menschen und der Bedarf des Verbrauchers. Frankfurt am Main: Campus.

Weber, Klaus/Heinze, Kathryn L./DeSoucey, Michaela (2008): Forage for Thought. Mobilizing Codes in the Movement for Grass-fed Meat and Dairy Products. Administrative Science Quarterly 2008, Jg. 53/3, S. 529-567. 

ERSPETIVE
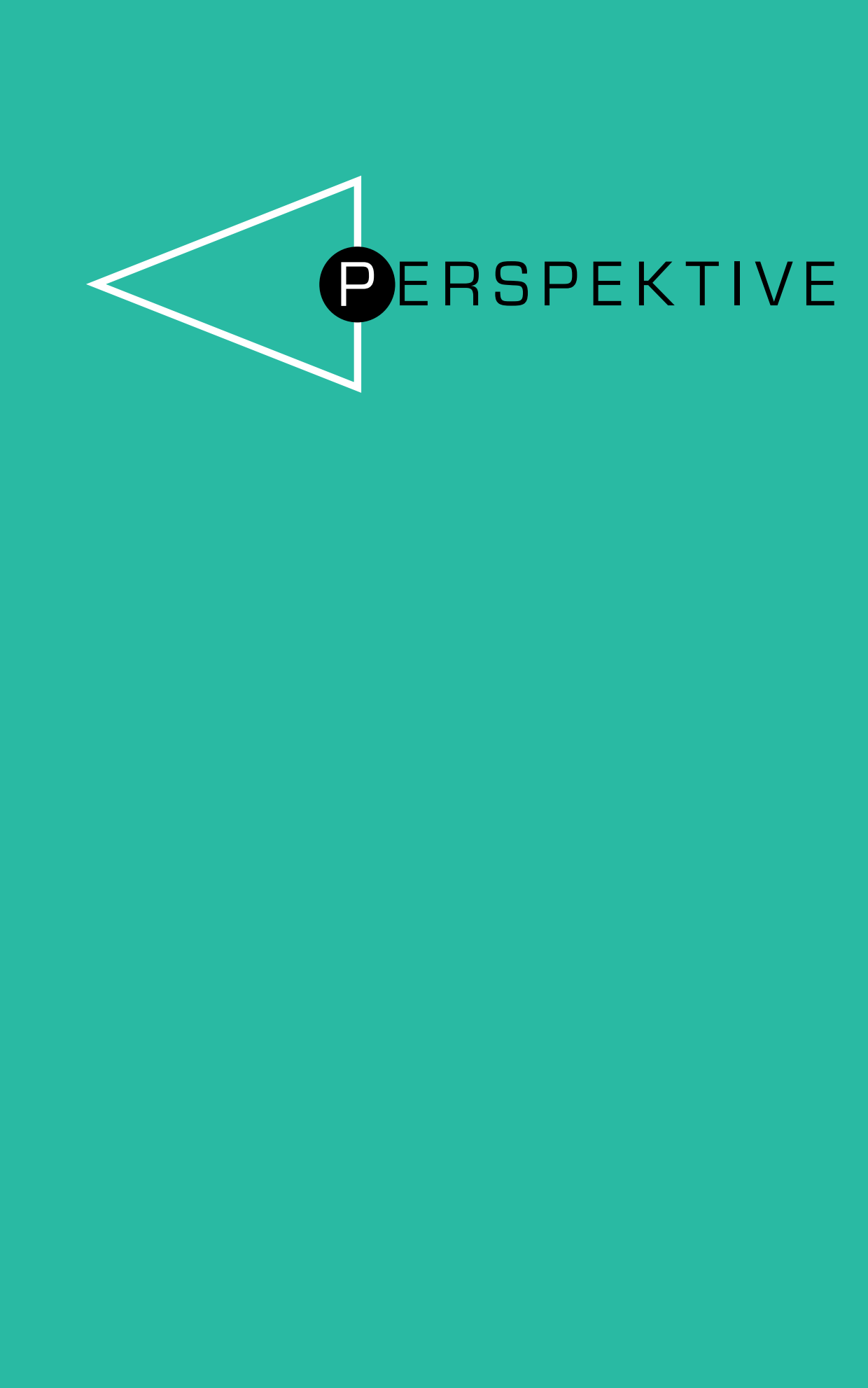

-

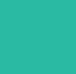




\section{Shopdropping}

\section{Materialities, Mobilities, Creative Interventions}

by Mara Mürlebach

This article brings together a feminist sloth, a cultural activist practice and a Human Geography student. That student, the author of this article, shopdropped a sloth sticker to protest the discriminatory body politics of diet magazines. Through a practice-based and autoethnographic approach, she tries to make sense of her own experience and of shopdropping as an activist practice. Drawing on the geographical concepts of place and movement, shopdropping is situated within the debates around subversive material cultures and creative interventions into places of consumption. Shopdroppers disobedient things lend themselves to thinking through the connections between geography, art, and activism and for contemplating the effects of creative engagements with cultures of consumption.

\section{Keywords}

shopdropping; material geographies; subversive material culture; disobedient things; consumption cultures 


\section{Introduction: Shop til you drop?}

How to shopdrop in ten steps... Yes, that sounds helpful, I think. I've been sitting in front of the computer for quite a while, searching the internet for references on shopdropping. I wildly click through the websites until... Wait a minute, this is funny! Two people shopdropped themselves, wearing huge costumes made of work gloves. They pretend to be a pile of gloves in a hardware shop - oh my God, THIS IS HILARIOUS!!!

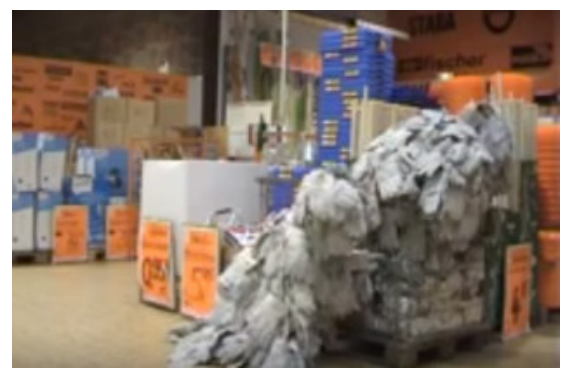

Fig. 1 How to shopdrop yourself looking like a pile of work gloves (https://www.youtube.com/watch? $=b j L C 6 J v i Z t E ;$ accessed 27/02/18).

When I clicked on the video of two people looking like a heap of gloves-well, that just made my day (see Fig. 1). In a very funny way, it brought home the value of engaging experimentally with the worlds of shopping that surround us. These creative interventions are what this article is about. It is about shopdropping.

\section{Creative interventions into con- sumption cultures}

Shopdroppers practise a kind of reverse shoplifting in that they "covertly" place "objects on display in a store" (Watkins-Hughes 2004). The practice has been documented in blog posts, in videos, on websites (cf. Buettemeyer 2007; Konsumpf 2010; TheYesLab 2011; SHIFT! 2012; Cook et al. 2013; Cantrell 2015; TEDx Talks 2015) and in two recently published book chapters (cf. Blas 2013; Cook et al. 2017). Objects as diverse as fruit, shampoo bottles, electronics and, as seen above, work gloves are being shopdropped. As a tactic used „for public art, to promote political views or advertise your services“ (Buettemeyer 2007), shopdropping is done by consumeractivists, factory workers and artists alike. There are numerous examples of messages being smuggled out of factories and warehouses-secretly hidden, for example, in the pockets of jeans (cf. Cook et al. 2013). Shopdroppers are looking to confront consumers with conditions of production, thus lifting the veil of global market relations (cf. Harvey 1990). Many of them find creative ways in which to work through rather than about things, altering the very materiality of the things their protest targets. Shopdropping has been celebrated as an artistic intervention that has the potential to make people reflect on the things they buy (cf. TEDx Talks 2015). It does so in a non-didactic and dialogical 
way that refrains from communicating educational and condescending messages to consumers (cf. Verson 2007).

\section{A cultural geography of shop- dropping}

I find shopdropping a rich practice to work with, both as an activist and as a researcher in human geography. It foregrounds a couple of topics that are currently debated within the social sciences in general and cultural geography in particular. Shopdropping becomes my object of inquiry as well as the vehicle through which I learn and think. My arguments revolve around my own experience of shopdropping a feminist sloth sticker on a diet magazine in a supermarket. I open the discussion by briefly situating shopdropping within the literature around material cultures, disobedient objects and cultural activism. I, then, go on to recount how I came to research my own shopdrop, thus highlighting the value of practice-based and autoethnographic approaches. Next, I introduce three ideas that came out of my creative intervention. First, I argue that a focus on place makes it possible to understand shopdropping as a highly contextualised and ethnographic practice that has the power to subvert the place of the supermarket. Second, I hold that a movement-centred approach draws out the fact that shopdropping is a practice that travels online and offline. Third, I contemplate the question of whether shopdropping can effect positive change, thereby thinking through the connections of geography, art and activism. To conclude, I offer a critical commentary on shopdropping's geographical imaginaries and the forgotten places it produces.

\section{Geography, materiality, culture}

In recent years, geography has seen an increasing number of articles and book chapters published on unruly, disobedient, active and acting things. Shopdropping taps into this growing interest in material geographies (cf. Jackson 2000; Anderson and Tolia-Kelly 2004; Anderson and Wylie 2009; Cook and Tolia-Kelly 2010; Crang 2013).

\section{Material cultures and disobedi- ent objects}

Cultural geographers have (re-)discovered a strong interest in the materiality of the world and in the "stuff" that our lives, natures and cultures are made of (Crang 2013: 276). Scholars of material cultures set out to rethink the relations between people, places and things. They wish to think with rather than about things (cf. Turkle 2007; Cook and Woodyer 2012). Sherry Turkle (2007), in collecting autobiographical stories of people and their things, shows how objects shape people's lives beyond their intentions or conscious 
knowledge. Ian Cook and Tara Woodyer (2012) stress the importance of things in imagining what the world is like; they hold that it is through things that people imagine the world and its geographies. These and other accounts of material culture seek to rethink the nature of materiality. Geographers have drawn on new materialist theorists Jane Bennett (2010) and Donna Haraway (2016), among others, to grapple with this issue. Theorisations of thingness as "unmediated, static physicality" (Anderson and Tolia-Kelly 2004: 670) are no longer inert, passive, acted upon, stable or discrete. New materialist scholars and geographers have come to understand things as lively, active, acting, and vibrant (cf. Bennett 2010). One strand of material geographical research that appreciates the liveliness of things is the body of work on subversive material cultures. Disobedient things are receiving growing attention in accounts of political movements (cf. Bartlett 2016; Yara and Karakayali 2017) and commodity chain activism (cf. Mukherjee and Banet-Weiser 2012; Cook et al. 2013). London's V\&A Museum held an exhibition on Disobedient Objects in 2014/15, showcasing pieces from a diverse range of social and political movements (cf. Flood and Grindon 2014). This is where shopdropping and shopdropped things come into play. The literature on subversive material cultures allows to conceptualise shopdropping as a contentious political

practice that materialises dissent through the very objects it contests.

\section{Geography, art and activism}

My thoughts on shopdropping are largely influenced by recent conversations in geography, art and activism. There is a growing body of literature on artistic and creative geographies (cf. Madge 2014; Hawkins 2015) and on the political value of creative geographical interventions (cf. Marston and De Leeuw 2013; De Leeuw and Hawkins 2017). These literature is closely associated with accounts of making and crafting (cf. Gauntlett 2011; Carr and Gibson 2016). What I would like to draw out here is that arts and crafts have not only been of interest as an object of inquiry but also as a means of doing research. Geographers have taken to use creative practices as research methods. At the centre of these encounters is the insight that valuable ideas can come out of ,just doing something. Doings and makings have a value in themselves that is often overlooked in a focus on output (cf. Hawkins 2015: 263). Practice-based research generates ,knowledge from within particular practices and experiences“ (Banfield 2016: 463) and, therefore, requires a certain openness to the process of research (cf. Foster and Lorimer 2007: 426). What has inspired me in particular is that geographers have drawn on practice-based methods to think about geographical and other concepts (cf. Hawkins 2011: 473). This is 
what I am aspiring to do here. Through shopdropping, I wish to think about the cultural geographies of this practice. In the next section, I expand on how I think a practice-based and autoethnographic approach helps me to do that.

\section{Practice-based and autoeth- nographical research}

Why did I decide to do shopdropping myself? I could have provided an analysis of shopdrops as documented by other activists. There are loads of them online. But I opted to do it by myself instead. There are two reasons for this decision: First, I see a strong value in autoethnographic and practice-based approaches and their focus on openness and process. The second reason is that I badly wanted to get away from my computer. At the time of writing this text, I was doing my master's and was spending around 90 percent of my working time at my desk. I was dying to get ,out there' and ,do something.

\section{Here is what I did...}

I started to research shopdropping on the internet and quickly found inspiring, hilarious, bewildering, and absurd examples (remember the work gloves in Fig. 1). I began to think about what kind of shopdrop I wanted to do by myself. I quickly settled on doing a feminist drop as I am constantly angered by how women, their bodies and sexualities are being represented in adverts and on packaging of consumer goods. So what did I do? I did not do an elaborate artwork. To be honest, I simply photocopied a feminist sloth sticker my sister Mona had given to me last Christmas. It was cute. And it had a radical message: "Riots not Diets“ (see Fig. 2). I, then, ventured off, sticker concealed in my pocket, to do the drop in a supermarket in my neighbourhood. But I will get to that more detailed later. I used a field diary and a digital camera to document and reflect on my practice. Photos, screenshots and short vignettes of my experience are weaved into this text. Autoethnography played an important role in theorising what I was doing. As a methodological tool, it strings together auto (the self), ethno (culture) and graphy (research and writing) (cf. Chang 2008: 48). Its analysis is based on carefully documenting and reflecting on one's own experiences. However, autoethnography is more than mere self-narrative or academic navelgazing. Through narration, it places the self in a broader cultural context and aims at understanding how personal experiences relate to wider contexts (cf. Butz 2010: $138,141)$. Geographers have explored a range of topics through autoethnographic engagements such as landscape (cf. Wylie 2005), harassment (cf. Valentine 1998) and archiving (cf. DeLyser 2015). With the following analysis, I wish to contribute to these accounts. Shopdropping the sloth 
sticker made me reflect on the ways in which this practice manages to politicise places of consumption, on how it is situated in wider activist networks and on how it manages to make change.

\section{Things out of place}

Shopdropping plays with things in places. It engages people, customers, consumers. Catching them off guard, making them wonder. I argue that an approach orientated towards the concept of place is insightful in two ways: First, shopdropping is a placebased and ethnographic practice that engages thoroughly with the materiality of a certain context. Second, it jams commodity culture by revealing the politics at work within places of shopping.

\section{An ethnographic practice}

Alright, I'm doing it! I'm in the supermarket. Really excited! I've come to shopdrop something I made. Well, not exactly made. I've photocopied the feminist sloth Mona gave to me for Christmas. I sneak through the aisles. Okay, quick! I grab one of the diet magazines from the stand, quickly take out the sticker from my coat pocket and stick it on top. I put the magazine back on the stand - done! Wow! Now, let's get out of here...
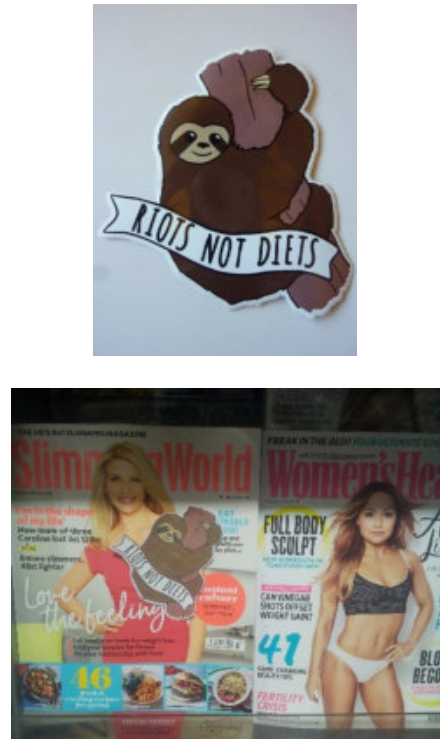

Fig. 2 Before and after - Shopdropping a feminist sloth sticker on a diet magazine in a supermarket (author's own photograph).

I did it. I shopdropped a feminist sloth sticker (see Fig. 2). I am now officially among the group of "guerrilla counterconsumerists" (SHIFT! 2012). And it feels good! It took me a couple of days to figure out where and how to do the drop. You cannot just shopdrop anything anywhere. It takes time to prepare. I needed to think about the product I wanted to target and the message I was hoping to get across. I was sitting at my desk at home, thinking hard, when my eyes caught the sloth sticker on my shelf. I had glued it there when I moved in. The sloth was a feminist one, 
carrying the message "Riots not Diets“. That's it, I thought. Riots not Diets! Just the week before, I had gotten extremely cross with the fact that the supermarket in my neighbourhood sold a whole range of women's slimming and fitness magazines. This was the moment to act. Together with the feminist sloth, I would finally show everyone what I thought about these despicable magazines...

What I did on that day was to plan my shopdrop. I was thinking in detail about which product I was going to work with and where to work with it. This is why shopdropping has been called a highly contextualised and „ethnographic" (SHIFT! 2012) practice: It means that activists engage in depth with the places and things that their drop involves. Shopdroppers work through things. They express criticism of material culture through material culture. In the case of my feminist sloth sticker, I was prompted by problematic mass media body politics. I could have written a letter of complaint to the editors of the diet magazine. However, I decided to express my criticism through the very object I despise. The magazine itself became my vehicle of protest; it became materialised dissent (cf. Taws 2014).

\section{Materialising dissent, politicising places}

Shopdroppers tailor their action to the site of the drop. However, for people who find these objects, they can seem strangely out of place. I have no idea what happened after I dropped the feminist sloth. I like to think that someone took the magazine from the stand, looked at it, wondered what was, wrong' with it and then, maybe, just maybe, thought about its message. Small acts of twisting popular mass culture like shopdropping have become known as "culture jamming" (Watkins-Hughes 2004). They take culture not to be a totalising force but as a site of contention; something that may be subverted and appropriated (cf. Friesinger et al. 2010: 9). The great thing about shopdropping and culture jamming is that it works in places of consumption. The supermarket with its slimming magazines represented to me a place in which misogynistic body politics were being promoted. Through my shopdrop, however, it became a place where these body politics could be contested. Shopdropping allows to reveal the politics at work within places of consumption that would otherwise remain largely invisible.

\section{Moving things, moving practices}

Shopdropping is a highly contextualised and ethnographic practice that has the potential to subvert commodity cultures. The next section approaches shopdropping from a different geographical angle: namely as a practice in motion. It highlights the mobility of shopdropped things and of the practice of shopdropping itself. 


\section{Shopdropping goes travelling}

The journeys of things have been discussed at length in geography, among others in the growing field of follow-the-thing research (cf. Cook et al. 2004; Cook and Harrison 2007; Gregson et al. 2010). However, commodities are not the only things that travel. Shopdropped things do, too. In my case, I got a sloth sticker for Christmas in Germany. I took it to Exeter, where I did my master's, and stuck it on the wall of my room. A couple of months later, I photocopied the very same sticker and dropped it onto a magazine. A movement perspective can provide us with a deeper engagement of where objects come from, where they go to and which relations they form underway (Cook and Woodyer 2012: 227).

Shopdropped things travel and so does the practice of shopdropping. Activists share ideas on websites and blogs (see www. instructables.org; www.detructables.org and an example of their work in Fig. 3) as well as with friends and fellow shopdroppers. They make the practice travel across online and offline worlds. I, for example, browsed the internet to get ideas before I dropped the sloth. There are tons of funny and exciting objects people have shopdropped. One of the things I found was the work of the Craftivist Collective who secretly slip messages into the pockets of trousers and skirts in clothing stores (see https://craftivist-collective.com and an example of their work in Fig. 4). This and other examples inspired me to think about what I wanted my own shopdrop to be like. I was becoming so enthusiastic that told my friends over coffee what I was thinking of doing. They, in turn, told me about hilarious shopdropping examples they had come across with which I, again, looked up online. This illustrates how the practice of shopdropping travels widely, online and offline, from internet user to internet user, friend to friend, activist to activist.

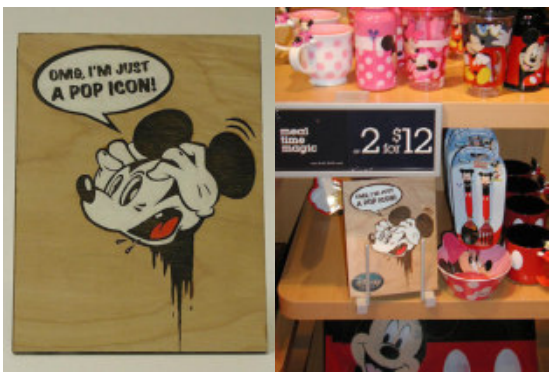

Fig. 3 A Mickey Mouse sign shopdropped in a Disney store (http://www.instructables.com/ id/How-To-Shop-Drop/; accessed 27/02/18).

\section{Does it work?}

Did the sloth sticker change anything? I like to think that it did. It is a tricky question whether shopdropping actually works. In this last section, I tend to two points: First, I briefly draw out the supposed culture/ politics dichotomy inherent in critiques 
of cultural activism. Second, I comment on the ongoing debates on the value of creative interventions and discuss how I feel shopdropping manages to make a change-one sloth sticker at a time.

\section{Can culture be political?}

There are a number of things I have heard people say about cultural activism over the years. One recurring criticism is that it could not be political-it is said to be, after all, an intervention into culture and not into politics. This critique is echoed in the representation of cultural activism as soft, low-key and easy. The Craftivist Collective, for example, advocates for the „art of gentle protest“ (Craftivist Collective 2017). It often seems that cultural activism is perceived as opposed to supposedly radical or proper activism. What speaks from this idea is that, first, acts of protest are being categorised into either cultural or political actions and that, second, these categories are attributed with a supposed level of radicalness and effect. I think that the labelling and hierarchizing of contentious practices is deeply problematic. It supposes that there is one right way to make a change. This view has been challenged by Jennifer Verson who writes for the activist group The Trapese Collective. She argues that we should embrace the idea of a "full spectrum resistance“; after all, „who can really know what it is that really inspires an individual to care, or to turn away, to give up or to rise up?" (Verson 2007: 171) Verson plays to the fact that it is difficult to measure how activism works and what it does.

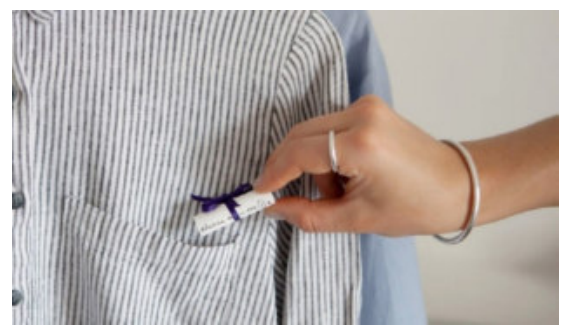

Fig. 2 Mini Fashion Statements by the Craftivist Collective (https://craftivist-collective. com/Projects/Mini-Fashion-Statements; accessed 01/03/18).

\section{The æffects of shopdropping}

Shopdropping might have been called low-key, easy, gentle and funny. Fine. It often is low-key, easy, gentle and funny. But that does not mean that it cannot be radical or make a change. With shopdropping, consumer activists have developed a unique way to draw attention to the fact that commodity culture is always already political and that it can be acted upon (Mukherjee and Banet-Weiser 2012: 4-5). Louise Ashcroft, a London-based artist and passionate shopdropper, tells us that her actions are geared towards making shoppers feel 
"powerless“ and „liberated“ at the same time (We make money not art, 2017): Powerless in the face of mass culture's hegemony and liberated by the insight that commodity culture can nevertheless be subverted. Ashcroft's thoughts point to a broader debate around the effects of activist art. Stephen Duncombe (2016) has offered the concept of æeffect as an intriguing way to think about the myriad ways in which activist art works. According to him, „before we act in the world, we must be moved to act" (Duncombe 2016: 119). Activist art generates both effects, which are discernible changes, and affects, which are changes of mind or emotional setting (Duncombe 2016: 118-119). Æffect comes in different shapes, for example in building communities (of shopdroppers all over the world) or in altering perception (of diet magazines) (Duncombe 2016: 120-125). I do not know for sure that anyone saw or was moved by my sloth sticker. What I know, however, is that it made a difference for me. Preparing, doing, thinking through and writing about the shopdrop made me realise how powerful small acts of twisting consumption culture can be. It made me hopeful that we do not need to accept things as they are-such as the horrific body politics of diet magazinesbut that we can engage with and change them.

\section{Conclusion: Forgotten places}

Shopdropping as a cultural and creative practice materialises protest at the very heart of commercial material culture. Thinking through its materialities and politics, a cultural geography perspective offers three intriguing insights. I highlighted, first, that shopdropping is an ethnographic and subversive practice, second, that it is a practice on the move and, third, that it has the power to produce æffect. In the few remaining lines I point out the geographical imaginaries and blind spots within shopdropping.

\section{Which things cannot be shop- dropped?}

Objects dropped in stores have the power to make customers wonder and think. Messages shopdropped by factory workers make visible that products are made somewhere by someone. In this regard, shopdropping can be effective in, at least partly, lifting the veil that obscures how, by whom and under which conditions the products we buy are made (cf. Harvey 1990). This is important. But doing shopdropping myself made me think about another question: Which products can be dropped? Which cannot? In most, if not all, cases, shopdropping works with manufactured goods. I have come across examples of messages dropped into/with clothes, shoes, handbags, mobile phones, advent calendars, tins, and 
shampoo bottles. I have not come across, for example, shopdropped cocoa beans, bananas or pineapples-or any other agricultural produce. I have the feeling that this generates an uncomfortable silence on agricultural products and the no less scandalous working conditions on farms. I am interested in how these forgotten places could be tended to through shopdropping or whether we need other activist practices to make them visible.

\section{Geographical imaginaries}

My second point concerns the geographical imaginaries at play within shopdropping. If we take seriously that geographies are imagined through things, then I wonder which geographies are imagined through shopdropped items. I would argue that we imagine a linear value chain that directly connects producers and consumers. How else would someone find a secret letter in the pocket of their jeans? It must have travelled more-or-less straight from the factory to the customer. I think that this geographical imaginary can become problematic. It comes quite close to the managerialist perspective of supply chain management that imagines commodities as following linear, monitored trajectories. What really happens, however, is that things frequently get lost, travel elsewhere or cannot be found anymore (cf. Hulme 2017). Re-thinking the spatial imaginaries at play in shopdropping is an interesting way forward in this discussion. Until then, I would like to close with a quote for all practising and aspiring shopdroppers: „Culture jamming is useless fun. That's exactly why you should do it." (Verson 2007: 178)

\section{ZUR AUTORIN}

Mara Mürlebach fragt sich, seitdem sie alleine einkaufen geht, woher Konsumgüter kommen und wer sie unter welchen Bedingungen herstellt. Zurzeit studiert sie im Master Critical Human Geographies an der University of Exeter (UK). Aus feministischer Perspektive forscht sie zu globalen Wertschöpfungsketten, Konsumkulturen und aktivistischen Praktiken. Außerdem möchte sie mehr über kreative und kollaborative Forschungsansätze erfahren. Das feministische Faultier aus diesem Artikel wohnt weiterhin auf dem Regal über ihrem Schreibtisch.

Der Beitrag wurde von Clément Dréano und Markus Kohlmeier gereviewed und von beiden redaktionell betreut. Tatiana Huppertz, Tanja Strukelj und Andreas Schulz haben den Artikel lektoriert.

\section{REFERENCES}

Anderson, Ben/Tolia-Kelly, Divya (2004): Matter(s) in social and cultural geography. In: Geoforum, Vol. 35/6, p. 669-674. 
Anderson, Ben/Wylie, John (2009): On geography and materiality. In: Environment and Planning A, Vol. 41/2, p. 318-335.

Banfield, Janet (2016): Knowing between: generating boundary understanding through discordant situations in geographic-artistic research. In: Cultural geographies, Vol. 23/3, p. 459-473.

Bartlett, Alison (2016): Sites of feminist activism: Remembering Pine Gap. In: Continuum: Journal of Media and Cultural Studies, Vol. 30/3, p. 307-315.

Bennett, Jane (2010): Vibrant matter: a political ecology of things. Durham: Duke University Press.

Blas, Zach (2013): GRIDs, Gay Bombs, and Viral Aesthetics: Queer Technologies' Networked Assemblages. In: Keilty, Patrick/Dean, Rebecca (eds.): Feminist and Queer Information Studies Reader. Sacramento: Litwin Books, p. 662-678.

Buettemeyer, Marcus (2007): Shopdropping. In: Urban Dictionary, 25/12/2007. Online available at https://www. urbandictionary.com/define.php?term=shopdropping (26/02/2018).

Butz, David (2010): Autoethnography as sensibility. In DeLyser, Dydia/Herbert, Steve/Aitken, Stuart/Crang, Mike/McDowell, Linda (eds.): The SAGE Handbook of Qualitative Geography. Los Angeles: Sage, p. 138-155.

Cantrell, Amanda (2015): Artists drop while they shop: „Shop droppers" alter packaging of retail goods to spread messages. In: CNN Money, 20.07.2015. Online available at: http://money.cnn.com/2005/07/19/news/funny/shopdropping/ (21.02.2018).

Carr, Chantel/Gibson, Chris (2016): Geographies of making: Rethinking materials and skills for volatile futures. In: Progress in Human Geography, Vol. 40/3, p. 297-315.

Chang, Heewon (2008): Autoethnography as method. Walnut Creek: Left Coast Press.

Cook, Ian et al. (2004): Follow the thing: Papaya, In: Antipode, 36/4, p. 642-664.

Cook, Ian/Harrison, Michelle (2007): Follow the Thing: West Indian Hot Pepper Sauce. In: Space and Culture, Vol. 10/1, p. 40-63.

Cook, Ian/Tolia-Kelly, Divya (2010): Material geographies. In Hicks, Dan/Beaudry, Mary (eds.): The Oxford
Handbook of Material Culture Studies. Oxford: Oxford University Press, p. 99-122.

Cook, Ian/Woodyer, Tara (2012): Lives of things. In: Barnes, Trevor/Peck, Jamie/Sheppard, Eric(eds.): Wiley-Blackwell companion to economic geography. Oxford: Wiley-Blackwell, p. 226-241.

Cook, Ian et al. (2013): The 17 best examples of shop-dropping... ever. In: followthethings.com, 22.03.2013. Online available at: https://followtheblog.org/2013/03/22/paperactivism-in-store-in-things-on-things/ (15.01.2018).

Cook, Ian et al. (2017): followthethings.com: Analysing Relations between the Making, Reception and Impact of Commodity Activism in a Transmedia World. In Söderström, Ola/Kloetzer, Laure/Jeannerat, Hugues (eds.): Innovations sociales: comment les sciences sociales transforment la société. Neuchâtel: Université de Neuchâtel, p. 50-61.

Craftivist Collective (2017): Craftivism works! WWF used our 'gentle protest' approach and won, In: Craftivist Collective, 26.07.2017. Online available at: https://craftivist-collective.com/WWF-gentle-protest-campaign-success (27.02.2018).

Crang, Philip (2013): Material geographies. In: Crang, Philip/Goodwin, Mark/Cloke, Paul (eds.): Introducing Human Geographies. London: Hodder Arnold, p. 276-291.

De Leeuw, Sarah/Hawkins, Harriet (2017): Critical geographies and geography's creative re/turn: poetics and practices for new disciplinary spaces. In: Gender, Place and Culture, 24/3, p. 303-324.

DeLyser, Dydia (2015): Collecting, kitsch and the intimate geographies of social memory: a story of archival autoethnography. In: Transactions of the Institute of British Geographers, Vol. 40/2, p. 209-222.

Duncombe, Stephen (2016): Does it work? The æffect of activist art. In: social research, Vol. 83/1, p. 115-134.

Flood, Catherine/Grindon, Gavin (2014): Disobedient Objects. Published to accompany an exhibition held at the Victoria and Albert Museum, 26/07/2014-01/02/2015, London: V\&A Publishing.

Foster, Kate/Lorimer, Hayden (2007): Cultural geographies in practice: Some reflections on art-geography as collaboration. In: Cultural geographies, Vol. 14/3, p. 425-432. 
Friesinger, Günther/Grenzfurthner, Johannes/Ballhausen, Thomas (2010): Welcome to the Battlefield. Please Make Yourself Comfortable. In Friesinger, Günther/Grenzfurthner, Johannes/Ballhausen, Thomas (eds.): Urban Hacking: Cultural Jamming Strategies in the Risky Spaces of Modernity. Bielefeld: transcript, p. 9-12.

Gauntlett, David (2011): Making is Connecting: The social meaning of creativity, from DIY and knitting to YouTube and Web 2.0. Cambridge: Polity Press.

Gregson, Nicky/Crang, Mike/Ahamed, Farid Uddin/ Akhter, Nasreen/Ferduos, Raihana (2010): Following things of rubbish value: End-of-life ships, 'chock-chocky' furniture and the Bangladeshi middle class consumer. In: Geoforum, Vol. 41/6, p. 846-854.

Haraway, Donna (2016): Staying with the Trouble: Making Kin in the Chthulucene. Durham: Duke University Press.

Harvey, David (1990): Between Space and Time: Reflections on the Geographical Imagination. In: Annals of the Association of American Geographers, Vol. 80/3, p. 418-434.

Hawkins, Harriet (2011): Dialogues and Doings: Sketching the Relationships Between Geography and Art. In: Geography Compass, Vol. 5/7, p. 464-478.

Hawkins, Harriet (2015): Creative geographic methods: knowing, representing, intervening. On composing place and page. In: Cultural geographies, Vol. 22/2, p. 247-268.

Hulme, Alison (2017): Following the (unfollowable) thing: methodological considerations in the era of high globalisation. In: Cultural geographies, Vol. 24/1, p. 157-160.

Jackson, Peter (2000): Rematerialising social and cultural geography. In: Social and Cultural Geography, Vol. 1/1, p. 9-14.

Konsumpf (2010): Shopdropping - Subversive Konsumunterwanderung. In: Konsumpf, 09/11/2010. Online available at: http://konsumpf.de/?p=8697 (16.05.2018).

Madge, Clare (2014): On the creative (re)turn to geography: poetry, politics and passion. In: Area, Vol. 46/2, p. 178-185.

Marston, Sallie/De Leeuw, Sarah (2013): Creativity and geography: toward a politicized intervention. In: The Geographical Review, Vol. 103/2, p. iii-xxvi.

Mukherjee, Roopali/Banet-Weiser, Sarah (2012): Commodity Activism: Cultural Resistance in Neoliberal Times.
New York: New York University Press.

SHIFT! (2012): How to shop drop, In: Instructables. Online available at: http://www.instructables.com/id/ How-To-Shop-Drop/ (28.02.2018).

Taws, Richard (2014): Exhibition Review: Disobedient Objects at the Victoria and Albert Museum London. In: West 86th: A Journal of Decorative Arts, Design History, and Material Culture, Vol. 21/2, p. 297-300.

Louise Ashcroft (2015): Shopping and subversion. TEDxHackneyWomen, 23.06.2015. Online available at: https:// www.youtube.com/watch?time_continue $=3218 v=$ AYe3VjemYP4 (21.02.18).

TheYesLab (2011): Shop Dropping Product Labels. In: The Yes Lab, 07/07/2011. Online available at: http://destructables.org/destructable/shop-dropping-product-labels-yeslab (21.02.2018).

Turkle, Sherry (2007): Evocative objects: things we think with. Cambridge: MIT Press.

Valentine, Gill (1998): „Sticks and stones may break my bones": A personal geography of harassment. In: Antipode, Vol. 30/4, p. 305-332.

Verson, Jennifer (2007): Why we need cultural activism. In: The Trapese Collective (eds.): Do it yourself: a handbook for changing our world. London: Pluto Press, p. 171-186.

Watkins-Hughes, Ryan (2004): Shopdrop. In: Shopdropping.net. Online available at: http://shopdropping. net/ (26.02.2018).

We make money not art (2017): Vegetable smuggling, grimy goods and other retail sabotages. An interview with Louise Ashcroft. In: We-make-money-not-art.com, 23.08.2017. Online available at: http://we-make-money-not-art.com/ vegetable-smuggling-grimmy-goods-and-other-retail-sabotages-an-interview-with-louise-ashcroft/ (21.02.2018).

Wylie, John (2005): A single day's walking: narrating self and landscape on the South West Coast Path., In: Transactions of the Institute of British Geographers, Vol. 30/2, p. 234-247.

Yara, Özge/Karakayali, Serhat (2017): Emergent infrastructures: solidarity, spontaneity and encounter at Istanbul's Gezi Park uprising. In: Brown, Gavin/Feigenbaum, Anna/ Frenzel, Fabian/McCurdy, Patrick (eds.): Protest camps in international context. Bristol: Policy Press, p. 53-69. 


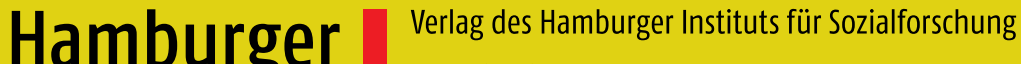 Edition}

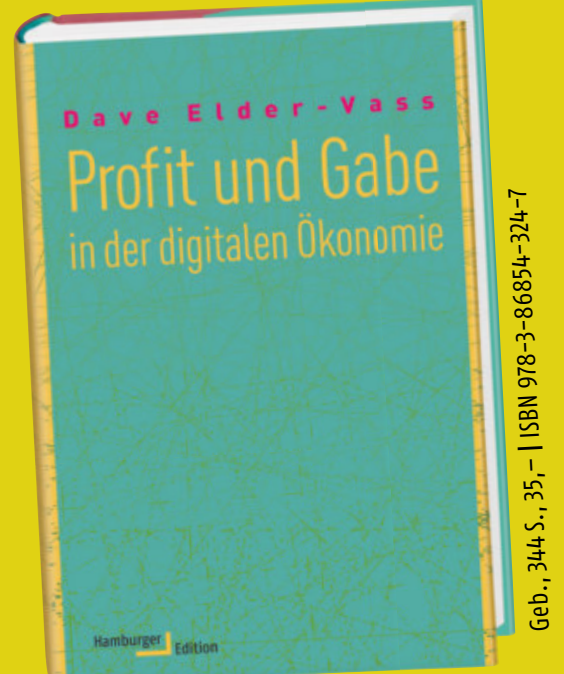

Zygmunt Bauman Leben als Konsum

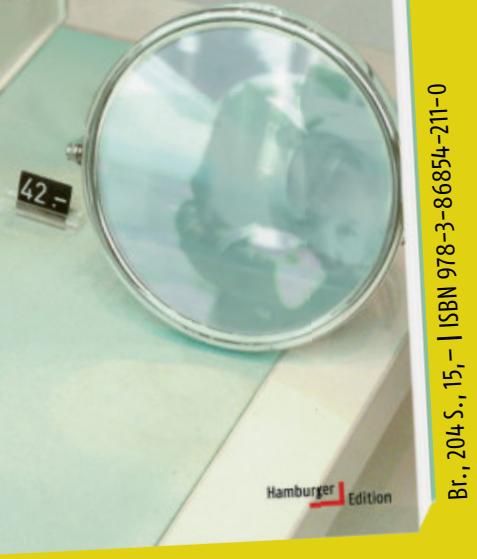

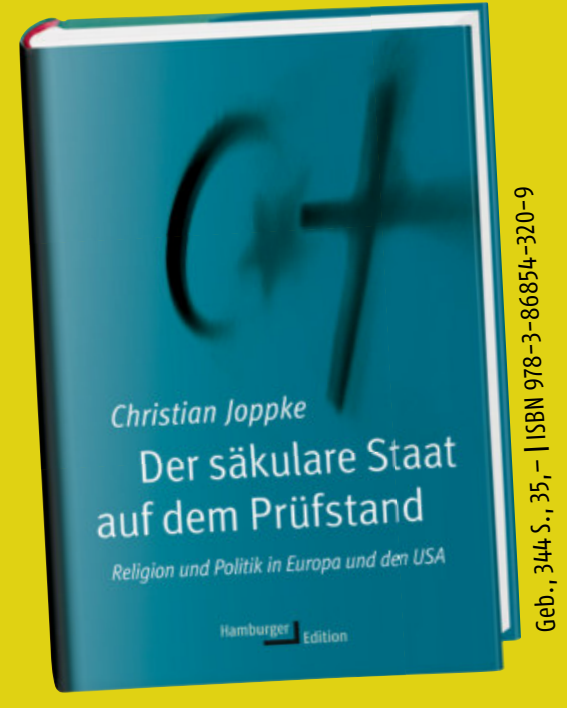

Alle Titel sind auch als E-Book erhältlich.

Stephanie DeGoover I Alostair Hunt lida Maxwell I Samuel Moyn

Vom Recht, Rechte zu haben

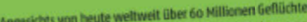
Angesidus we hishe kassene wen Hannah arendt, dass es sa

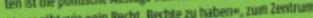

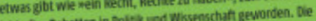

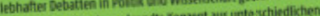

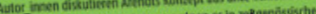

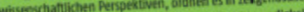
und politische pebatten ein und betones die Notwendigke:

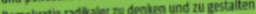

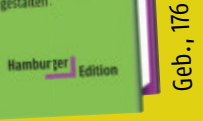

Leseproben und Bestellungen:

hamburger-edition.de 


\section{Die gemeinschafts- bildende Funktion des Konsumierens \\ bei Marx, Bourdieu und Laclau/Mouffe}

Der Artikel gibt einen Überblick über den Zusammenhang zwischen Konsum und der Herausbildung politischer Gemeinschaften bei Karl Marx, Pierre Bourdieu und Ernesto Laclau und Chantal Mouffe. Für Marx ist die Konsumption in kapitalistischen Gesellschaften konstiutiv für die Herausbildung des Klassenwiderspruches zwischen Arbeit und Kapital. Auch Bourdieu analysiert die Rolle des Konsumierens in Hinblick auf ihre klassenbildende Funktion. Er wendet sich jedoch von einem rein materialistischen Konsumptionsverständnis ab und betont die symbolisch-kulturelle Dimension des Konsumierens für die Herausbildung von Klassen in modernen Gesellschaften. Anders als Bourdieu geben Laclau/Mouffe einen Ausblick auf die Frage, wie sich unterschiedliche soziale Positionen auf der Grundlage einer Vervielfältigung sozialer Antagonismen zusammenschließen, um neue Gemeinschaftsformen zu bilden.

\section{Schlagwörter}

Kritik; Emanzipation; Gemeinschaft; Postfundationalismus 


\section{Zur Aktualität von Marx, Bourdieu und Laclau/Mouffe}

In der Mitte des 19. Jahrhunderts behandelte Karl Marx mit seinen präzisen Analysen gesellschaftlicher Verhältnisse die drängenden Probleme seiner Zeit. In diesem Jahr, da sich Marx' Geburtstag zum 200. Mal jährt, wird verstärkt auf die Relevanz seiner Theorien für die Krisen unserer Zeit hingewiesen und gezeigt, dass diese hochaktuell sind. Marx kritisiert die Methode der Klassischen Ökonomie, da sie die gesellschaftlichen Akteure danach beschreibe, wie produziert und konsumiert werde, dabei jedoch übersieht, dass die herrschenden Verhältnisse der Produktion, Konsumption und Distribution auch andere sein könnten. „Die Ökonomen“, schreibt Marx „stellen die bürgerlichen Produktionsverhältnisse, Arbeitsteilung, Kredit, Geld etc., als fixe, unveränderliche und ewige Kategorien hin." (Marx 1977: 126) Als fixierte und unveränderliche Kategorien erscheinen diese laut Marx deswegen, weil nicht in den Blick genommen wird, „wie diese Verhältnisse produziert werden, d.h. die historische Bewegung, die sie ins Leben ruft.“ (Marx 1977: 126) Marx' dialektisch-materialistische Methode verdeutlicht, dass die geltenden, von der kapitalistischen Produktionsweise eingerichteten und aufrechterhaltenen Verhältnisse eben nicht sind, was sie vorgeben zu sein - nämlich unabänderlich und ewig.
Im vorliegenden Artikel soll die Aktualität von Marx' Werk in Hinblick auf seine Verknüpfung von Konsumption und der Herausbildung politischer Gemeinschaften, die er in der Form des Klassenwiderspruches Arbeit/Kapital konzipiert, hervorgehoben werden. Bei Marx ist die Art und Weise des Konsumierens, im Sinne einer ökonomisch-materiellen Konsumption, konstitutiv für die Herausbildung der Klassen. Obgleich die Wirkmächtigkeit und Aktualität Marx' Analysen für die Gegenwart keineswegs angezweifelt werden soll, können sie nicht kritiklos auf heutige Verhältnisse angewandt werden. Heutige demokratische Gesellschaften stehen vor anderen Herausforderungen und Möglichkeiten, denen die Theorie des klassischen Marxismus nicht gerecht wird. Einer der interessantesten Marx-Rezipient_innenen in Hinblick auf die gemeinschaftsbildende Funktion des Konsumierens ist der französische Soziologe Pierre Bourdieu, der in den 1970er Jahren beginnt, seine Analysen des sozialen Raumes vorzunehmen. In dieser hebt er die symbolisch-kulturelle Dimension des Konsums für die Herausbildung von Klassen hervor. Klassenwidersprüche lassen sich für Bourdieu nicht mehr auf rein ökonomische Kategorien zurückführen (vgl. Bourdieu 2016b: 31). Stattdessen ist bei Bourdieu der soziale Raum durchzogen von Antagonismen, die sich durch den Konsum symbolischer Güter und der Hervorbringung kulturellen Kapitals, wie 
zum Beispiel Bildung, konstituieren (vgl. Bourdieu 2016a: 41).

Die postmaterialistische Kritik an einem rein ökonomistischen Konsumptionsverständnis, die Bourdieu in den 1970er Jahren formuliert, hat nicht an Plausibilität und gesellschaftlicher Relevanz eingebüßt. Im Gegenteil, wir erleben heute einen enormen Zuwachs der Bedeutung symbolisch-kultureller Konsumption, der maßgeblich mit dem technischen Fortschritt und der Zunahme des Konsums virtueller Güter zusammenhängt. Eine kritische Analyse heutiger kapitalistischer Verhältnisse darf neue Möglichkeiten zur symbolischen Vernetzung und die Bedeutung kultureller Dimensionen für soziale Prozesse nicht unbeachtet lassen. Paradoxerweise jedoch verliert das bourdieusche Begriffsinstrumentarium heute womöglich gerade aufgrund der Betonung des Symbolisch-Kulturellen an Überzeugungskraft. Betrachtet man Unterdrückungsverhältnisse im sozialen Raum jenseits der eurozentristischen Perspektive nationaler und supranationalen Grenzen, wird man unweigerlich auf Kämpfe an der Basis ökonomisch-materialistischer Konsumptions-, Produktions- und Distributionsverhältnisse zurückgeworfen.

Auch die postmarxistische Konzeption der politischen Theoretiker Ernesto Laclau und Chantal Mouffe betonen in Abgrenzung zum ökonomischen Materialismus die
Bedeutung des Symbolischen. Gleichzeitig ermöglicht jedoch ihr Diskursbegriff die Gleichursprünglichkeit symbolischer und materieller Artikulationen zu denken, die ein differentielles System sozialer Positionen bilden (vgl. Laclau/Mouffe 2015: 143). Wie sich differentielle soziale Positionen zusammenschließen, um neue Gemeinschaftsformen im Sinne emanzipatorischer Artikulationen zu bilden, soll nachfolgend in Anschluss an Marx, Bourdieu und Laclau/Mouffe erörtert werden. Dazu werden die Konzepte des Konsumierens sowie der Gemeinschaftsbildung bei Marx, Bourdieu und Laclau/Mouffe dargestellt und miteinander verglichen.

\section{Individuelle und produktive Konsumption bei Marx}

Im fünften Kapitel „Arbeitsprozeß und Verwertungsprozeß“ des Kapitals beschreibt Karl Marx Arbeit als einen Prozess zwischen Mensch und Natur in Form von Konsumption (vgl. Marx 1968a: 192). Arbeit als der Prozess des Verzehrens, Verbrauchens oder Verspeisens von ,stofflichen Elementen" (ebd.) teilt Marx in zwei Formen der Konsumption ein: die individuelle und produktive Konsumption (vgl. Marx 1968a: 596). Die individuelle Konsumption ist die - im Sinne eines Austausches zwischen Menschen und Natur - wiederholte Aufrechterhaltung des Lebens. Der Mensch lebt, weil er arbeitet 
und „seinen Stoffwechsel mit der Natur durch seine eigne Tat vermittelt, regelt und kontrolliert.“ (Marx 1968a: 192)

Die individuelle Konsumption ist also die einfache Unmittelbarkeit der Arbeit, die nichts anderes ist, als der unaufhörlich arbeitende Vermittlungsprozess des sich aufrechterhaltenden Lebens. Der Mensch konsumiert in Form des Atmens (Mensch/ Tat) von Luft (Produkte der Natur) oder des Verdauens (Mensch/Tat) von Nahrung (Produkte der Natur). Indem der Mensch konsumiert, wird er selbst zum Produkt dieses Prozesses: „Das Produkt der individuellen Konsumption ist daher der Konsument selbst.“ (Marx 1968a: 198) In der individuellen Konsumption werden also die „Produkte als Lebensmittel des lebendigen Individuums [...] verzehrt.“ (Marx 1968a: 198)

Das Resultat der produktiven Konsumption hingegen ist nicht einfach die Aufrechterhaltung des Lebens, sondern die Aufrechterhaltung des Lebens als Arbeitskraft. Die Arbeit in ihrer produktiven Dimension ist nicht nur in der Lage, sich selbst als ihr eigenes Produkt zu reproduzieren. Während die Arbeit als individuelle Konsumption, sich selbst als Produkt reproduziert ist „das Resultat der produktiven Konsumption [...] ein vom Konsumenten unterschiedenes Produkt.“ (Marx 1968a: 198) Als produktive Konsumption erschafft sie ein von sich selbst unterschiedenes
Produkt, das distribuiert werden kann. Die produktive Konsumption verzehrt die Produkte als „Lebensmittel der Arbeit, seiner sich betätigenden Arbeitskraft.“ (Marx 1968a: 198) In ihrer Funktion als Arbeitskraft unterscheidet sich die Arbeit als individuelle Konsumption von der produktiven Konsumption, weil sie von anderen konsumiert werden kann.

Ein anschauliches Beispiel für die individuelle Konsumption ist das Essen von Lebensmitteln. Der_die Arbeiter_in isst, um sich am Leben zu erhalten. Durch die produktive Konsumption entsteht ein vom Leben der Arbeiter_innen unterschiedenes Produkt: Die Arbeitskraft, die der_die Arbeiter_in dem_der Kapitalist_in anbietet und mit der er_sie Produkte herstellt, wird dabei von dem_der Kapitalist_in konsumiert. Die Arbeit wird vom sich (re-)produzierenden Leben zum Produkt konsumierbarer Arbeitskraft. Die produktive Konsumption des Arbeiters in den kapitalistischen Produktionsverhältnissen ist zugleich „die Konsumption seiner Arbeitskraft durch den Kapitalisten, der sie gekauft hat." (Marx 1968a: 596) Der_die Arbeiter_in erhält Lohn für seine_ihre am Arbeitsmarkt verwendete Ware, welches er für Lebensmittel benötigt, d.h. für seine individuelle Konsumption. Die individuelle Konsumption der Arbeiter_innen wird so ein bloßer Zusatz des Konsumptionsprozesses. Das Leben ist nicht mehr Zweck an sich, sondern dient den Zwecken des Kapitalismus. 


\section{Die Herausbildung des Zwei- klassenmodells bei Marx}

Obgleich die Unterscheidung zwischen individueller und produktiver Konsumption die komplexe Dialektik von Konsumierten und Konsumierenden nicht erfasst, ist sie bei Marx die Bedingung der Möglichkeit für die Ausbildung der Klassengemeinschaften:

Die produktive und die individuelle Konsumption des Arbeiters sind also total verschieden. In der ersten handelt er als bewegende Kraft des Kapitals und gehört dem Kapitalisten; in der zweiten gehört er sich selbst und verrichtet Lebensfunktionen außerhalb des Produktionsprozesses. Das Resultat der einen ist das Leben des Kapitalisten, das der anderen ist das Leben des Arbeiters selbst. (Marx 1968a: 596f.)

Bei Marx finden wir ein tiefgreifendes Verständnis von Konsumption, das sich keineswegs in der Betrachtung von Konsumstrukturen als bloßen Teil des ökonomischen Kreislaufes erschöpft. Vielmehr begreift er Konsum in seiner strukturierenden Funktion, die die ökonomischen Verhältnisse und die darin wirkenden Akteure und politischen Kämpfe hervorbringt.

\section{Kultureller Konsum und Zersplitterung des Klassen- antagonismus bei Bourdieu}

In seinem Hauptwerk Die feinen Unterschiede (1979) untersucht Bourdieu die Positionen und Relationen des sozialen Raumes, indem er zahlreiche Indizes des kulturellen Konsums heranzieht wie den „Besitz von Platten oder eines Klaviers, Fernsehgebrauch, Besuch von Museen, Ausstellungen, Varieté-Veranstaltungen, Kinobesuch, Mitgliedschaft in einer Bücherei, Anlegen einer Sammlung, Sportbetätigung" (Bourdieu 2016a: 217). Wie schon Marx begreift auch Bourdieu Konsum als konstitutiv für die Herausbildung sozialer Akteure. Unterschiedliche Arten des Konsumierens stellen Beziehungen her, welche Möglichkeiten und Hürden zur Kapitalakkumulation und so Machtverhältnisse und Stellungen innerhalb des sozialen Raums bestimmen. Während Marx jedoch Konsumption in ihrer ökonomisch-materialistischen Dimension begreift, entwickelt Bourdieu ein Konsumverständnis, das nicht nur ökonomisch-materielle, sondern verstärkt auch kulturelle Dimensionen des Konsumierens in den Blick nimmt. In Abgrenzung zu einer klassisch-marxistischen Lesart, in der kultureller Konsum ein Überbauphänomen darstellt, das von einer materiell-ökonomischen Basis determiniert ist, nimmt dieser bei Bourdieu eine zentrale Funktion in seinen Gesellschaftsanalysen ein. Für Bourdieu gewinnen beispielsweise 
Hochschullehrer_innen in modernen, postmaterialistischen Gesellschaften ihre gesellschaftliche Stellung, ihr „Ansehen“, nicht bloß durch ökonomisches, sondern durch kulturelles, soziales und symbolisches Kapital (vgl. Bourdieu 2016a: 212f.). Bourdieu beschreibt Machtverhältnisse und antagonistische Relationen zwischen sozialen Positionen, die sich als ein mehr oder weniger einheitliches Ensemble unterschiedlicher Konsumarten im sozialen Raum verdichten. Er untersucht die ästhetischen Einstellungen oder den Typ der Lektüre (Krimis, Abenteuerromane, Philosophie, Politik, Wirtschaft, etc.) entsprechend dem Bildungsgrad und der zugeordneten Schicht der Proband_innen und zeigt, wie durch unterschiedliche Geschmacksurteile hierarchische Relationen produziert werden (vgl. Bourdieu 2016a: 70ff., 108ff., 200ff.).

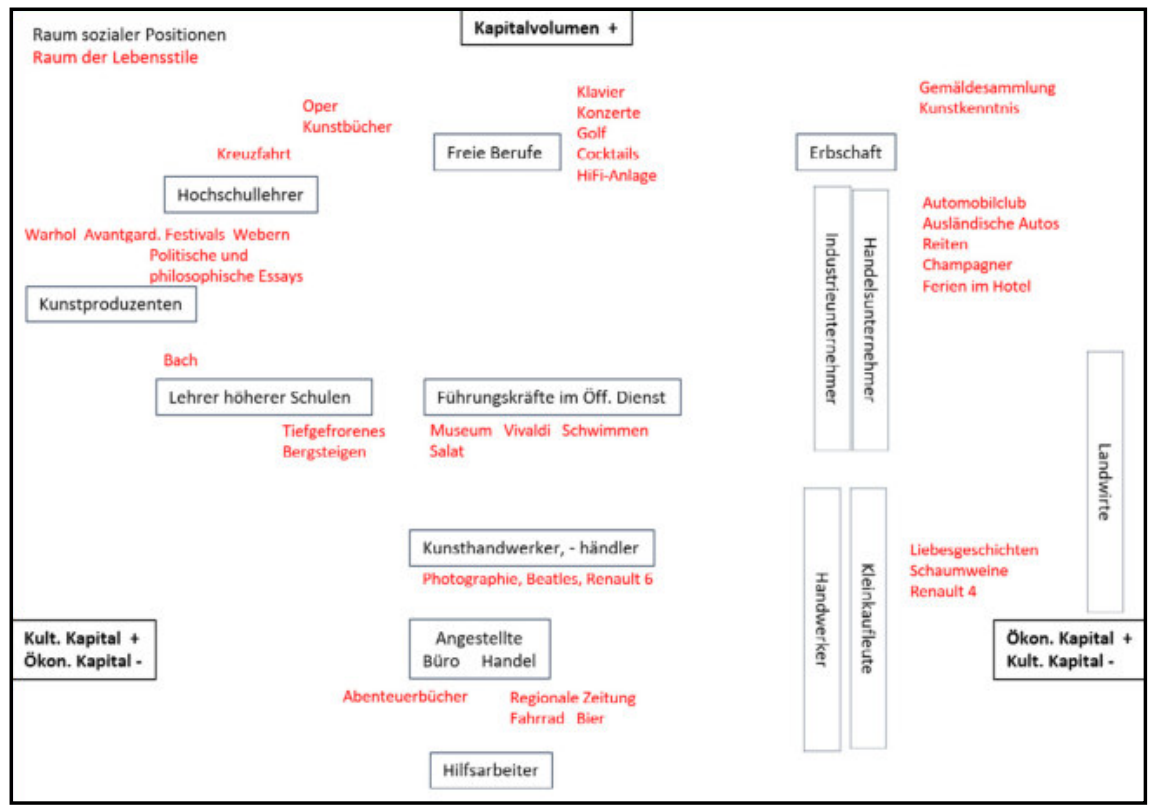

Abb. 1 Der Soziale Raum bei Bourdieu - Soziale Positionen und Lebensstile. Nachbildung eines Diagrammes aus „Die feinen Unterschiede“ (vgl. Bourdieu 2016a: 212f.). 
Das traditionelle Zweiklassenmodell zerfällt bei Bourdieu in mannigfache Positionen, die sich jeweils durch klassenspezifische Habitusformen auszeichnen. Nicht nur ökonomisches Kapital spielt in modernen Gesellschaften eine Rolle für die Zuordnung sozialer Positionen, die Verteilung von Klassenpositionen im Raum des Sozialen Raum erfolgt maßgeblich durch Unterschiede in Bezug auf Vorlieben und Lebensstile.

Nun könnte man die Zunahme der Bedeutung kultureller Konsumgüter, die Bourdieu aufzeigt, auch als einen Hinweis auf die allumfassende Durchdringung des Kapitalismus in modernen westlichen Gesellschaften verstehen. In modernen Kapitalismus konsumiert und produziert jede_r für sich, um sich selbst zu optimieren. Die Klassenkämpfe werden in heutigen Gesellschaften jedoch nicht nur auf einer ökonomischen Eben ausgetragen, sondern durchdringen auch das Symbolische. Vereinzelte Individuen versuchen durch Umstellungsstrategien ihre soziale Position zu verbessern (vgl. Bourdieu 2006a: 227), indem sie Kapital auf den unterschiedlichsten Ebenen (materiell, physisch, geistig, etc.) akkumulieren. Dafür werden Dinge konsumiert, die nicht im Dienste der Aufrechterhaltung und Verbesserung menschlicher Lebensbedingungen stehen, sondern dazu dienen, einen sinnentleerten Prozess des Produzieren, Konsumierens und Distribuierens immer wieder aufs
Neue voranzutreiben. Das Leben des Menschen ist dabei zweitrangig, schlimmer noch, es wird für diesen sinnentleerten Kreislauf ausgebeutet, missbraucht und geopfert.

Man muss kein überzeugter Marxist sein, um sich die Frage zu stellen, ob aktuelle Unterdrückungsverhältnisse allein mit Indikatoren wie der Häufigkeit von Theater- oder Museumsbesuchen oder mithilfe der Frage nach dem Besitz einer Automobilmarke oder dem einen oder anderen Zeitungsabonnement messbar sind. Womöglich verliert eine Analyse kulturellen Konsums und daraus resultierender Unterdrückungsverhältnisse heute an Glaubwürdigkeit. Es vergeht im öffentlichen Diskurs Europas zurzeit kein Tag, in dem das Schützen von Menschenleben und das Schützen vermeintlich kulturellen europäischen Kapitals nicht gegeneinander abgewogen wird.

In weiterer Folge soll die Bedeutung der Marxschen Gesellschaftsanalysen hervorgehoben und ein Ausblick auf ein postmarxistisches Gemeinschaftsverständnis in Anschluss an Ernesto Laclau und Chantal Mouffe gegeben werden. Obgleich die hier angeführte Kritik an Bourdieu angesichts heutiger Krisen notwendig erscheint, ist sie weit davon entfernt, die Bedeutung des kulturellen Konsums und seine gemeinschaftsbildende Funktion in modernen Gesellschaften zu leugnen. Das 
Konsumverständnis Bourdieus, das über die ökonomische Sphäre hinausgeht, skizziert eine breite Verstreuung von Klassenpositionen im sozialen Raum. Die Vielfalt unterschiedlicher sozialer Positionen sollte nicht ausschließlich als Ausdruck einer abzulehnenden kapitalistischen Superstruktur verstanden werden, sondern als Bedingung für demokratisch-pluralistische Gesellschaften. Diese - so soll argumentiert werden - gilt es nicht einfach umzustürzen, sondern vielmehr zu erhalten und radikal zu vertiefen.

Obgleich es falsch wäre, Bourdieus Verweis auf die Streuung der Klassen im sozialen Raum abzulehnen, soll gezeigt werden, dass Bourdieu es verabsäumt zu zeigen, wie sich unterschiedliche soziale Positionen bündeln und neue emanzipatorische Gemeinschaftsformen möglich werden. Bevor diese Argumentation weitergeführt wird, die mit der Frage nach dem emanzipatorischen Potenzial der bourdieuschen Analysen zusammenhängt, soll im Folgenden noch einmal auf das Marxsche Zweiklassenmodell eingegangen werden.

\section{Marx' Kampf mit den Klassen}

Es sollte nicht allzu voreilig von Marx Zweiklassenmodell (Kapital/Arbeit) auf dessen Blindheit gegenüber der Existenz mannigfaltiger Positionen im sozialen Raum geschlossen werden. Besonders deutlich wird Marx' Ringen mit der Einheit der Klasse im dritten Band des Kapitals, das nur knapp über 300 Wörter umfasst. In dem letzten Kapitel seines Monumentalwerkes stellt sich Marx noch einmal die Klassenfrage: „Was bildet eine Klasse?“ (Marx 1968b: 893) Hier erklärt Marx neben den Lohnarbeiter_innen und den Kapitalist_innen den Grundeigentümer zu einer dritten großen Klasse. Nachdem er das Zweiklassenmodell reformuliert und die „Bildner der drei großen gesellschaftlichen Klassen“" nun in drei große Gemeinschaften unterteilt, scheint eine weitere Zersplitterung der Klasseneinheiten nicht mehr auf haltbar:

Indes würden von diesem Standpunkt aus z.B. Arzte und Beamte auch zwei Klassen bilden, denn sie gehören zwei unterschiednen gesellschaftlichen Gruppen an, bei denen die Revenuen der Mitglieder von jeder der beiden aus derselben Quelle fließen. Dasselbe gälte für die unendliche Zersplitterung der Interessen und Stellungen, worin die Teilung der gesellschaftlichen Arbeit die Arbeiter wie die Kapitalisten und Grundeigentümer - letztere z.B. in Weinbergsbesitzer, Äckerbesitzer, Waldbesitzer, Bergwerksbesitzer, Fischereibesitzer - spaltet. (Marx 1968b: 893)

An dieser Stelle bricht das Manuskript ab. Dass Marx das letzte Kapitel seines Monumentalwerkes leichtfertig abgebrochen hat, ist nicht anzunehmen, da doch 
die dialektische Bewegung gerade in der beharrlichen Aufhebung der Gegensätze besteht und das Verharren in der Zersplitterung der Widersprüche ihrer ganz und gar entgegensteht. Noch zu Beginn des Kapitels betont Marx, dass die Zersplitterung der Klassen lediglich einer Übergangsstufe im „Entwicklungsgesetz der kapitalistischen Produktionsweise" entspricht (Marx 1968b: 892). Die Vervielfältigung der Klassen entstehe notwendigerweise durch die zunehmende Scheidung der Produktionsmittel von der Arbeit, die aber gleichzeitig eine zunehmende "Konzentration in große Gruppen“, das heißt die Vereinheitlichung der Klassen, nach sich zieht (vgl. Marx 1968b: 892). Die Zersplitterung der Klassen kann man mit Marx als eine der dialektischen Bewegung inhärente Entwicklungsstufe begreifen, die am Ende in die Revolution als die ereignishafte Überwindung der widersprüchlichen kapitalistischen Gesellschaftsordnung mündet. Am 30. April 1868 schreibt Marx an Engels einen Brief, in dem er den Inhalt der drei Bände des Kapitals skizziert und die Vollendung des Werkes mit dem letzten Kapitel über den Klassenkampf in Aussicht stellt:

Endlich, da jene drei (Arbeitslohn, Grundrente, Profit (Zins)) die Einkommensquellen der drei Klassen von Grundeigentümern, Kapitalisten und Lohnarbeiten - der Klassenkampf als Schluss, worin sich die Bewegung und
Auflösung der ganzen Scheiße auflöst...

(Marx 1968c: 70)

Es ist unschwer zu sehen, dass sich „die Scheiße" dann doch nicht auflöst, sondern sich das Problem der Klassen eher in alle möglichen Richtungen hin verteilt. Im letzten Kapitel fallen die Klassen einer nicht aufzuhaltenden Fragmentierung anheim, wie auch das Kapitel selbst ein Fragment bleibt. Ironischerweise bleibt die dialektische Bewegung nicht nur in der Theorie unvollendet. Die Revolution findet weder in der Theorie noch in der Praxis zu ihrer vollständigen Verwirklichung. Der Kampf ist nicht befriedet.

\section{Bourdieu und die Aufrechterhal- tung der Klassengegensätze}

Aus revolutionär-marxistischer Perspektive stellt die Zersplitterung der Klassengemeinschaften eine bloße Entwicklungsstufe spätkapitalistischer Verhältnisse einer Bewegung hin zur Revolution dar. Hält man an den Glauben an eine Revolution fest, die einen vollständigen Bruch mit den bisherigen Verhältnissen herbeiführen wird, ist es nicht mehr weit zu dem Vorwurf, Bourdieu affirmiere die Interessen des Bürgertums anstatt diese zu kritisieren. Denn Bourdieu stellt weder die Herrschaft des Proletariats in Aussicht noch verweist er auf eine andere (zukünftige) Möglichkeit zur Auflösung der Klassenantagonismen 
in Form einer revolutionären Umwälzung des sozialen Raums.

In dieser Lesart gäbe Bourdieu nur vor, den Habitus der Klassengemeinschaften von einem neutralen Standpunkt aus zu beobachten, nähme dabei jedoch nur einen temporären Ausschnitt des sozialen Raumes wahr, der lediglich eine Entwicklungsstufe spätkapitalistischer Gesellschaften widerspiegele. Aus orthodox-marxistischer Perspektive müsste sich Bourdieu den Vorwurf gefallen lassen, die Fluidität des Sozialen und die Möglichkeit einer revolutionären Umwälzung auf die Tätigkeit vereinzelter Individuen zu beschränken. Deren Handlungsmacht wird auf den Möglichkeitsraum des klassenspezifischen Habitus einzelner Individuen reduziert. Emanzipatorische Prozesse werden auf „Umstellungsstrategien "(Bourdieu 2006a: 227) reduziert, anstatt auf die Veränderbarkeit der Gesellschaftsstruktur zu verweisen. Der emanzipatorische Anspruch der bourdieuschen Theorie wäre dadurch verwirkt, als dass er den Teil der dialektisch-materialistischen Bewegung als Ganzes vorstellt und die Möglichkeit zur Veränderung der sozialen Struktur unberücksichtigt lässt. Um die Veränderung der sozialen Positionen in den Blick zu nehmen, müsste Bourdieu jedoch nicht nur die Ausdifferenzierung der Klassenpositionen im Sozialen erklären, sondern auch, wie sich partikulare Positionen zusammenschließen, um gemeinsam eine neue Aufteilung der sozialen Ordnung zu schaffen.
Man sollte jedoch mit Bourdieu nicht zu streng sein, da doch sogar Marx bei der Zersplitterung des Klassenantagonismus stehenbleibt. Im Nachfolgenden werde ich mit Verweisen auf Laclau/Mouffes postmarxistisches Gemeinschaftsverständnis argumentieren, dass die Zersplitterung und die fehlende Auflösung der Klassenantagonismen keinen Mangel im Sinne eines theoretischen Fehlers darstellt, der hätte vermieden werden können. Vielmehr haben wir es mit der Unmöglichkeit einer letzten Naht des Sozialen zu tun.

\section{Laclau und Mouffes post- marxistisches Verständnis politischer Gemeinschaften}

In ihrem Hauptwerk Hegemonie und radikale Demokratie (1985) entwickeln Laclau und Mouffe in der Auseinandersetzung mit postmarxistisch-radikaldemokratischen Ansatz. In einigen Punkten bleiben sie dem Klassischen Marxismus treu, in vielen anderen entwickeln sie ihn weiter. Sie konstatieren eine

Vielfalt sozialer Verhältnisse [..], von denen Antagonismen und Kämpfe ausgehen können: Wohnen, Konsum, vielfältige Dienstleistungen können ein Terrain für die Kämpfe der Ungleichheiten und für die Forderung nach neuen Rechten bilden. (Laclau/ Mouffe 2015: 196) 
Ebenso wie Bourdieu betonen sie die Streuung der sozialen Positionen in modernen Gesellschaften, die sich nicht ohne Weiteres auf einen Punkt der Vereinheitlichung beziehen lassen. Diese Vervielfältigung von Differenzen verstehen Laclau und Mouffe als Artikulationen im Kontext einer Reorganisation sozialer Verhältnisse in einer Reihe von Veränderungen nach dem zweiten Weltkrieg, die eine neue hegemoniale Formation konsolidierten (vgl. Laclau/Mouffe 2015: 197). In der Analyse dieser neuen hegemonialen Formation in modernen westlichen Gesellschaften scheinen Laclau und Mouffe zunächst einer Marx nahen Diagnose zu folgen: Es kommt immer mehr zu einer „Ausdehnung kapitalistischer Produktionsverhältnisse auf alle sozialen Verhältnisse und ihre Unterordnung unter die Logik der Profitproduktion." (Laclau/Mouffe 2015: 197) Die vorherrschende Konsumptionsweise in der hegemonialen Formation des Fordismus nach dem zweiten Weltkrieg sei durch „den individuellen Erwerb von Waren, die im großen Umfang für die private Konsumption produziert werden, charakterisiert" (Laclau/Mouffe 2015: 97). Es wird so nicht gemeinschaftlich-individuell, sondern kapitalistisch und individualistisch konsumiert, indem die Produkte menschlicher Arbeit von ihren Produzent_innen mehr und mehr abgespalten werden:

Die kapitalistischen Produktionsverhältnisse verwandelten die Gesellschaft seit den vierziger Jahren in gesteigerter Form in einen gewaltigen Markt, in dem unaufhörlich 'Bedürfnisse' geschaffen und immer mehr Produkte menschlicher Arbeit in Waren verwandelt wurden. (Laclau/Mouffe 2015: 97)

Das Spannende an Laclau und Mouffes Ansatz ist jedoch nicht die Kritik an den herrschenden kapitalistischen Produktionsverhältnissen, die sie nicht wesentlich von anderen neomarxistischen und kritischen Gesellschaftsanalysen unterscheiden. Interessant ist, dass sie diese Verhältnisse in besonderen Maßen auch als Chance zur Abschaffung aktuell herrschender Unterdrückungsverhältnisse sehen, die in zukünftigen Gemeinschaftsformen Geschichte sein werden.

Es soll nachfolgend in Anschluss an Laclau/ Mouffe gezeigt werden, dass die Diagnose der „Konsument_innengesellschaft" und die zunehmende Ausweitung kapitalistischer Gesellschaftsverhältnisse nicht zur Resignation und einer daraus resultierenden Tatenlosigkeit führen muss. Im Gegenteil: Durch die Vervielfältigung sozialer Antagonismen im Zuge der Vielfalt unterschiedlicher Konsumptions- und Lebensweisen entsteht die Möglichkeit pluralistisch-demokratischer Ordnungen und emanzipatorischer Bewegungen. Laclau und Mouffe erkennen die Möglichkeit, gleichzeitig die Verstreuung der sozialen Kämpfe, als auch die Etablierung eines 
Antagonismus zu denken, durch den sich unterschiedliche soziale Positionen zusammenschließen, um gemeinsam gegen Formen der Unterdrückung aufzutreten. Anders als bei Marx ist eine solcher Möglichkeitshorizont demokratischer Art und nicht mit einem revolutionären Ereignis und dem darauffolgenden Zusammenschlusses zu einer genähten Gemeinschaft verbunden.

\section{Totalitätskritik und methodi- sche Neuorientierung im Post- fundationalismus}

Laclau/Mouffes Totalitätskritik ist ein entscheidendes Moment ihrer radikaldemokratischen Utopie. Einerseits verstehen sie ihr Projekt als eine Dekonstruktion des Marxismus, durch welches möglich wird, diejenigen Momente in der marxistischen Theoriebildung zu verabschieden, welche demokratischen Ordnungen nicht gerecht werden. Andererseits sehen sie auch schon in der dialektischen Methode bei Marx und vorher bei Hegel, eine Form von Totalitätskritik: „Genau darin besteht Hegels Modernität: für ihn ist Identität niemals positiv und in sich geschlossen, sondern als Übergang, Verhältnis und Differenz konstiutiert.“" (Laclau/Mouffe 2015: 127) Die dialektische Totalitätskritik erkennen sie als solche an, begreifen aber gleichzeitig, dass es heute notwendig ist, diese zu vertiefen. Sie behaupten demnach, dass in der
Dialektik dem Schließungseffekt und dem „notwendigen Charakter eines apriorischen Übergangs mehr Gewicht beigemessen wurde, als dem diskontinuierlichen Moment einer offen Artikulation." (Laclau/Mouffe 2015: 128) Ein potenziell totalitäres SchlieBungsmoment findet sich ihrer Meinung nach auch in der marxistischen Theoriebildung: Laclau und Mouffe beschreiben die Möglichkeit einer „autoritären Wendung“, die ,in mancher Hinsicht seit den Anfängen der marxistischen Orthodoxie" gegeben war (Laclau/Mouffe 2015: 88). Das Aufkommen dieser Möglichkeit ergab sich präzise in jenem Augenblick, „als ein eingegrenzter Akteur - die Arbeiterklasse - auf den Status einer 'universellen Klasse' gehoben wurde.“ (Laclau/Mouffe 2015: 88) In der kommunistischen Utopie verliert die Arbeiterklasse in ihrer Verwirklichung nach der Revolution ihren partikularen Charakter und wird zu einem Gesellschaftskörper, der kein Außen kennt. In ihr soll die Konsumption, die produktiv ist, in den Diensten aller gestellt werden. So persistiert bei Marx eine Art messianisches Moment als die Hoffnung auf eine vollständige Versöhnung aller Differenzen in einer letzten identitätslogischen Einheit. Laclau und Mouffes Ansatz hingegen formuliert eine Totalitätskritik, die sich als postfundationalistisch versteht. Postfundationalistisch ist diese Perspektive, weil sie das fundamentalistische Moment einer Vorstellung eines letzten Grundes des Sozialen verabschiedet. Postfundationalistisch ist sie, weil sie deswegen nicht 
behauptet, dass es überhaupt keine Fundamente mehr gebe. Es bleibt notwendig ist, neue Gründe zu verhandeln und aufgrund von neuen Auseinandersetzungen neue Entscheidungen zu treffen.

\section{Neue Gemeinschaften, andere Konsumptionsweisen, andere Lebensweisen}

Im Sinne einer postfundationalistischen Kritik bedeutet es, dass die Ablehnung eines einzigen privilegierten Akteurs nicht bedeuten muss, dass es keine Akteure geben kann, die sich auf Grundlage einer Zersplitterung zusammenschließen, um ein emanzipatorisches Projekt zu verfolgen. Im Folgenden soll geschildert werden, wie ein solcher Zusammenschluss jenseits genähter Totalitäten mit Verweis auf Laclau/ Mouffes Gemeinschaftsverständnis aussehen könnte. Behaupten wir nun, es gäbe ein Unterdrückungsverhältnis im sozialen Raum. Eine, nennen wir sie „Gemeinschaft der Unterdrückten“, konsumiert, um zu leben, und das Leben zu erweitern, zu bereichern und zu befördern. Die Gemeinschaft der Unterdrückenden hingegen treibt den Lebensprozess als eine sinnentleerte Form voran, häuft Kapital an und beutet das Leben der anderen aus. Wer könnte diese Gemeinschaft der Unterdrückten heute sein? „Arbeiter_innen“, „Arbeitslose“, „Angestellte“, „Flüchtlinge“, „Menschen mit Behinderung“, „Konsument_innen“,
„Einkommensschwache“, „Alleinerzieher innen“, „Frauen“, „Transgenderpersonen“, "Jugendliche“, oder auch „die Tiere“, „die Umwelt“? (Scheibenpflug 2016: 83f.). Obwohl Laclau und Mouffe nicht explizit von unterschiedlichen Konsumptionsweisen sprechen, könnte man die Medien, die die Menschen konsumieren, den Sport, den sie treiben, oder das, was die Personen essen und kaufen etc. als konstitutiv für die Artikulation der sozialen Positionen im diskursiven Feld betrachten. Die Konsumptionsweisen sind Teil der diskursiven Struktur, obgleich es kein einziges bestimmendes Prinzip für die Herausbildung sozialer Positionen gibt. So lässt sich mit Laclau und Mouffe argumentieren, dass die Vielfalt der sozialen Positionen und die Unmöglichkeit, die sozialen Akteure eindeutig abzugrenzen, eine eindeutige Bestimmung eines zu emanzipierenden Subjekts unzulässig machen. Im Zuge der Streuung und Unabschließbarkeit der sozialen Position stellt sich aber immer noch die drängende Frage: „Wie kann ein emanzipatives politisches Projekt verfolgt werden, wenn Ungewissheit über die zu emanzipierenden Identitäten (z.B. das Proletariat, die Frauen, ethnische Minderheiten etc.) besteht?" (Stäheli 2009: 255) Wenn kein Subjekt abgegrenzt werden kann, das die Universalität der Gemeinschaft differenzieller Positionen verwirklichen könnte, wie kann es dann zu einem Zusammenschluss zwischen Feminist_innen, Tierrechtler_innen, Gewerkschaftler_innen 
etc. kommen? Die Antwort kann sich nicht in dem Festhalten an ein einziges abgegrenztes revolutionäres Subjekt (wie z.B. den_die Arbeiter_in oder auch den_die Geflüchtete_n) erschöpfen, weil damit stets die Gefahr einer Naht neuer Totalitäten verbunden ist.
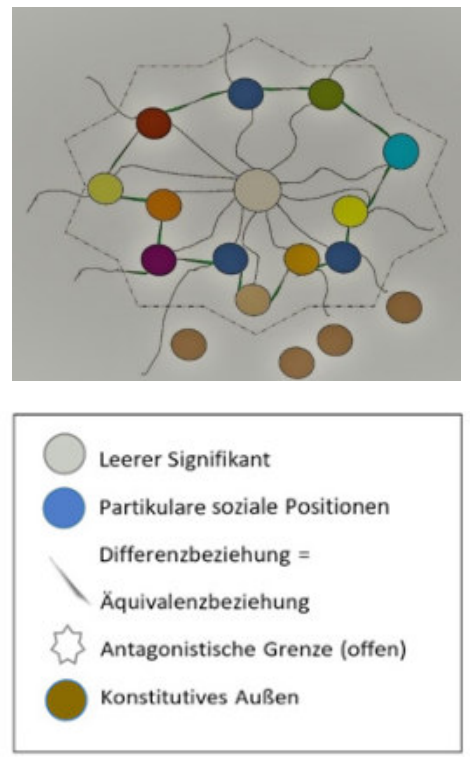

Abb. 2 Diskursive Formation (temporär und flottierend) (vgl.Scheibenpflug 2016: 87).

Laclau und Mouffe gehen einen anderen Weg: Sie konzipieren das Soziale als diskursive Struktur, die nicht als geschlossener Raum gedacht werden kann, sondern als offenes Feld, das stetiger Veränderung unterworfen ist und in das Symbolisches und Materielles verwoben sind (vgl. Laclau/
Mouffe 2015: 143). Die „[...] sprachlichen und die nicht-sprachlichen Elemente werden nicht bloß nebeneinandergestellt, sondern konstituieren ein differentielles und strukturiertes System von Positionen, das heißt einen Diskurs." (Laclau/ Mouffe 2015: 143) Theoretisch-symbolische und materielle Artikulationen sind demnach gleichursprünglich, weshalb mit der Theorie-Praxis-Opposition gebrochen werden muss. Für sie ist Theoriebildung ein praktischer, körperlich-materieller Vollzug, genauso wie der Besuch eines Theaterstückes, das Lesen eines Textes oder die Teilnahme an einer Demonstration.

Die diskursive Struktur begreifen Laclau/ Mouffe als ein Feld flottierender Signifikanten, in der es zur Bildung von Knotenpunkten kommt, die niemals vollständig voneinander abgrenzbar sind und die nie in einem vollständig geschlossenen sozialen Raum fixiert sind (vgl. Laclau/ Mouffe 2015: 177). Das Feld flottierender Signifikanten ist beweglich, es bilden sich ständig neue Formationen und neue Verbindungen. Knotenpunkte in dem Feld flottierenden Signifikanten entstehen, indem sie sich um einen leeren Signifikanten gruppieren. In unserem Beispiel ist es ein partikularer Signifikant "Gegen den Sozialabbau der Regierung " für eine Demonstration, dem es gelingt, eine hegemoniale Funktion zu übernehmen und so die Gleichheit der partikularen Forderungen von Feminist_innen, Menschen 
mit Behinderung, Alleinverdiener_innen, Arbeitslosen etc. zu artikulieren. Der Signifikant ist leer, weil er die Grenzen dessen, was er repräsentieren soll, nicht bezeichnen kann. Seine Artikulation ist deshalb hegemonial und temporär, weil er die Funktion nicht erfüllen kann, das Ganze der Kette, ihre Universalität, zu repräsentieren. Hier unterscheidet sich die postfundationalistische Konzeption wesentlich von der fundamentalistischen Totalitätsvorstellung: Die hegemoniale Artikulation ist immer begrenzt, demnach partikular und kann niemals das Universelle der Gemeinschaft repräsentieren. Sie konstituiert sich stets in Abgrenzung zu einem Außen, das immer nur temporär konstitutiv für das Innen der Gemeinschaft ist und sich von diesem nie vollständig separieren lässt.

\section{Fazit}

Im Zuge einer Gegenüberstellung der gemeinschaftsbildenden Funktion des Konsumierens bei Marx und Bourdieu stellte sich heraus, dass die Erweiterung des Marxschen Konsum- und Klassenbegriffes durch Bourdieu mit einer Zersplitterung des Klassenantagonismus einhergeht. Auch Laclau/Mouffe vertreten die These, dass es in modernen demokratischen Gesellschaften zu einer Vervielfältigung sozialer Antagonismen kommt, denen ein Zweiklassenmodell nicht gerecht wird. In ihrem radikaldemokratischen Projekt verwerfen sie nicht nur die privilegierte Rolle der Arbeiterklasse als revolutionären Akteur, sondern auch die privilegierte Rolle des Konsumierens als Indikator für die Konstitution politischer Gemeinschaften. Allen voran verweisen sie auf die Notwendigkeit neue Signifikanten zu schaffen, die den Gegebenheiten und Möglichkeitshorizonten unserer Zeit gerecht werden. Die Frage nach Konsumption und Gemeinschaft ist verknüpft mit einer alten philosophischen Fragestellung, die immer wieder neu gestellt werden muss und die immer schon eine politische war: Wie wollen wir leben?

\section{ZUR AUTORIN}

Valerie Scheibenpflug ist 27 Jahre alt und schreibt an ihrem Dissertationsvorhaben mit dem Arbeitstitel „Gemeinschaft. absolut-sein. Unmöglich-sein“. In Wien hat sie Philosophie, Psychologie und Germanistik studiert und als Lehrerin gearbeitet. Während ihres Studiums verbrachte sie Forschungsaufenthalte in Nottingham und Heidelberg und war in verschiedenen Studierendeninitiativen tätig. Seit 2017 ist sie Mitarbeiterin im Redaktionsteam von engagée - Journal für politisch-philosophische Einmischungen (www.engagee.org). 
Der Beitrag wurde von Hendrik Erz, Markus Kohlmeier und Veronika Riedl gereviewed. Hendrik Erz und Veronika Riedl haben den Überarbeitungsprozess des Manuskriptes redaktionell betreut. Tanja Strukelj, Tamara Schwertel und Andreas Schulz haben den Artikel lektoriert.

\section{LITERATUR}

Bourdieu, Pierre (2016a): Sozialer Raum und »Klassen«: Zwei Vorlesungen. Frankfurt am Main: Suhrkamp.

Bourdieu, Pierre (2016b): Die feinen Unterschiede. Kritik der gesellschaftlichen Urteilskraft. Frankfurt am Main: Suhrkamp.

Laclau, Ernesto/Mouffe, Chantal (2015): Hegemonie und radikale Demokratie. Zur Dekonstruktion des Marxismus. Wien: Passagen.

Marx, Karl (1968a): Das Kapital. Bd. I. Marx/Engels-Werke. Band 23. Berlin/DDR: Dietz.

Marx, Karl (1968b): Das Kapital. Bd. III. Marx/Engels-Werke. Band 25. Berlin/DDR: Dietz.

Marx, Karl (1968c): Marx/Engels-Werke. Band 32. Berlin/ DDR: Dietz.

Marx, Karl (1977): Das Elend der Philosophie. Marx/ Engels-Werke. Band 4. Berlin/DDR: Dietz.

Scheibenpflug, Valerie (2016): Einheit - Differenz - Werden. Subjektverständnis bei Habermas, Deleuze/Guattari und Laclau/Mouffe. Unveröffentlichte Diplomarbeit. Universität Wien. Online verfügbar unter http://othes.univie. ac.at/42828/ (20.07.2018).

Stäheli, Urs (2009): Die politische Theorie der Hegemonie. Ernesto Laclau und Chantal Mouffe. In: Brodocz, André/ Schaal, Gary (Hrsg.): Politische Theorien der Gegenwart 2. Opladen: Barbara Budrich, S. 143-166. 


\section{$M$ MAX \\ M 1 The A At of Data Analyss}

\section{Professionelle Software für}

\section{Qualitative \& Mixed Methods Forschung}

Erfolgreich studieren mit MAXQDA: Selbstgeführte Interviews einfach und effizient transkribieren und auswerten. Flexible Analyse von Bildern, Webseiten, Tweets, Umfrageergebnissen, u.v.m.. Schnelle Erstellung von Literaturreviews mit cleverer Verschlagwortung und Themensuche. In der Variante MAXQDA Analytics Pro mit Stats-Modul für statistische Auswertungen.

Daten erheben - organisieren - analysieren - visualisieren - präsentieren

Unschlagbare Preise für Studierende und Promovierende:
$45 €$

MAXQDA Standard Laufzeit: 6 Monate
$89 €$

MAXQDA

Standard

\section{$99 €$}

MAXQDA

Analytics Pro

Laufzeit: 1 Jahr
Laufzeit: 2 Jahre
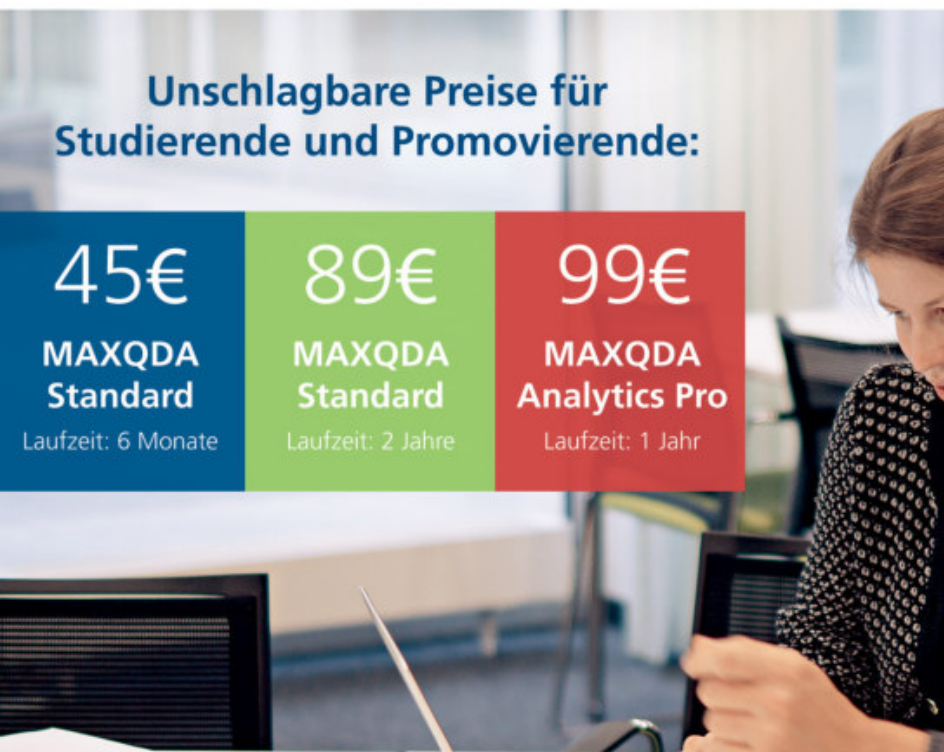
$$
\text { REDAKTION }
$$
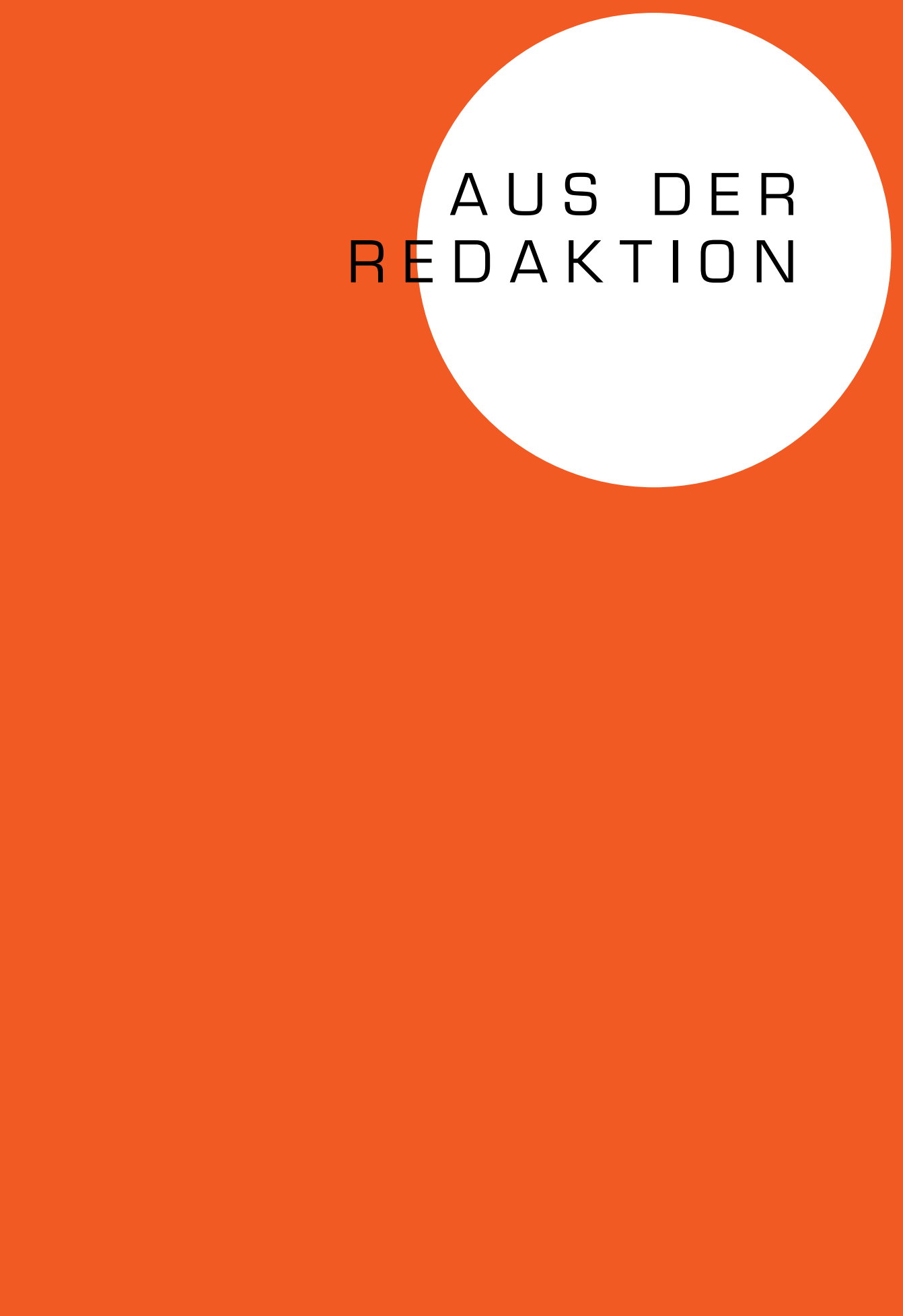


\section{Soziologiemagazin online}

\section{MACH MIT!}

\section{... im Redaktionsteam}

Eine eMail genügt! Wir suchen stets neue Gesichter mit frischen Ideen. Aktuell brauchen wir Unterstützung insbesondere für das Lektorat, den Satz, die Autorenbetreuung sowie für die Durchführung von Interviews für unseren YouTubeChannel.

Wenn ihr nicht zum ständigen Redaktionsteam gehören wollt, gibt es die Möglichkeit, uns im Rahmen des Kuratoriums (bzw. Freundeskreises) mit Rat und Tat zur Seite zu stehen.

\section{... als Autor_in in unserem Magazin}

Schickt uns zu unserem aktuellen Call4Papers eure wissenschatlichen Artikel. Außerdem nehmen wir in unseren Serviceteil „Perspektiven” gerne auch Rezensionen, Tagungsberichte, Interviews oder andere soziologische Inputs mit auf.

\section{... als Blogger_in}

Schickt uns eure Ideen für Rezensionen aktueller soziologischer Bücher, eigene soziologische Blog-Beiträge oder Interview-Vorschläge an: beiträge@soziologiemagazin.de

Meldet euch bei uns oder leitet einen Hinweis auf uns in eurem soziologisch interessierten Umfeld weiter. Wir freuen uns!
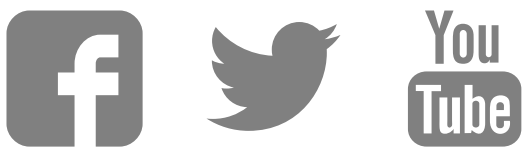


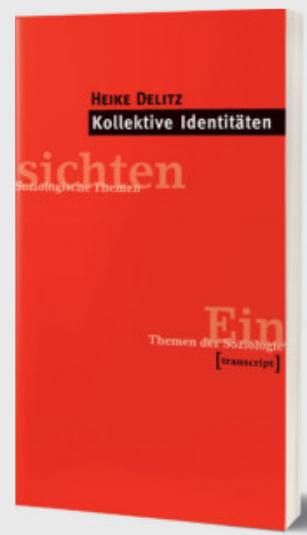

\section{MINUTEN} MATERIALITÄT

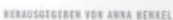

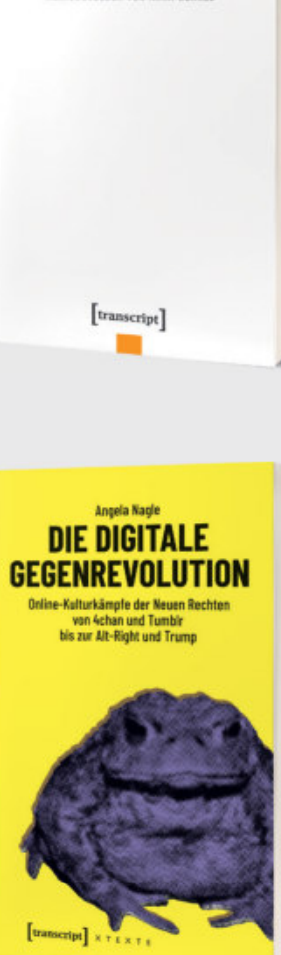

Heike Delitz

\section{Kollektive Identitäten}

August 2018, 160 Seiten, kart.,

$14,99 €$, ISBN 978-3-8376-3724-3,

E-Book: $12,99 €$

Kollektive Identitäten sind ein hochaktuelles gesellschaftliches Thema - und ein grundlegendes Konzept der Sozial- und Kulturwissenschaften. Diese Einführung gibt einen profunden und systematischen Überblick und eignet sich vor allem für Studium und Lehre.

\section{Anna Henkel (Hg.)}

\section{Minuten Soziologie: Materialität}

Juni 2018, 122 Seiten, kart., 15,99 €, ISBN 978-3-8376-4073-1,

E-Book: 13,99 €

In jeweils "10 Minuten " schärfen die einzelnen Beiträge des Bandes den Blick auf das Soziale an Materialität in je einer ihrer Facetten. Die Reihe präsentiert ein innovatives Konzept, das einen leichten Zugang zu Themen der Soziologie ermöglicht. Nicht nur für Studium und Lehre geeignet!

\section{Angela Nagle}

Die digitale Gegenrevolution

Online-Kulturkämpfe der Neuen Rechten

von 4chan und Tumblr bis zur Alt-Right und Trump

August 2018, 148 Seiten,

kart., 19,99 €, ISBN 978-3-8376-4397-8,

E-Book/EPUB: $17,99 €$

In inrem Bestseller beschreibt Angela Nagle die neuen OnlineKulturkämpfe und ihre Protagonisten, von den Neokonservativen, Rechtsextremen und wütenden Nerds der Neuen Rechten zum demonstrativen "Gutmenschentum» der Linken.

"Das Buch trifft einen Nerv."

Nana Brink, Deutschlandfunk Kultur,

13.01.2018 zur englischsprachigen Ausgabe 


\section{Literaturübersicht zur}

\section{Konsumsoziologie}

\section{Buchempfehlungen der Redaktion}

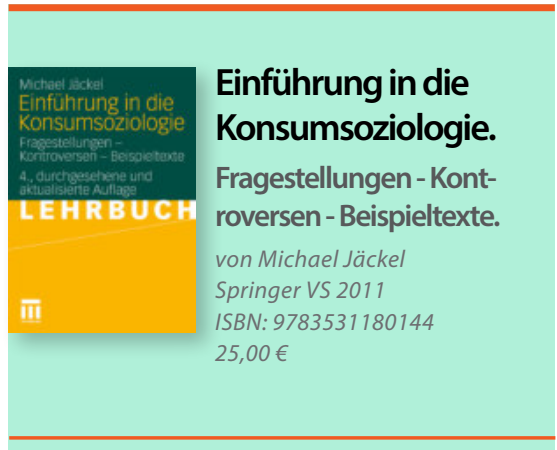

Das Buch, das sich als übersichtliche Einführung in die Konsumsoziologie eignet, gibt Einblicke in die historische Entwicklung der Konsumgesellschaft und beschäftigt sich mit gegenwärtigen Phänomenen. Michael Jäckel geht insbesondere auf die Frage der Bedürfnisse, auf Werbeformen und Orte des Konsums sowie aufden Einfluss des sozialen Kontexts auf Konsumgewohnheiten ein. Die ausgewählten Beispieltexte komplettieren die Analysen und vereinfachen die Suche nach vertiefender Lektüre zu ausgewählten Fragestellungen.

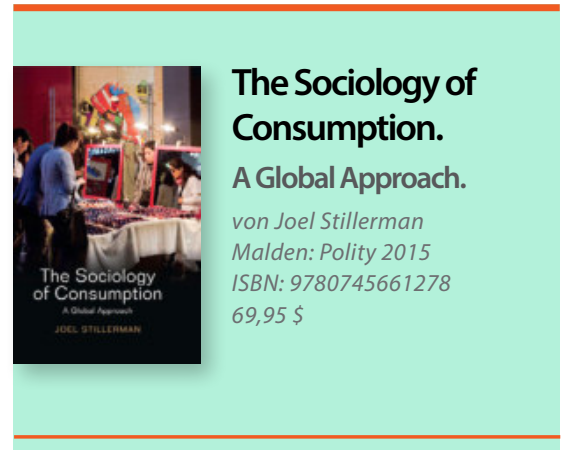

Das Buch untersucht den Einfluss von Globalisierung auf den Wandel des Konsums und soziale Ungleichheiten in Verbindung mit Konsumpraktiken. Joel Stillerman macht auf die Ausdehnung des Konsums in Ländern außerhalb des Globalen Nordens und die Variation von Konsummustern durch transnationale Einflüsse aufmerksam. Der Autor geht außerdem auf die Frage ein, wie Konsum Ungleichheit widerspiegelt und wie Individuen versuchen, ihren sozialen Status durch Konsum zu beeinflussen. Abschließend trägt das Buch zur Debatte um Consumer Citizenship bei. 


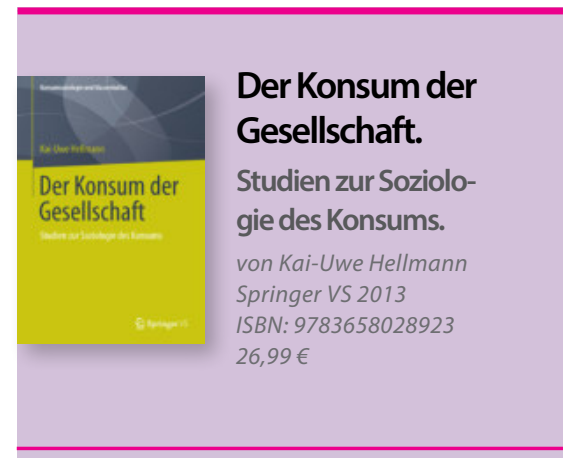

Die Aufsatzsammlung des Konsumsoziologen, der unter anderem auch So- ziologie der Marke (2003) veröffentlicht hat, beschäftigt sich mit aktuellen Fragestellungen, die das Thema Konsum in modernen Gesellschaften aufwirft. Die Essays schneiden diverse Themengebiete an, wie den Konsum von Nahrung, die Rolle der Kund_innen und deren Wandel, sowie Transformationen der Konsumgewohnheiten bedingt durch technologischen und wirtschaftlichen Wandel. Der Autor liefert dabei einen guten Überblick über die Konsumsoziologie, ihre Grenzen und zukünftige Forschungsfelder.

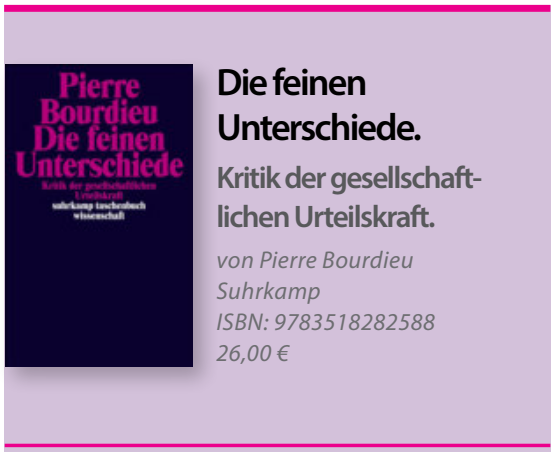

Das über die Grenzen der Disziplin hinaus bekannte Buch macht auf die soziale Dimension von Konsumpräferenzen und Freizeitinteressen aufmerksam und rahmt Konsum als Distinktionsmechanismus. Konsumpraktiken spiegeln somit das Klassensystem der Gesellschaft wieder. Basierend auf umfangreichen empirischen Studien macht Bourdieu deutlich, dass Konsum mehr ist als die Befriedigung primärer Bedürfnisse. Trotz der vorgebrachten Kritik an den theoretischen Grundannahmen hatte das Buch deutlichen Einfluss auf konsumsoziologische Fragestellungen sowie auf über Konsumstile definierte Lebensstilkonzepte. 


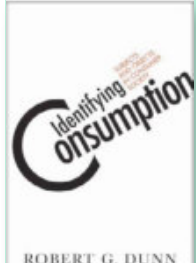

ROBERT G. DUNN

\section{Identifying}

Consumption.

Subjects and Objects in Consumer Society.

von Robert G. Dunn

Temple University Press 2008

ISBN: 9781592138708

$23,95 \$$

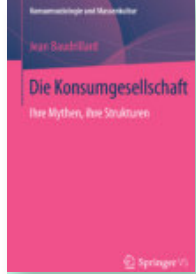

Die Konsumgesellschaft.

Ihre Mythen, ihre

Strukturen.

von Jean Baudrillard

Springer VS 2015

ISBN: 9783658005405

$24,99 €$
Das Buch fasst soziologischen Perspektiven auf Konsumpraktiken zusammen und liefert neue theoretische Konzepte um das Verhalten von Konsument_ innen zu analysieren. Robert Dunn stellt einen strukturalen und prozessualen Rahmen vor, der es ermöglicht Strukturbedingungen und die individuelle Subjektivität des Konsums zu verbinden. Der Autor plädiert für eine konsumsoziologische Analyse, die sowohl Kommodifizierungs- und Kommerzialisierungsprozesse als auch Lebensstil, Status und Identität der Akteure einbezieht.
Das erst kürzlich aufDeutsch erschienene Buch des französischen Philosophen und Soziologen baut auf strukturalistischen und semiotischen Ansätzen auf. Konsum ist in dieser Lesart ein System von Zeichen und Bedeutungen - ein Mythos der alle gesellschaftlichen Bereiche durchdringt. Damit durchbricht Baudrillard die vereinfachende Gegenüberstellung von Produktion und Konsumption. Baudrillard zeigt auf, wie der Konsum die sozialen Beziehungen in westlichen Gesellschaften strukturiert und liefert eine durchaus heute noch lesenswerte Zeitdiagnose. 


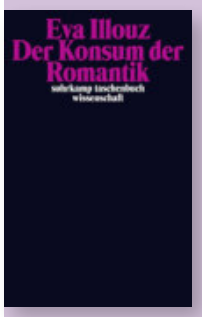

\section{Der Konsum der Romantik.}

Liebe und die kulturellen Widersprüche des

Kapitalismus.

von Eva Illouz

Suhrkamp 2007

ISBN 9783518294581

$17,00 €$
Eva Illouz beschäftigt sich mit der Rolle von Emotionen im Kapitalismus und stellt die These auf, dass Romantik in individualisierten spätkapitalistischen Gesellschaften zunehmend kommodifiziert und rationalisiert wird. Dass Romantik zu einem wichtigen Bestandteil des kapitalistischen Ethos geworden ist und dass der ,ideale romantische Moment durch Konsum ermöglicht wird, wird jedoch durch die emotionale Intensität verschleiert. Die Studie, basierend auf diversen historischen und aktuellen Quellen, liefert überzeugende soziologische Konzepte um romantische Praktiken aus konsumsoziologischer Perspektive $\mathrm{zu}$ beleuchten.

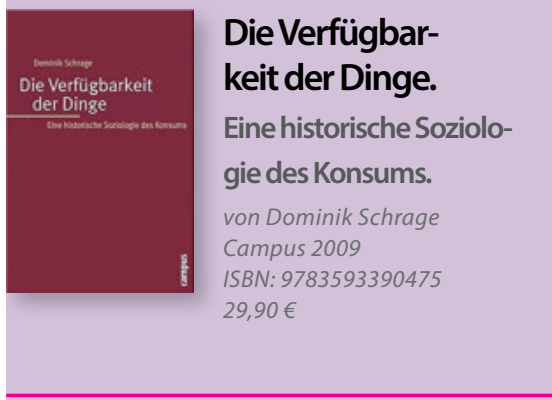

Der Autor zeichnet die Genealogie des Konsums von der Frühen Neuzeit bis zum modernen Massenkonsum nach. Nach einer detaillierten Analyse klassischer theoretische Ansätze der Konsumsoziologie nähert Schrage sich dem Thema mit begriffsgeschichtlichen Überlegungen an. Ausgangspunkt seiner Überlegungen zu Wandel und Bedeutung des Konsums ist die These, dass Konsum, beziehungsweise die „Verfügbarkeit der Dinge“, Medium moderner Vergesellschaftungsprozesse ist. Das historisch-genealogische Werk bereichert die soziologische Diskussion um neue Thesen bezüglich der Beziehung von Dingwelt und Gesellschaft. 


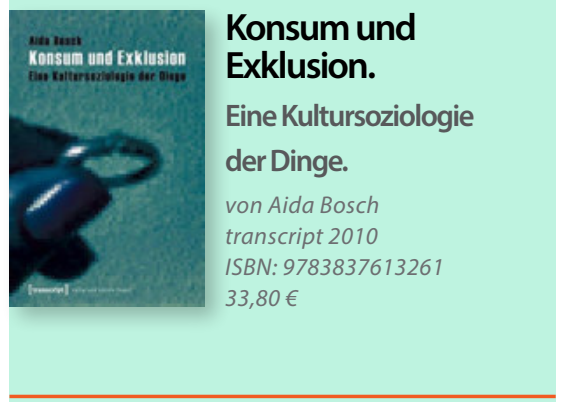

Die Autorin analysiert symbolische Formen der Inklusion und Exklusion und entwickelt eine Soziologie der Dingwelt. In spätkapitalistischen Gesellschaften kommt dem Konsum eine wichtige Bedeutung für die gesellschaftliche Integration und die Identitätskonstruktion zu. Soziale Inklusion ist an den Besitz von bestimmten Konsumgütern gebunden und wird durch sie symbolisiert. Nach Religion und Arbeit tritt nun der Konsum als Vergesellschaftungsmodus ins Zentrum. Dies lässt umgekehrt die Frage nach der Exklusion gesellschaftlicher Milieus in sozialen Randlagen aufkommen. Diesekulturwissenschaftliche Perspektive erlaubt es, den Zusammenhang zwischen Konsumsoziologie und Ungleichheitsforschung neu zu denken.

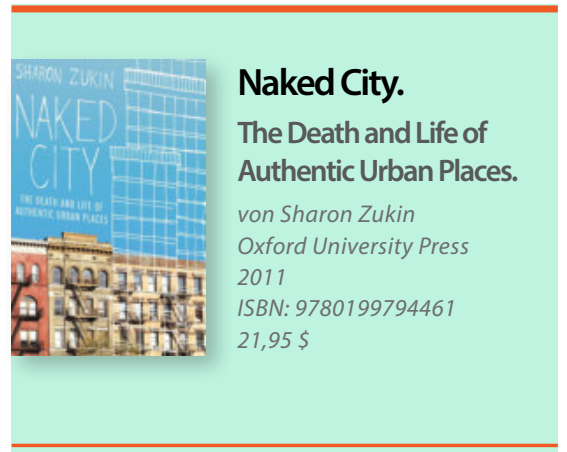

Das Buch untersucht die Verbindung zwischen authentischem Konsum und der Transformation urbaner Viertel am Beispiel von New York. Die Suche nach Authentizität unter städtischen Konsument_innen lässt neue Konsumpraktiken entstehen, die die Stadtentwicklung beeinflussen. Das Auftauchen, authentischer' hochpreisiger Cafés, Märkte und Restaurants trägt zur Bildung lokaler Identitäten bei, beeinflusst aber auch Wohnkosten und stellt somit einen Exklusionsfaktor für die ärmeren Bewohner_innen der Viertel dar. Sharon Zukin analysiert Gentrifizierungsprozesse indem sie nicht nur wirtschaftliche Faktoren, sondern auch die kulturelle Macht der Konsument_innen mit einbezieht.

Die Literaturauswahl wurde von Veronika Riedl zusammengestellt. Tatiana Huppertz und Andreas Schulz haben den Beitrag lektoriert. 


\section{Tagungen und Termine}

(1) Empowerment in Zeiten von Fluchtmigration

Eine internationale Konferenz am 15. und 16. November 2018 an der BTU CottbusSenftenberg.

2 Postpatriarchale Gesellschaft? Struktur und Dynamik II Ein Workshop am 30. November 2018 an der Universität Paderborn.

3 Angst und Regression: Gesellschafts- und Kulturwissenschaftliche Perspektiven

Eine Interdisziplinäre Tagung am 6. und 7. Dezember 2018 am Institut für Soziologie der Universität Gießen.

(4) Protestkommunikation: Umstrittene Legitimität politischer Öffentlichkeit in digitalen und urbanen Räumen

Ein Workshop am 14. und 15. Dezember 2018 an der Universität Salzburg.

5 Demographic Conference of "Young Demographers" Actual Demographic Research of Young Demographers (not only) in Europe

Am 7. und 8. Februar 2019 in Prag.

6 Care.Macht.Mehr. Zwischen Dystopien und Utopien: Nachdenken über die Zukunft des Sorgens und der Sorgearbeit

Ein Workshop am 14. und 15. Februar an der Johannes Kepler Universität Linz.

(7) 40 Jahre „Die feinen Unterschiede" - Zur Aktualität von Pierre Bourdieus Gesellschaftstheorie in der kultursoziologischen Ungleichheitsforschung Eine Tagung vom 13. bis 15. März 2019 an der Universität für Musik und darstellende Kunst Wien.

8 Turbulent Times in Europe: Instability, Insecurity and Inequality

Die vierte European Social Survey Konferenz vom 15. bis 17. April an der Universität Mannheim. 


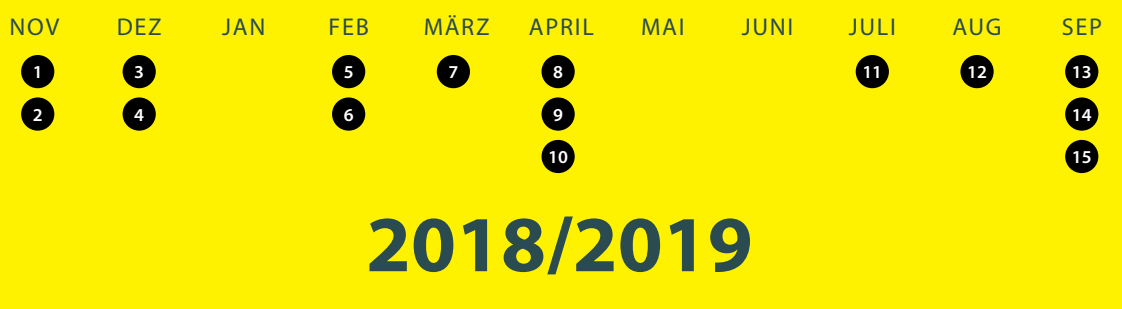

9 Fragmentations and Solidarities

Die 37. internationale Labour Process Conference vom 24. bis 26. April 2019 an der Universität Wien.

(10) Datenvielfalt: Potentiale und Herausforderungen

Tagung des Netzwerks Qualitative Methoden am 25. und 26. April an der Universität Salzburg.

(11) Ethnographie der Situation Erkundungen sinnhaft eingrenzbarer Feldgegebenheiten

Die 7. Fuldaer Feldarbeitstage am 5. und 6. Juli 2019.

12 Europe and Beyond: Boundaries, Barriers and Belonging

Die 14. European Sociological Association Konferenz vom 20. bis 23. August in Manchester.

13 Great Transformation: Die Zukunft moderner Gesellschaften

Abschlusskonferenz der DFG-Kollegforscher_innengruppe „Postwachstumsgesellschaften“ und 2. Regionalkonferenz der DGS an der Friedrich-Schiller-Universität Jena vom 23. bis 27. September 2019

(14 Alles im Wandel? Dynamiken und Kontinuitäten gegenwärtiger Gesellschaften Der ÖGS-Kongress vom 26. bis 28. September 2019 in Salzburg.

\section{(15) Comparing Colonialism: Beyond Modern Exceptionalism}

Eine interdisziplinäre Konferenz vom 26. bis 28. September an der Universität Basel.

Die Terminübersicht wurde von Andreas Schulz recherchiert und von Tatiana Huppertz lektoriert. 


\section{Redaktionsteam}

Andreas Schulz (M.A.), studiert Kultur- und Sozialanthropologie sowie Publizistik- und Kommunikationswissenschaft an der Universität Wien. Aufgaben: Vereinsvorstand, Heftund Lektoratskoordination, Autor_innenbetreuung und Review.

Claas Pollmanns (M.A.), promoviert in Chemnitz. Aufgaben: Vereinsvorstand, Autor_ innenbetreuung und Review.

Clément Dréano (M.A.), promoviert in Turin und Mailand. Aufgaben: Autor_innenbetreuung und Review.

Eva-Maria Bub (Dr. des.), hat an der Universität Frankfurt/Main promoviert und ist derzeit wissenschaftliche Mitarbeiterin an der TU Darmstadt. Aufgaben: Betreuung wissenschaftlicher Beirat, Social Media, Lektorat, Blogkoordination und Review.

104 Franziska Deutschmann (M.A.), Studium der Soziologie und Musikwissenschaften in Frankfurt/Main. Aufgaben: Autor_innenbetreuung, Lektorat und Review.

Hendrik Erz (M.A.), hat Geschichte, Politikwissenschaften und Sozialwissenschaften in Bonn studiert und ist derzeit wissenschaftlicher Mitarbeiter an der Universität Bonn. Aufgaben: Öffentlichkeitsarbeit, Autor_innenbetreuung und Review.

Laura Porak (B.A.), studiert Soziologie an der Universität Wien und Volkswirtschaftslehre an der Wirtschaftsuniversität Wien. Aufgaben: Review.

Markus Kohlmeier (M.A.), promoviert an der Universität Bamberg. Aufgaben: Anzeigen, Autor_innenbetreuung und Review.

Sam Schneider (B.A.), studiert Soziologie in Frankfurt/Main. Aufgaben: Lektorat.

Sarah Kaschuba (M.A.), studierte Militärsoziologie und Militärgeschichte an der Universität Potsdam und der University of Mississippi. Aufgaben: Finanzvorstand.

Tamara Schwertel (B.A.), studiert Soziologie mit Schwerpunkt empirische Methoden der Sozialforschung sowie Philosophie in Frankfurt/Main. Aufgaben: Heftkoordination, Autor_innenbetreuung, Lektorat und Review.

Tanja Strukelj (B.A.), studiert Soziologie in Frankfurt/Main. Aufgaben: Lektorat.

Tatiana Huppertz (M.A.), arbeitet als Semantic Expert bei der cognesys GmbH in Aachen. Aufgaben: Lektorat.

Veronika Riedl (B.A.), studiert Soziologie an der Universität Innsbruck und der Université du Québec à Montréal. Aufgaben: Review, Autor_innenbetreuung, Layout, Satz und Bildredaktion.

Wibke Henriette Liebhart (M.A.), promoviert im Forschungsprojekt "Politics of Wonder” am Soziologieinstitut der Universität Freiburg. Aufgaben: Autor_innenbetreuung, Lektorat und Review. 


\section{Externe Reviewer_innen:}

Felix Fink (M.A.), hat Soziologie in Freiburg/Breisgau studiert. Seine Schwerpunkte umfassen die Themen Affekte und Medien und Poststrukturalistische Theorie.

Maik Krüger (M.A.), ist für wissenschaftliche Mitarbeit an der LMU München angestellt und promoviert dort.

Philipp Reimann (M.A.), ist am Lehrstuhl für Systemanalyse und Vergleichende Politikwissenschaft - Bereich Methoden der Politikwissenschaft an der Martin-Luther-Universität Halle-Wittenberg beschäftigt. Seine Schwerpunkte umfassen die Themen der politischen Soziologie, Theorien abweichenden Verhaltens sowie Konsequenzen des Straf- und Justizvollzugs.

Tristan Dohnt (M.A.), ist wissenschaftlicher Mitarbeiter im Fachbereich Soziologie und Gender Studies an der LMU München.

\section{DANKSAGUNG}

Das Soziologiemagazin wird - samt dem dazugehörigen Verein - ausschließlich von ehrenamtlich arbeitenden Menschen getragen: Studierende und Absolvent_innen der Soziologie und/oder verwandter Fächer, aber auch Promovierende sowie den wissenschaftlichen Mitarbeiter_innen und Professor_innen, die sich bei uns als wissenschaftliche Beiräte engagieren. An all diejenigen möchten wir auch diesmal ein herzliches und großes Dankeschön aussprechen. Danke für Eure und Ihre Energie, für die investierte Zeit und Mühe, für Diskussionen und Absprachen sowohl in der Redaktion als auch mit den Autor_innen. Ein solches Engagement ist nicht selbstverständlich und soll deshalb an dieser Stelle dezidiert bedacht, genannt und gewürdigt werden! Des Weiteren durften wir uns auch diesmal mit zahlreichen und diversen Beiträgen auseinandersetzen; vielen Dank an die dazugehörigen Autor_innen, die Lust, Zeit und vielleicht in manchen Fällen auch Mut gefunden haben, ihre Artikel einzusenden und sich dem Review-Verfahren zu stellen. Ohne solche Einsendungen und Rückmeldungen wäre unsere Arbeit frustrierend oder sogar schlicht unmöglich. Außerdem bedanken wir uns beim Verlag Barbara Budrich für die produktive und zuverlässige Zusammenarbeit. So, und das letzte große Dankeschön geht an die Leser_ innen unserer Magazine und des Blogs und an die Menschen, die uns auf Facebook, Twitter und YouTube folgen. Aufgrund Eurer starken Unterstützung macht es uns wiederum großen Spaß, das Magazin - mit allem, was dazu gehört - auf die Beine zu stellen und damit auch weiterhin eine Publikationsplattform für Studierende und Promovierende der Sozialwissenschaften zu bieten. 


\section{DE DE GRUYTER}

$\bar{G}$

\section{ZEITSCHRIFT FÜR SOZIOLOGIE}

\section{Abo-Preis für Studierende: ab nur 45,-€/Jahr}

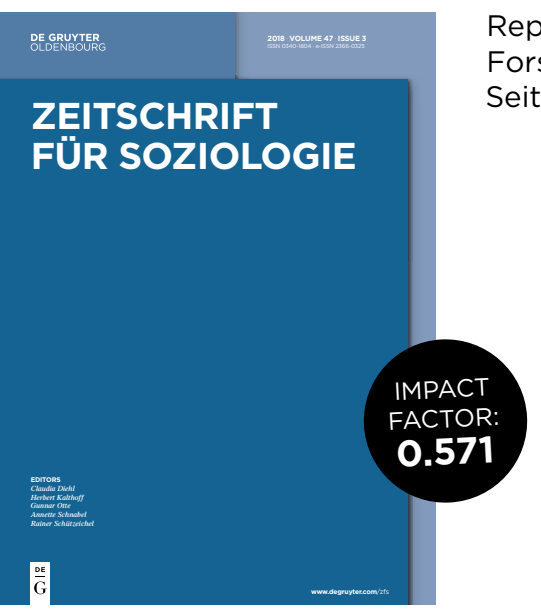

Repräsentatives Organ für empirische

Forschung und soziologische Theoriebildung.

Seit 1971.

- Eine der führenden deutschen Soziologie-Zeitschriften, deren Beiträge gerade für das Studium in vielen soziologischen Disziplinen relevant sind

- Erscheint sechsmal jährlich mit je vier Forschungsartikeln

- Überregionales, pluralistisch besetztes und rotierendes HerausgeberInnengremium

- Beiträge in deutscher und englischer Sprache

- Qualifiziertes Peer-Review-Verfahren durch sieben ExpertInnen des Fachs 


\section{Impressum}

HERAUSGEBER

soziologiemagazin e.V.

Ludwig-Maximilians-Universität München

Institut für Soziologie

Konradstraße 6

80801 München

RECHTSSITZ: Halle (Saale)

VEREINSVORSTAND (VISDPR)

Claas Pollmanns (Vorsitzender)

Andreas Schulz (stellv. Vorsitzender)

Sarah Kaschuba (Finanzen)

Tamara Schwertel

Markus Kohlmeier

vorstand@soziologiemagazin.de

\section{REDAKTION}

Andreas Schulz, Claas Pollmanns, Clément

Dréano, Eva-Maria Bub, Franziska

Deutschmann, Frederic Markus Gerdon, Hendrik Erz, Laura Porak, Markus Kohlmeier, Markus Rudolfi, Sam Schneider, Sarah Kaschuba, Saskia Reise, Tamara Schwertel, Tanja Strukelj, Tatiana Huppertz, Veronika Riedl, Wibke Henriette Liebhart

FRAGEN BITTE AN

redaktion@soziologiemagazin.de

EDITORIAL DESIGN

Veronika Riedl

\section{ILLUSTRATIONEN UND FOTOGRAFIEN}

Veronika Riedl

\section{ANZEIGEN}

Ansprechpartner: Markus Kohlmeier

anzeigen@soziologiemagazin.de

Es gilt die Anzeigenpreisliste vom 01.05.2015

\section{WISSENSCHAFTLICHER BEIRAT}

Prof. Dr. Brigitte Aulenbacher, Prof. Dr. Birgit Blättel-Mink, Prof. Dr. Ulrich Bröckling, Prof. Dr. Aldo Haesler, Prof. Dr. Ernst von Kardorff, Prof. Dr. Hubert Knoblauch, Prof. Dr. em. Reinhard Kreckel, Prof. Dr. Thomas Kron, Dr. Diana Lindner, Prof. Dr. Kurt Mühler, Dr. Yvonne Niekrenz, Dipl. Sozialwirt Harald Ritzau, Dr. Cornelia Schadler, Dr. Imke Schmincke, Dr. Jasmin Siri, Dr. Irene Somm, Prof. Dr. Manfred Stock, Dr. Sylvia Terpe, Prof. Dr. Paula-Irene Villa, Prof. Dr. Georg Vobruba, Dr. Greta Wagner

ERSCHEINEN UND BEZUGSBEDINGUNGEN

Jährlich zwei Hefte. Open Access

PREIS: Einzelheft Print EUR 13,00;

Abonnement Print: EUR 22,00/ Jahr, Abonnement Print ermäßigt EUR 18,00/ Jahr (inkl. MwSt., zzgl. Versandkosten);

E-JOURNAL: kostenlos

Das digitale Angebot finden Sie auf: sozmag.budrich-journals.de und auf www.soziologiemagazin.de

\section{BESTELLUNGEN PRINT}

bitte an den Buchhandel oder den

Verlag Barbara Budrich

Stauffenbergstr. 7

D-51379 Leverkusen-Opladen

Tel.: +49 (0)2171.344.594

Fax: +49 (0)2171.344.693

info@budrich.de

www.budrich-journals.de

www.budrich.de

www.budrich-academic.de

Heft 18, Jg. 10, 2018

(c) 2018 Verlag Barbara Budrich

Opladen | Berlin | Toronto

ISSN 2198-980X 


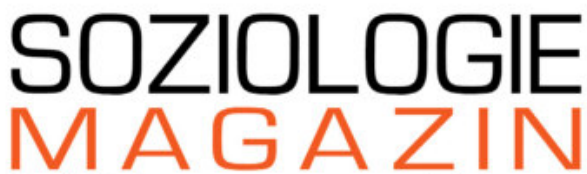

Publizieren statt archivieren

\section{Rausch und Ekstase}

\section{Erkundungen der Spaßgesellschaft}

„Überhaupt aber tragen glänzende, rauschende Feste und Lustbarkeiten stets eine Leere, wohl gar einen Mißton im Innern; schon weil sie dem Elend und der Dürftigkeit unseres Daseins laut widersprechen."

(Arthur Schopenhauer)

Analog zu Schopenhauer werden Rausch und Ekstase auch heute noch oftmals als mehr oder weniger notwendige und legitime Strategien zur Flucht aus der Monotonität des hiesigen Alltagslebens wahrgenommen. Der Bruch mit dem Gewohnten in diesem Zusammenhang ist dementsprechend ein selbstverständlicher Teil unseres Miteinanders. So suchen Erlebnishungrige nach dem Außeralltäglichen und leisten dafür, wie Gerhard Schulze beschreibt, „Erlebnisarbeit“. Dabei kann das ,Dionysische' nach Michel Maffesoli durchaus vergemeinschaftend wirken und somit wichtige soziale Funktionen erfüllen. Und auch darüber hinaus werden Rausch und Ekstase als wichtige Möglichkeiten erachtet, dem rationalen und ökonomischen Imperativ der modernen Gesellschaft zu entgehen. Umgekehrt wird die Lust am Ausbruch im Zuge einer zunehmenden „Eventisierung” zum „marktstrategischen Massenspaß” (Ronald Hitzler) und verkehrt sich dementsprechend selbst zum Imperativ.

In der 19. Ausgabe des Soziologiemagazins begeben wir uns auf die Spuren von Rausch und Ekstase. In diesem Zusammenhang wollen wir wissen:
Wie sieht sie aus, die viel gescholtene Spaßgesellschaft? Gibt es sie überhaupt? Was machen Rausch und Ekstase aus? Wie steht es um die Möglichkeiten der Affizierbarkeit? Wirken Rausch und Ekstase gemeinschaftsbildend oder gar identitätsstiftend? In welchem Zusammenhang stehen Rausch und Ekstase mit den ökonomischen Verhältnissen? Wer berauscht sich wie? Gibt es milieu-, geschlechts- und/oder generationsspezifische Unterschiede? Welche Möglichkeiten bieten Rausch und Ekstase dem rationalen und ökonomischen Imperativ der modernen Gesellschaft zu entgehen oder ist das Gegenteil der Fall? Ist die Lust am Ausbruch per se apolitisch und hedonistisch? Auch wenn im Zustand der Ekstase gesellschaftliche Schranken aufgelöst zu sein scheinen, stellt sich uns die Frage, ob und wenn ja welchen sozialen Regeln Rausch und Ekstase folgen?

Dies und noch viel mehr wollen wir von euch wissen. Sendet Eure Texte zum Thema bis zum 1. Dezember 2018 an einsendungen[at]soziologiemagazin.de.

Natürlich sind wir darüber hinaus immer und themenunabhängig an Rezensionen, Interviews und Tagungsberichten interessiert.

Hilfestellungen für Eure Artikel bekommt Ihr auf unserer Website unter „Hinweise für Autor_innen“. 